\title{
The Dawn of the Mesolithic on the Plains of Poland
}

\author{
Tomasz Płonka $^{1}$ (D) Dariusz Bobak ${ }^{2}$ (D) Michał Szuta $^{1}$
}

Published online: 13 October 2020

(c) The Author(s) 2020

\begin{abstract}
In this article we take a fresh look at the population dynamics of the Polish Plain in the transition from the Pleistocene to the Holocene, using Bayesian analysis and modelling of radiocarbon dates, and contrast the results with data from the North German Plain. We argue against simple adaptationalist models and instead see the cultural landscape as a complex patchwork of old forms and the emerging new traits of the early Mesolithic. We argue that the Mesolithic directly follows the Final Palaeolithic on the Polish Plain, without the chronological hiatus of 150-300 years that is often assumed for that region; while, by contrast, the two cultural patterns-Final Palaeolithic and microlith-based Mesolithic_-overlapped significantly in time on the adjacent North German Plain.
\end{abstract}

Keywords Late Palaeolithic · Early Mesolithic · Radiocarbon dating · Pleistocene/ Holocene transition $\cdot$ Settlement hiatus $\cdot$ Migration

\section{Introduction}

Notwithstanding many years of studies, the mechanism through which hunter-gatherer societies of northern and Central Europe adapted to environmental changes at the transition from the Pleistocene to the Holocene has not yet been satisfactorily described. Clearly, the transition from the Pleistocene to the Holocene is a period of dramatic climate change accompanied by the profound economic and cultural transformation of hunter-gatherer societies worldwide. These changes are particularly visible in the northern portions of America and Europe, where the significant warming of the climate brought about the replacement of tundra by woodlands with

Electronic supplementary material The online version of this article (https://doi.org/10.1007/s 1096 3-020-09146-0) contains supplementary material, which is available to authorized users.

Tomasz Płonka

tomasz.plonka@uwr.edu.pl

1 Institute of Archaeology, University of Wrocław, ul. Szewska 48, 50-362 Wrocław, Poland

2 Institute of Archaeology, University of Rzeszów, ul. Moniuszki 10, 35-015 Rzeszów, Poland 
their associated fauna. In the eyes of many scholars studying this period, the transition from the Palaeolithic to the Mesolithic on the plains of Poland was accompanied by major population changes, as Palaeolithic hunters followed herds of reindeer on their northward migration, thus abandoning the area. Subsequently, and after a hiatus of up to three hundred years, the depopulated plains were thought to have been resettled by hunter-gatherers with Mesolithic cultural traditions. In this paper we challenge this simplistic view and argue that the relationship between the pace of global climatic changes and transformations of society and culture was more complex than usually assumed in simple adaptationist theories.

In focussing on the Final Palaeolithic and early Mesolithic in the central section of the European Plain-in the Polish Plain and the uplands bordering it to the south-we draw the line between the Final Palaeolithic and the Mesolithic not only chronologically, using an approach that takes into consideration climate change, socio-cultural transformation, and genetic data. The use of an inflexible chronological framework - that is, viewing the break between the Pleistocene and Holocene as also constituting the borderline between the Palaeolithic and the Mesolithictends to obscure the various changes in the life of hunter-gatherer communities that occurred at the onset of the Holocene. For both the Final Palaeolithic and the earliest stages of the Mesolithic, the genetic data at our disposal is too sparse to serve as a basis for a hypothesis addressing the differences between those two periods (Brace et al. 2019; Günther et al. 2018; Sánchez-Quinto et al. 2012). Hence this paper will differentiate between the Final Palaeolithic and Mesolithic using cultural data, especially those related to technology.

The term Late Palaeolithic will be used in relation to a period beginning with the exploitation of the European Plains by hunters and gatherers of the Hamburgian, c. 13,600-12,800 cal BC (13,000-12,500 BP). This period covers also later traditions of the Arch-backed Point Complex (Federmesser) and the Tanged Point Complex (Brommian, Ahrensburgian and Swiderian). The term Final Palaeolithic refers to the very end of the Palaeolithic and is used for the sites from c. 10,500 cal $\mathrm{BC}$ and younger, mostly Ahrensburgian and Swiderian ones, continued with the socalled Long Blade Industries or the Epi-Ahrensburgian. The people of those cultures centred their economy on late glacial fauna (mostly reindeer), although at the onset of the Holocene they also resorted to woodland-dwelling animals. Their hunting implements consisted mainly of tanged points and backed pieces (late Federmesser), while their organic-material tools included Lyngby axes and harpoons-whether uniserially or biserially barbed-made from reindeer antler. The large numbers of geometric microliths among excavated weapons, mainly triangles and truncated pieces, and of various points and harpoons made from antlers or bone from deer and other woodland fauna, is characteristic of the Mesolithic. Elk antler mattocks are yet another implement characteristic of the onset of the Mesolithic in NW Europe.

The transition from the Palaeolithic way of life to a life in the Early Holocene woodlands of the plains where Poland lies today has been the subject of lively debate for many years. The underlying cause - a warming post-glacial climate and the related ecosystem response-has always been clear, but the speed of sociocultural and technological changes, and the mechanisms driving them, continue to be open to various interpretations. A few years ago these issues were addressed in 
detail in subject literature by Polish researchers specialising in this area (Kabaciński 2016; Kabaciński and Sobkowiak-Tabaka 2010; Kobusiewicz 1999; Schild 1996a, 2001; Sobkowiak-Tabaka 2011, 2016). The same transition has also been the subject of intense debate in the context of the North German Plain, that is, the westernmost segment of the Central European Plain (the western and northwestern sections of the Plain: Brinch Petersen 2009; Conneller et al. 2016; Crombé et al. 2009; Gramsch 1973; Groß 2014; Møller Hansen and Buck Pedersen 2006; Rozoy 1978; Sørensen et al. 2018; Street et al. 2001; Tolksdorf et al. 2009; Terberger 2004, 2006; Weber et al. 2011; Zander 2016).

Polish scientific literature on the subject traditionally held that the transition from the Palaeolithic to the Mesolithic in Poland was related to large-scale human migrations (Galiński 1997, 2002; Ginter 1973; Kabaciński 2016; Kabaciński and Sobkowiak-Tabaka 2010; Kobusiewicz 1999; Kozłowski 2001; Kozłowski and Kaczanowski 2003; Kozłowski and Kozłowski 1975, 1977; Kozłowski 1969, 1972; Schild 1996a, 1996b, 2001; Sobkowiak-Tabaka 2011, 2016; Więckowska 1975). Such a perspective envisioned that Final Palaeolithic hunters-particularly communities representing the Swiderian-left the Polish plains and neighbouring uplands and moved north in the wake of migrating herds of reindeer. Such a process, according to the chronology developed through radiocarbon dating and palynological data, happened at the onset of the Preboreal. Reindeer hunters were replaced by an inflow of huntergatherers from the West-and possibly South-who represented Mesolithic cultural traditions and were already adapted to a woodland habitat. Many researchers place the arrival of these hunters some 150-300 years after the envisioned exodus of the Final Palaeolithic population (Kabaciński 2016; Kozłowski 2001; Schild 1996a, 1996b; Sobkowiak-Tabaka 2016). Hence a population hiatus was envisaged for the area today occupied by Poland.

In recent years, the growing number of radiocarbon dates and new techniques of sample preparation, along with the use of accelerator mass spectrometry (AMS) and statistical modelling of dates have all contributed to a more robust foundation on which to base discussions of the chronology of change (Blockley et al. 2004; Bronk Ramsey 1995, 2009a, 2009b; Bronk Ramsey et al. 2006, 2010). Attempts at identification of the pace of development of early Mesolithic settlements in other parts of Europe have resulted in interesting findings which provide a mathematical and statistical foundation for chronological hypotheses (Conneller et al. 2016). It is now possible to treat dates provided by radiocarbon analysis as mathematical data with a certain probability distribution. Previously these had been analysed and treated like dates of historical events, and although in the best circumstances their analysis was conducted by the clustering of radiocarbon dates with a single linkage method (Schild 1996b, Figs 3, 4), one should keep in mind that in the 1990s calibration techniques were very unreliable. We intend to use statistical modelling-which allows for more precise definition of time brackets of studied events-for a more detailed description of the phenomena behind those dates. Chronological models produced in this way will serve as the basis for examination of the mechanisms and pace of cultural changes in the Polish Plain at the transition from the Final Palaeolithic to the Mesolithic. We will supplement our efforts with modelling of radiocarbon dates from the Pleistocene-Holocene transition from the North German Plain, in order to 
examine cultural and population changes from a broader perspective. When discussing the social phenomena accompanying such changes, particular attention will be paid to the issue of possible human migrations - such population movements being extensively used in previous interpretations of cultural changes in the Polish Plain during the transition from the Pleistocene to the Holocene. The dates presented in the text are calibrated radiocarbon date estimates given in the standard cal BC form at $95.4 \%$ (2 Sigma) range, with uncalibrated radiocarbon ages given as BP in brackets. The calibration of radiocarbon measurements was made using the programme OxCal, version 4.3 (Bronk Ramsey 2009a; Bronk Ramsey and Lee 2013) with a ${ }^{14} \mathrm{C}$ age calibration curve IntCal 13 (Reimer et al. 2013). The $B C$ dates in italic present the chronology based on the Bayesian modelling of (calibrated) date estimates.

\section{Study Area}

The area covered by this study lies at the junction of two large physico-geographical regions, the North European and East European Plains (Kondracki 1998; Solon et al. 2018). Most of the evaluated material comes from the former, with only a few sites from northeastern Poland (Dudka, Łajty, Morag, Krutynia River) being located in the Masurian Lakeland, a part of the Eastern Baltic-Belarus Lowland and thus included in the East European Plain. The subject area is bordered from the south by uplands and low mountain ranges, the Sudeten and Carpathians, with only a small section of the latter - the Tatra Mountains_exceeding 2000 metres above sea level (masl) The Polish Plain drains to the Baltic through two main river systems- the Vistula and Oder-and through some smaller river systems, for example the Łyna, a tributary of the Pregoła River and the Pomeranian rivers, for example the Rega, Parsęta, and Wieprza, that empty directly into the Baltic.

The relief of the Polish Plain was in large part shaped during Pleistocene glaciations, the latest of which, the Weichselian, at its peak covered northern and northwestern Poland (Fig. 1). A post-glacial landscape consists of a rolling plain with lines of terminal moraines as well as other features associated with glaciations: outwash plains, drumlins and eskers. Some moraines exceed 300 masl; Wieżyca, the highest point of the North European Plain, has an absolute height of 328.7 masl, and stands some $270 \mathrm{~m}$ above the surrounding terrain. At the end of the Pleistocene the Polish Plain was subject to extensive aeolic accumulation processes, in some places forming entire dune fields (Kozarski et al. 1969; Nowaczyk 1986; Zeeberg 1998). One of the largest such fields is found in western Poland, between the Warta and Noteć Rivers, with some of the dunes having a relative height of 20-40 m (Pilarczyk 1958, 1976). The underlying tectonic structure, with an inclination towards the northwest, produces asymmetric river basins, this being particularly visible in the case of the major rivers running south to north, namely the Vistula and Oder, whose right-bank tributaries significantly outnumber left-bank ones. The areas covered by the latest glaciations contain many lakes of different size (up to $113.8 \mathrm{~km}^{2}$ ) and 


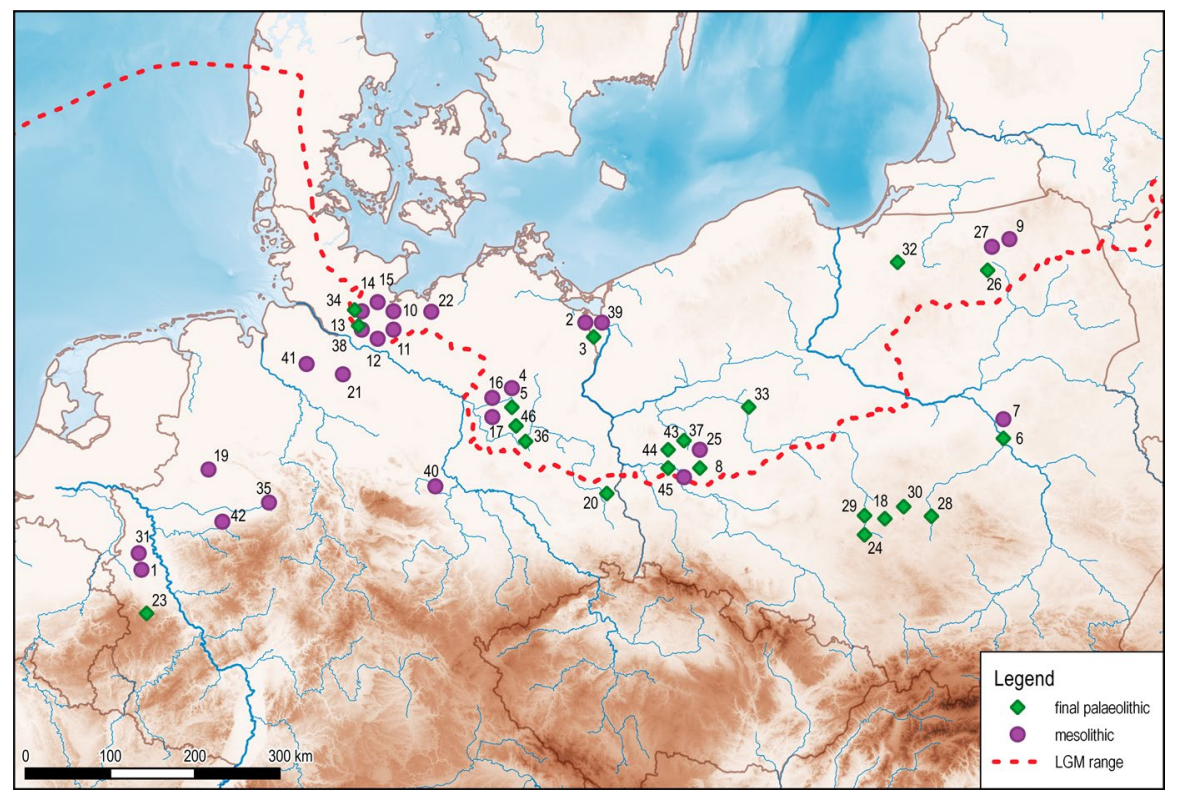

Fig. 1 Final Palaeolithic and early Mesolithic sites with radiocarbon dates in the North German and Polish Plain, and the Eifel. 1-Bedburg-Königshoven 2, 3-Bolków 1; 4, 5-Bützsee-Altfriesack; 6, 7-Całowanie 3; 8-Chwalim 1; 9-Dudka 1; 10-Duvensee 1; 11-Duvensee 11; 12-Duvensee 2; 13-Duvensee 6; 14-Duvensee 8; 15-Duvensee 9; 16-Friesack 27a; 17-Friesack 4; 18-Grabek 11; 19-Greven-Schencking sandpit; 20-Groß Lieskow; 21-Haverbeck; 22-Hohen Viecheln; 23Kartstein; 24-Kochlew 1; 25-Kopanica 29; 26-Krutynia river; 27-Łajty 1; 28—Łęg Ręczyński; 29-Łykowe 1; 30-Mokracz; 31-Mönchengladbach-Geneicken; 32-Morąg; 33-Murowana Goślina; 34-Nahe LA 11; 35-Paderborn-Sande; 36-Potsdam-Schlaatz; 37-Smolno Wielkie 2; 38-Stellmoor; 39-Tanowo 3; 40-Unseburg; 41-Wehldorf 7; 42-Werl-Büderich; 43-Wojnowo 2; 44Wojnowo 4; 45-Wojnowo a; 46-Wustermark 22

depth (up to $106 \mathrm{~m}$ ), which in many epochs have attracted human settlement to their banks.

The North European Plain is part of the lowlands running longitudinally from the Urals to the Atlantic. It has no real natural borders setting it off from the lowlands to east or west. For instance, the Oder River, used as the western limit of the Polish Plain, is not much of a natural barrier. The German sites included in this study are mostly located on the North European Plain, the exception being Kartstein in the Eifel. There are no major natural obstacles to the migration of fauna and flora, or to the westward movement of air masses-maritime, from the Atlantic, and inland, or continental, from Russia. Neither does the lay of the land hinder the movement of human groups, from West to East or South to North. At the close of the Pleistocene the mountain ranges to the south of the Polish Plain, the Sudeten and Carpathians, did not constitute any sort of impassable barrier, as a result both of their unimpressive elevation and of numerous passes and gaps 
(Burdukiewicz 1999, p. 49; Burdukiewicz and Bronowiecki 1999, pp. 176-179; Rydlewski and Valde-Vowak 1979, pp. 5-6, 31-32).

\section{Cultural Background}

We shall not discuss in detail the cultural landscape of Central Europe in 10,500-9000 cal BC, but only provide a short summary of the cultural traditions then in existence. At the end of the Pleistocene, this area was inhabited by huntergatherer communities of the Tanged Point Complex, whose way of life was largely dependent upon reindeer herds. Two cultures are identified, the Ahrensburgian in the West and the Swiderian in the East, and these differ in the types of tanged points used-Ahrensburgian or Swiderian, the latter with a flat retouch of a tang or a base on a ventral side. Such tools were made from blades knapped off mainly from double platform cores with opposite platforms. A few researchers admit the possible existence of a distinct Ahrensburgian-Swiderian cultural unit in what is now western Poland, which used the two types of tanged points (i.e. both the Ahrensburgian and Swiderian) in parallel (Kobusiewicz 2004). It is also probable that in the Final Palaeolithic there were some groups of the Federmesser tradition in the North European Plain (Sobkowiak-Tabaka 2011).

Current thought holds that the end of the Pleistocene in the North European Plain brought about the appearance of human groups with a modified toolset, termed EpiAhrensburgian, whereas in northern France and southern England we now note characteristic inventories with long blades (Long Blade Industries) knapped from double platform cores with opposite platforms (Barton 1998; Brinch Petersen 2009; Galiński 2018; Gob 1991; Johansen and Stapert 2000; Sørensen et al. 2018; Weber et al. 2011; Zander 2016). Epi-Ahrensburgian inventories from the Netherlands, northern Germany, Denmark, and Sweden as well as northwestern Poland all share the feature of including microlithic forms, mostly small Zonhoven points, as well as no- or very few-tanged points. These late finds are not dated in a reliable manner, with only northern French assemblages - termed Belloisien - and those from southern England being radiocarbon dated to 9700-9400 cal BC; the finds from northwestern Poland are dated to 9600-9300 cal BC, or maybe even later. Such dates give rise to the theory that at the onset of the Preboreal various areas of the European Plain were home to cultural traditions rooted in the Final Palaeolithic.

The beginnings of the Mesolithic in the Polish Plains are linked with the appearance in archaeological inventories of numerous microliths in the form of truncated and backed points, triangles and crescents. These forms dominate toolkits and are chiefly used as arrowhead elements, with bows gaining in popularity in the Holocene woodland environment (Rozoy 1989). Such assemblages from southern Scandinavia are termed the Maglemosian, with the name Duvensee complex applied to North European Plain finds; whereas in Poland the accepted term is the DuvenseeKomornica Complex or the Komornician (Brinch Petersen 2009; Kabaciński 2016; Kobusiewicz 1999; Kozłowski 1989; Terberger 2004, 2006). Today the oldest Maglemosian finds are dated to 9600-9500 cal BC (Jessen et al. 2015), that is, to the 
very dawn of the Preboreal, although other early Mesolithic sites from the North European Plain are slightly younger.

\section{Materials and Methods}

Studies of the chronology of the Final Palaeolithic and early Mesolithic in the North European Plain are affected by the specific conditions influencing deposition. The last global-scale sedimentation event in this area had been the formation of dunes, the last phase of which took place in the Younger Dryas. However, the settlement processes pertinent to this study are of a younger age and occurred later. Hence most Final Palaeolithic and Mesolithic sites dating from the Pleistocene-Holocene transition are found in sandy sediment, in the same level. Such conditions are not favourable to the preservation of organic matter that could be radiocarbon dated. Sites with clear stratigraphical relationships and rich in organic finds are few and far between, and mostly come from the banks of bodies of water subjected to eutrophication during the Holocene. Layers with organic remains allowing environmental studies and radiocarbon dating are usually located at a camp's periphery, at the shoreline of the body of water alongside which the camp was established, or in shallow water.

Other important sources are single finds of bone and antler artefacts of forms typical for the Final Palaeolithic and early Mesolithic. Their dating provides important pointers as to the duration-or point of emergence-of artefacts characteristic of the Final Palaeolithic and early Mesolithic, such as Lyngby axes or specific types of harpoons. Nevertheless, one should keep in mind that such single finds need to be approached with caution, as some forms may have been in existence for longer periods, from the Late Palaeolithic up to the early Mesolithic (Cziesla and Pettitt 2003).

Unworked reindeer antlers may serve as supplementary material for dating purposes. The presence of such antlers implies the existence of a local population of reindeer-a species highly characteristic of the Pleistocene in the Polish Plain. Whereas artefacts made from reindeer antlers might have been imported from the north, it is very unlikely that unworked antlers had been subject to such exchange. Hence the latest finds of such unworked antlers mark the end of the last herds of reindeer in this part of Europe. Naturally this does not preclude imports of antlers or antler artefacts during later phases of the Mesolithic, a good example of which is the recent find of the baton at Gołębiewo, central Poland (Osipowicz et al. 2017).

Our analysis is based upon radiocarbon dating from Final Palaeolithic and Mesolithic sites from the plains of Poland. The dataset consists of dates in the bracket 10,550-8250 cal BC (10,500-9000 BP) (Tables 1, 2, 3, 4). The southernmost sites included in the analysis are Łykowe and Kochlew from the Pyszna and Nieciecz interfluve - an area where the southern Polish Plain abuts the Wielun upland (Majchrowska and Popińska 2018, Fig. 4). As already mentioned, this study also uses-as auxiliary material—radiocarbon dates from this period from sites located in present-day Germany, mostly from the North German Plain, with some dates from the Eiffel (Kartstein) as well (Tables 5, 6, 7, 8). We should point out that some dates from the relevant time brackets were excluded from the analysis: those from stratified sites which are evidently inconsistent with the surrounding stratigraphy 


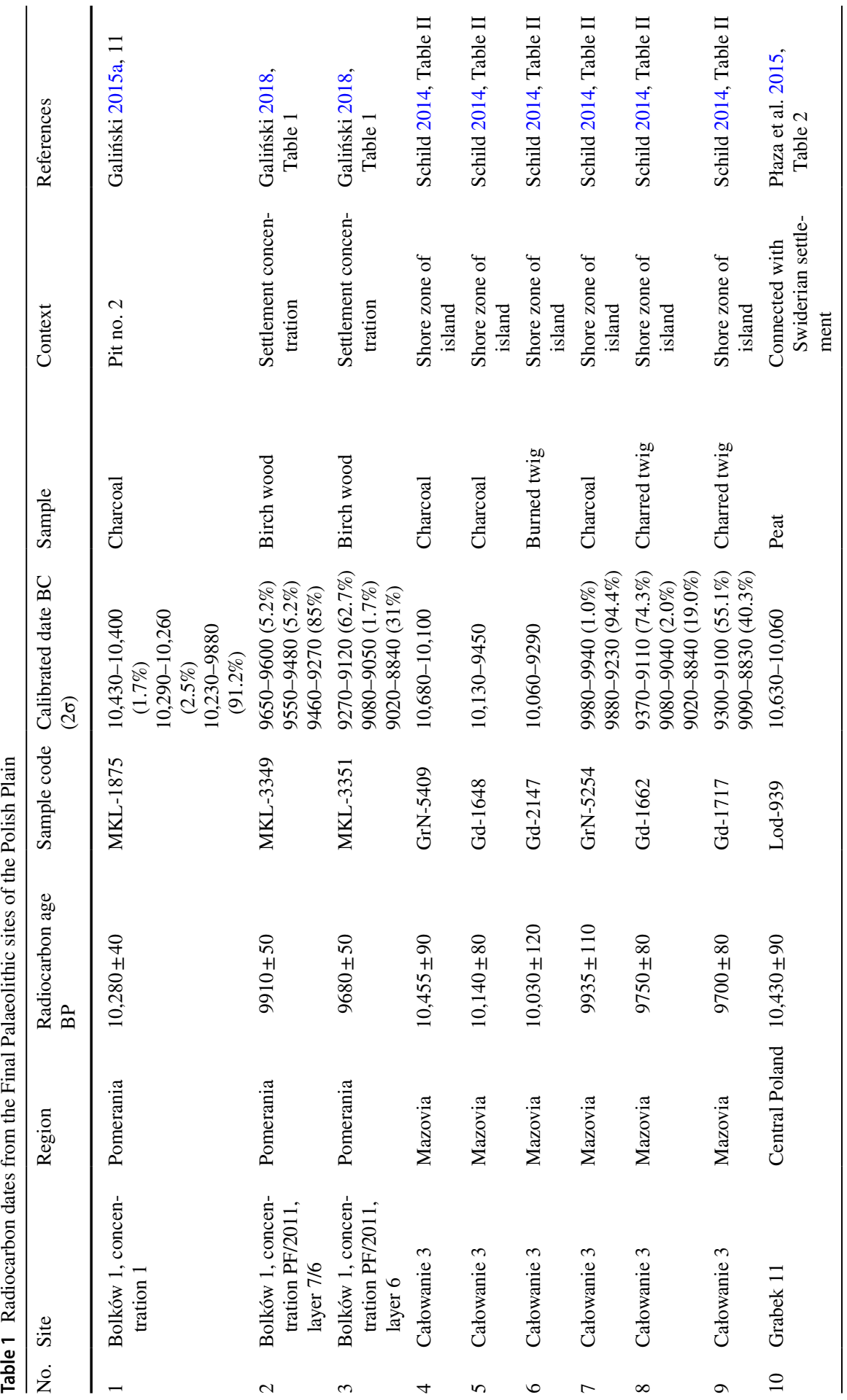




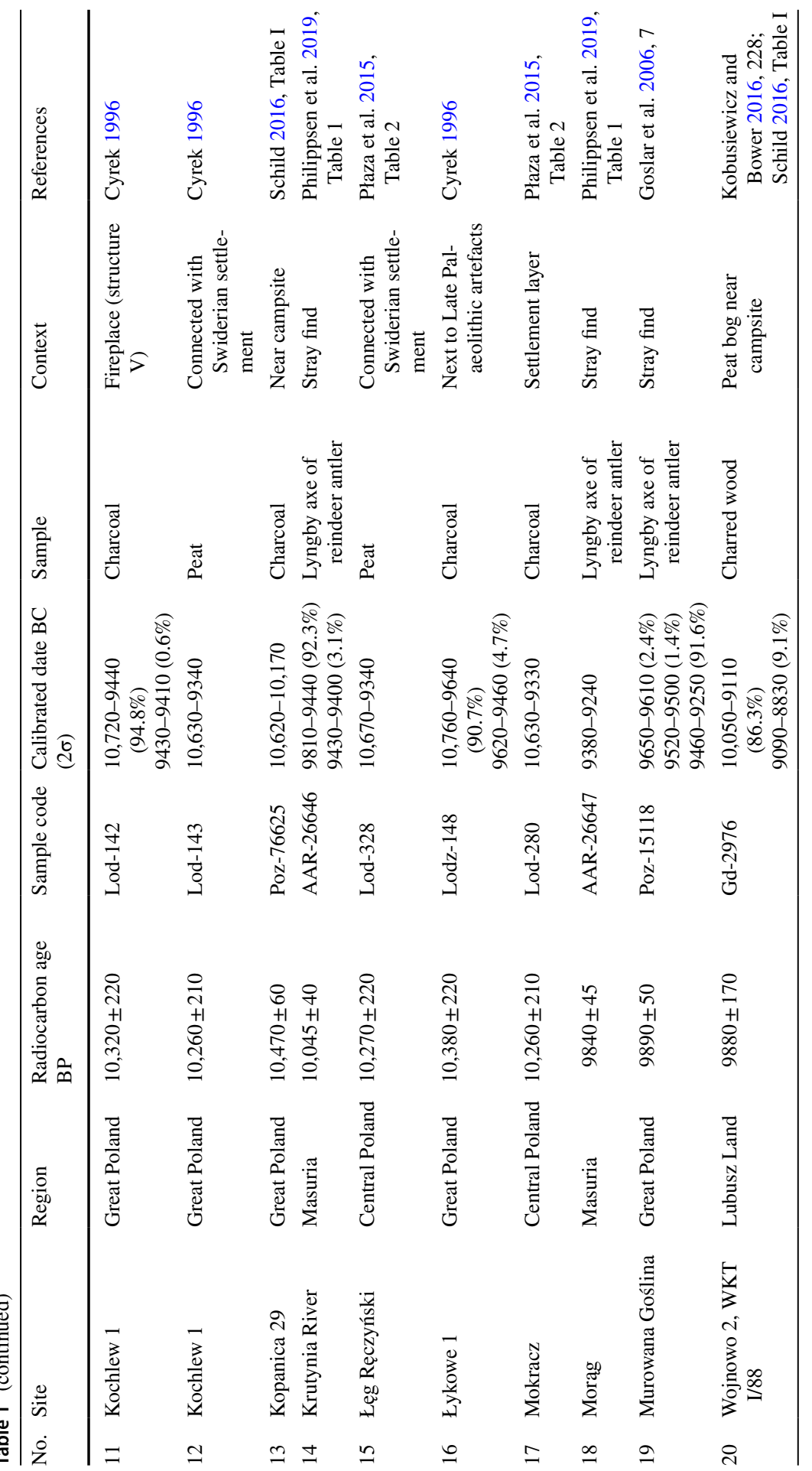




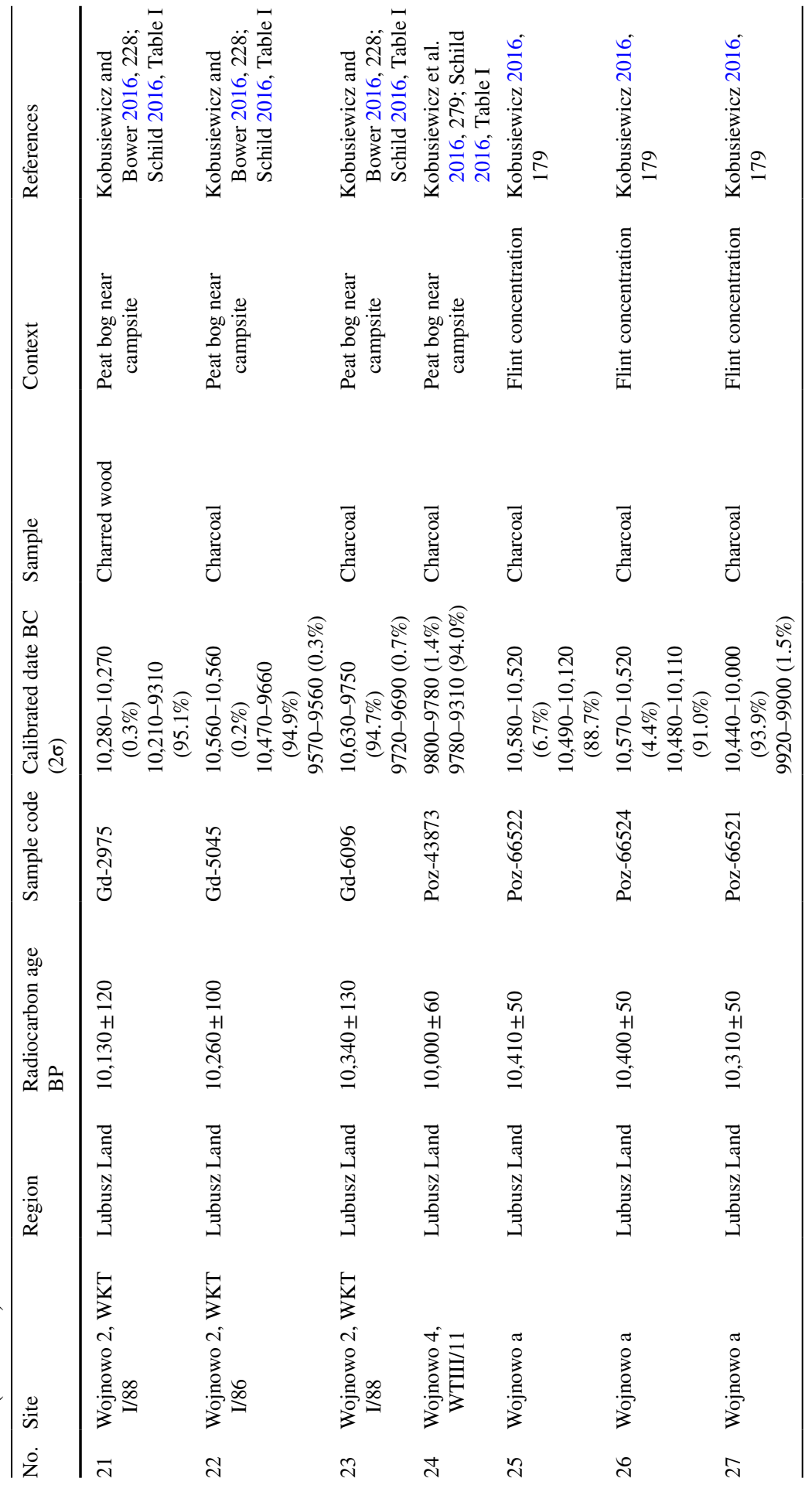




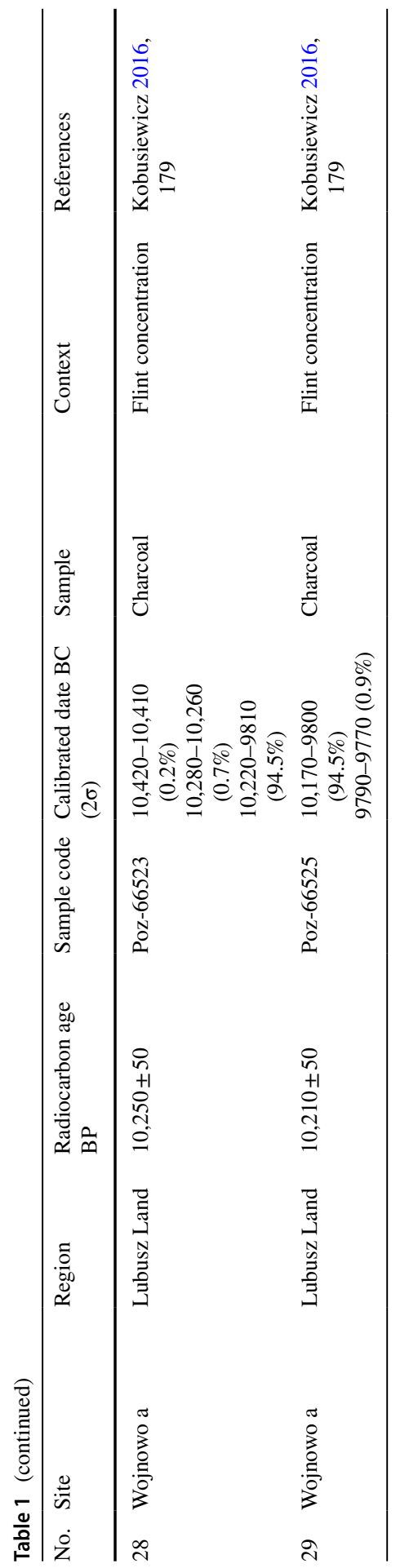


Table 2 Methods of radiocarbon analysis of samples from the Final Palaeolithic sites in the Polish Plain

\begin{tabular}{|l|c|c|c|}
\hline \multicolumn{1}{|c|}{ Dates } & $\begin{array}{l}\text { Radiocarbon dates } \\
\text { without AMS }\end{array}$ & $\begin{array}{l}\text { AMS radiocarbon } \\
\text { dates }\end{array}$ & Number \\
\hline Stratified sites & 15 & 2 & 17 \\
\hline $\begin{array}{l}\text { Flint } \\
\text { concentrations } \\
\text { in sandy areas }\end{array}$ & 4 & 5 & 9 \\
\hline $\begin{array}{l}\text { Stray } \\
\text { antler/bone } \\
\text { artefacts }\end{array}$ & - & 3 & 3 \\
\hline Number & 19 & 10 & 29 \\
\hline
\end{tabular}

and are either too old or too young to be in sequence with the layers they were found in. This is the express reason for rejecting the date from Level $\mathrm{V}$ of the site at Całowanie (Gd-5202). Also excluded are dates going beyond 8250 cal BC from layers termed Sedimentkomplex III at Friesack 4, as these are evidently not related with the pioneer stage of settlement, but rather with an already well-established population (Gramsch 2016, Table 1).

The dataset used for our analysis consists of 160 radiocarbon dates (Tables 1, 2, $3,4,5,6,7,8)$, of which 55 come from Poland and 105 from Germany. Our research identified 29 Final Palaeolithic dates from Poland, originating from 14 sites spread across the Polish Plain (Fig. 1; Tables 1, 2). Twenty-six dates were produced by seven early Mesolithic sites (Fig. 2; Tables 3, 4). Of greatest value for the study are dates from stratified sites, where the samples were taken from clearly defined layers containing settlement structures in the form of flint artefact concentrations. The most relevant finds are those from the site of Całowanie (on an ancient island in the valley of the Vistula) where the site contains stratigraphically arranged remains of Late and Final Palaeolithic, and Mesolithic settlement. Radiocarbon dating of finds from shoreline layers is just as valuable. Of greatest importance are the artefacts from Bolków, site 1, in northwestern Poland (Pomerania), where the habitation from both relevant periods was recorded. A smaller number of dates comes from charcoal and bones found inside flint artefact concentrations in sandy areas, with only three dates coming from reindeer antler items-Lyngby axes. Ten AMS dates (over one-third of the total) are currently available for the Final Palaeolithic, with only five Mesolithic dates derived in this manner. Other radiocarbon dates were produced using older laboratory protocols and their standard deviations are quite significantmost of them in the range 80-320 radiocarbon years; age ranges based upon these dates are consequently quite broad. Note that the attribution of the two oldest dates from Dudka 1 (Nos. 19 and 20 in Table 3) is open to debate; as these contain few flint artefacts the question is - are these dates related to layers associated with early Mesolithic or Final Palaeolithic settlement (Fiedorczuk 1995; Gumiński 1995)? We followed the opinion of Witold Gumiński and consider that it is more likely that the above finds are associated with the early Mesolithic.

Radiocarbon dating from Poland is analysed alongside results of analysis from Final Palaeolithic and Mesolithic sites from northern Germany (Tables 5, 6, 7, 8). Our article takes into consideration 31 Final Palaeolithic dates from seven sites, and 


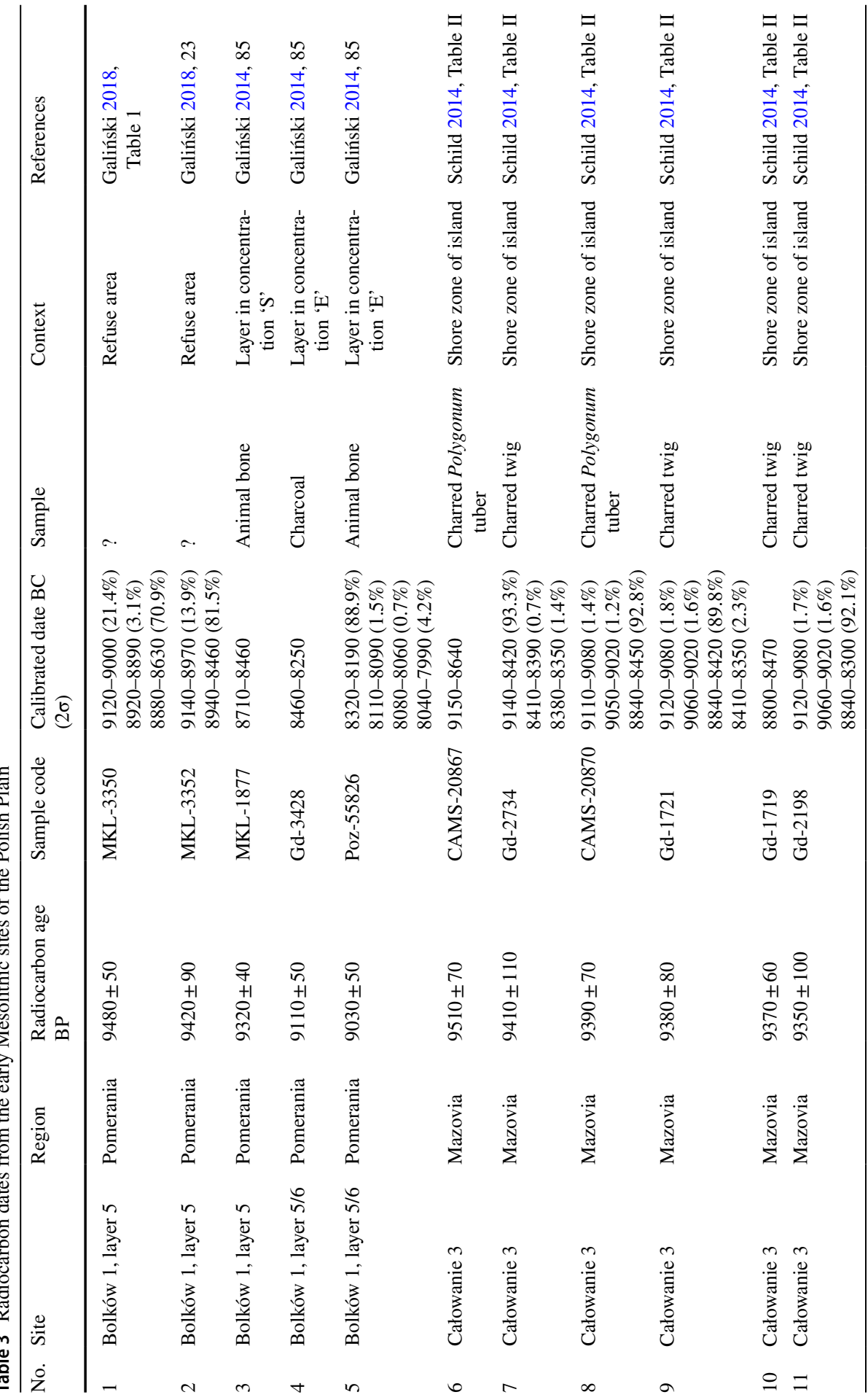




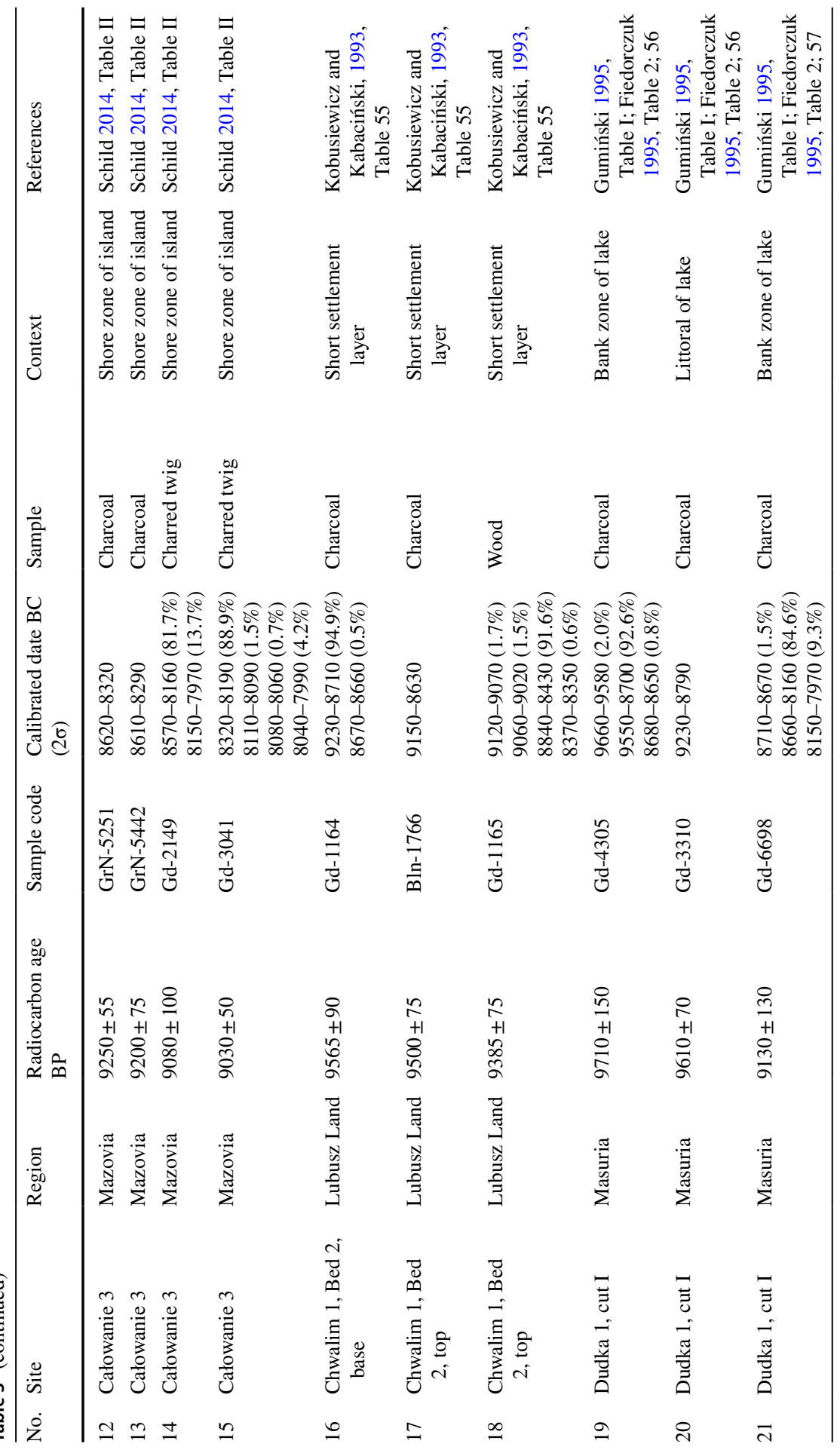




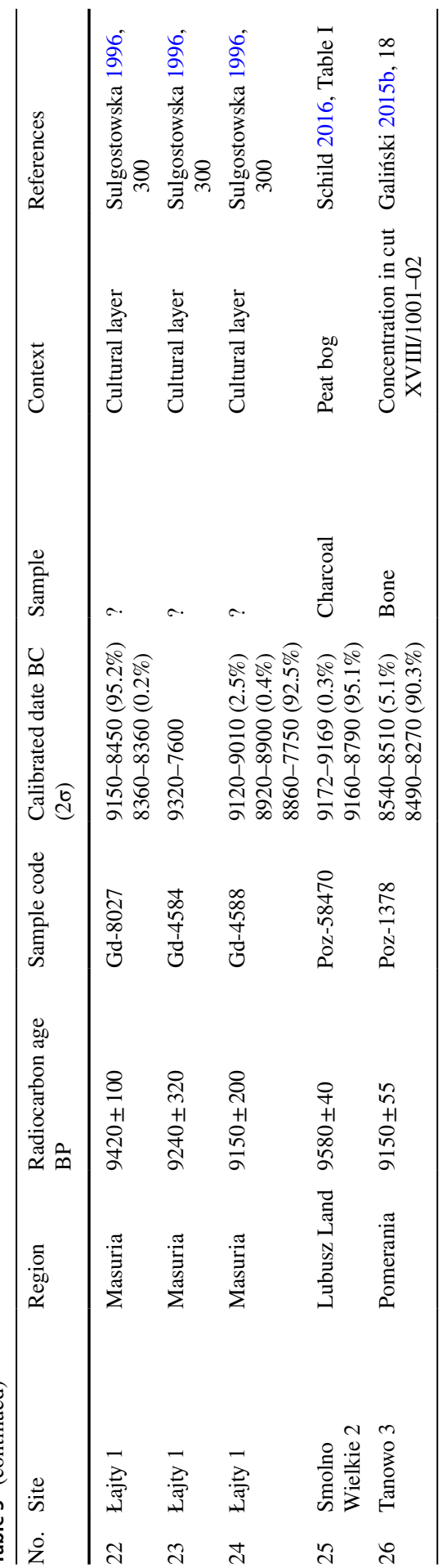


Table 4 Methods of radiocarbon analysis of samples from the early Mesolithic sites in the Polish Plain

\begin{tabular}{|l|c|c|c|}
\hline \multicolumn{1}{|c|}{ Dates } & $\begin{array}{l}\text { Radiocarbon dates } \\
\text { without AMS }\end{array}$ & $\begin{array}{l}\text { AMS radiocarbon } \\
\text { dates }\end{array}$ & Number \\
\hline Stratified sites & 21 & 4 & 25 \\
\hline $\begin{array}{l}\text { Flint } \\
\text { concentrations } \\
\text { in sandy areas }\end{array}$ & - & 1 & 1 \\
\hline $\begin{array}{l}\text { Stray } \\
\text { antler/bone } \\
\text { artefacts }\end{array}$ & - & - & - \\
\hline Number & 21 & 5 & 26 \\
\hline
\end{tabular}

74 dates from 17 early Mesolithic sites. As concerns the Final Palaeolithic dates, a major portion-20, or 64.5\% — comes from Kartstein and Stellmoor. Most of these radiocarbon dates (with the exception of two from Kartstein) were calculated in the 1980s, at laboratories in Köln and Copenhagen, without AMS, with standard deviation up to 45-105 years. Their results are being questioned due to their wide spread, which suggests a very prolonged final phase of the Ahrensburgian, extending into the early Holocene (Street et al. 2001; Weber et al. 2011), which is inconsistent with the model of rapid cultural changes in the early Holocene brought about by rapid climatic and environmental changes. Hence only Younger Dryas dates from these sites are accurate (Weber et al. 2011, pp. 291-292). One should note, however, that no scientific criticism of these dates has previously been made. At the same time, the two dates obtained by the Oxford ORAU laboratory for bones from Stellmoor are from the early Holocene. It is worth adding that the late-i.e. early Holocene-dates for Kartstein were obtained independently at different laboratories_-at Copenhagen and Köln. To accommodate the reservations mentioned above, we used dates from these two sites but we ran two modelling variants-one including Kartstein and Stellmoor dates, and the other without. The radiocarbon date for the butchery site at Potsdam-Schlaatz was usually quoted in the context of early Mesolithic settlement, even though the flint artefacts found alongside an aurochs skeleton were regarded as not representative and thus not useful in identifying the culture of the hunters who had brought down this animal (Gramsch 1987). Nevertheless, blades from this context (Gramsch 1987, Fig. 1: 2-4, 6) bear evident characteristics of Final Palaeolithic flint working: significant length and breadth plus negatives of double platform flaking on the upper side. This led us to assign this site to the category of Final Palaeolithic settlements. Other dates come from settlement horizons of archaeological sites, from refuse layers on a camp's periphery (Wustermark), and from-typical for this period-Havel harpoons.

Of the 74 Mesolithic dates, 38 radiocarbon dates come from the refuse layers of early Mesolithic camps at Friesack (sites no. 4 and 27a) and Hohen Viecheln; 16 come from remains associated with settlement along an ancient lake shoreline near Duvensee (Schleswig-Holstein); and seven come from settlement layers of the Bedburg-Königshaven site. Three dates were obtained from the skeleton of a 50-55-year-old woman from a grave at Unseburg; one date comes from a killing site. Other radiocarbon dates (9) come from charcoal from settlement structures 


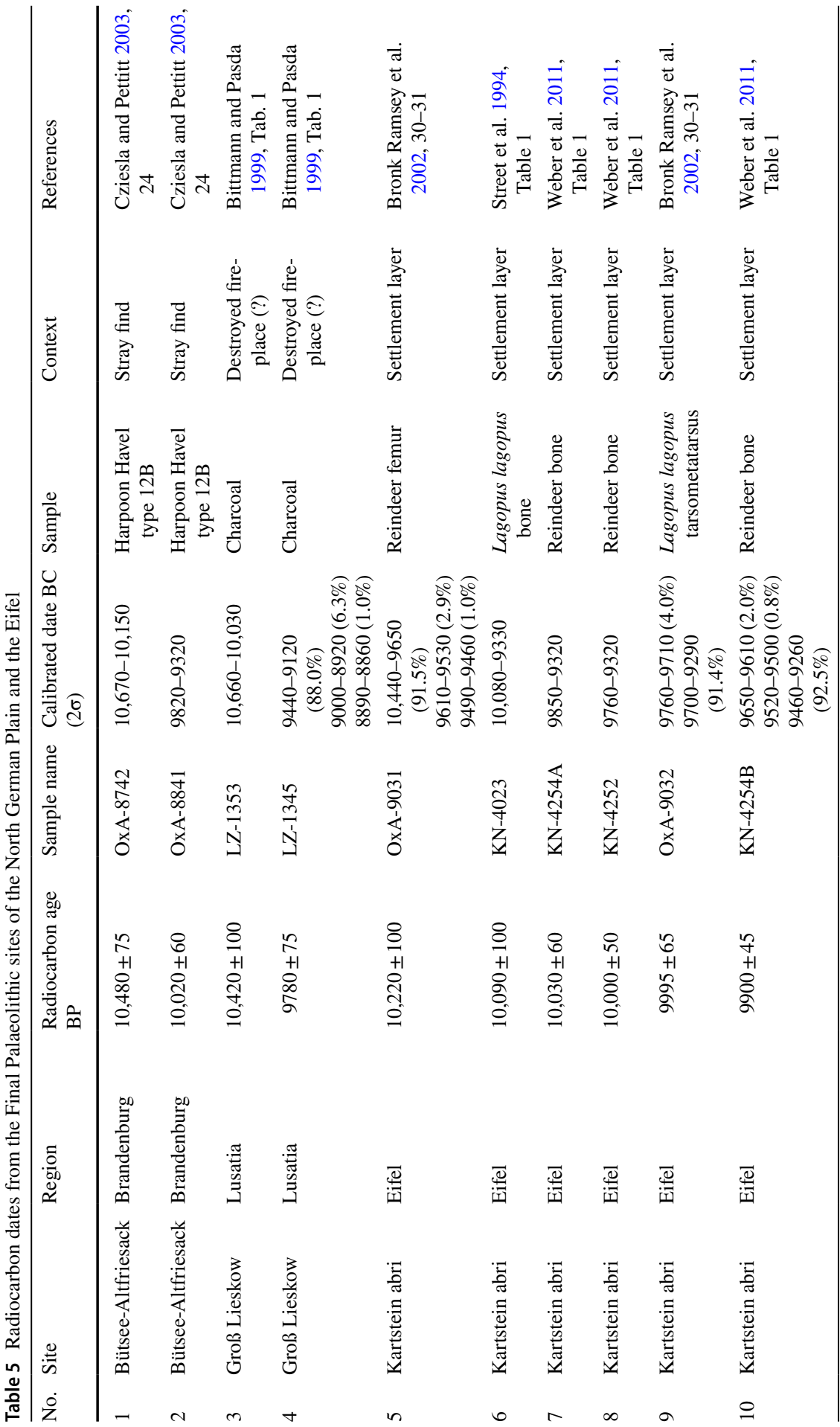




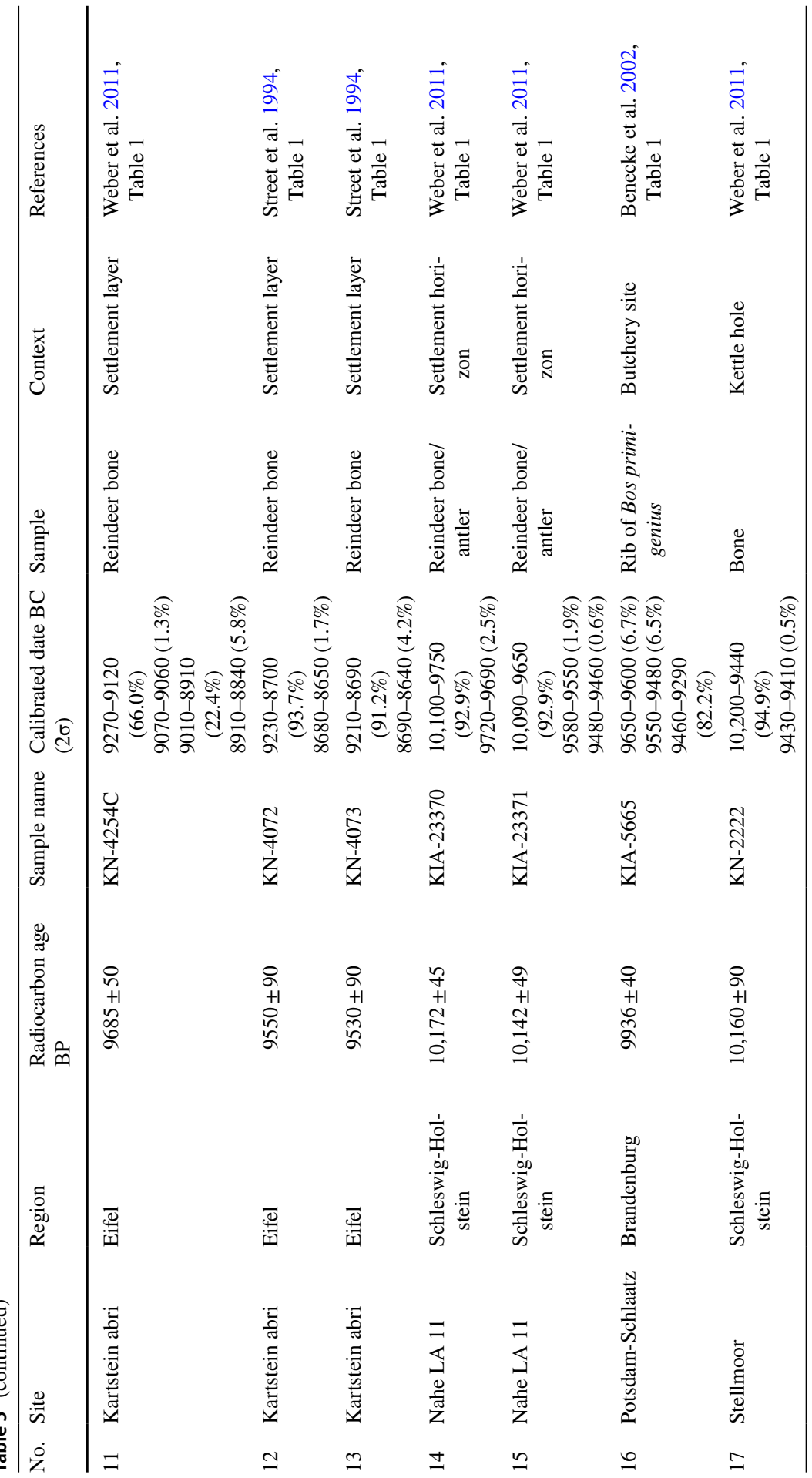




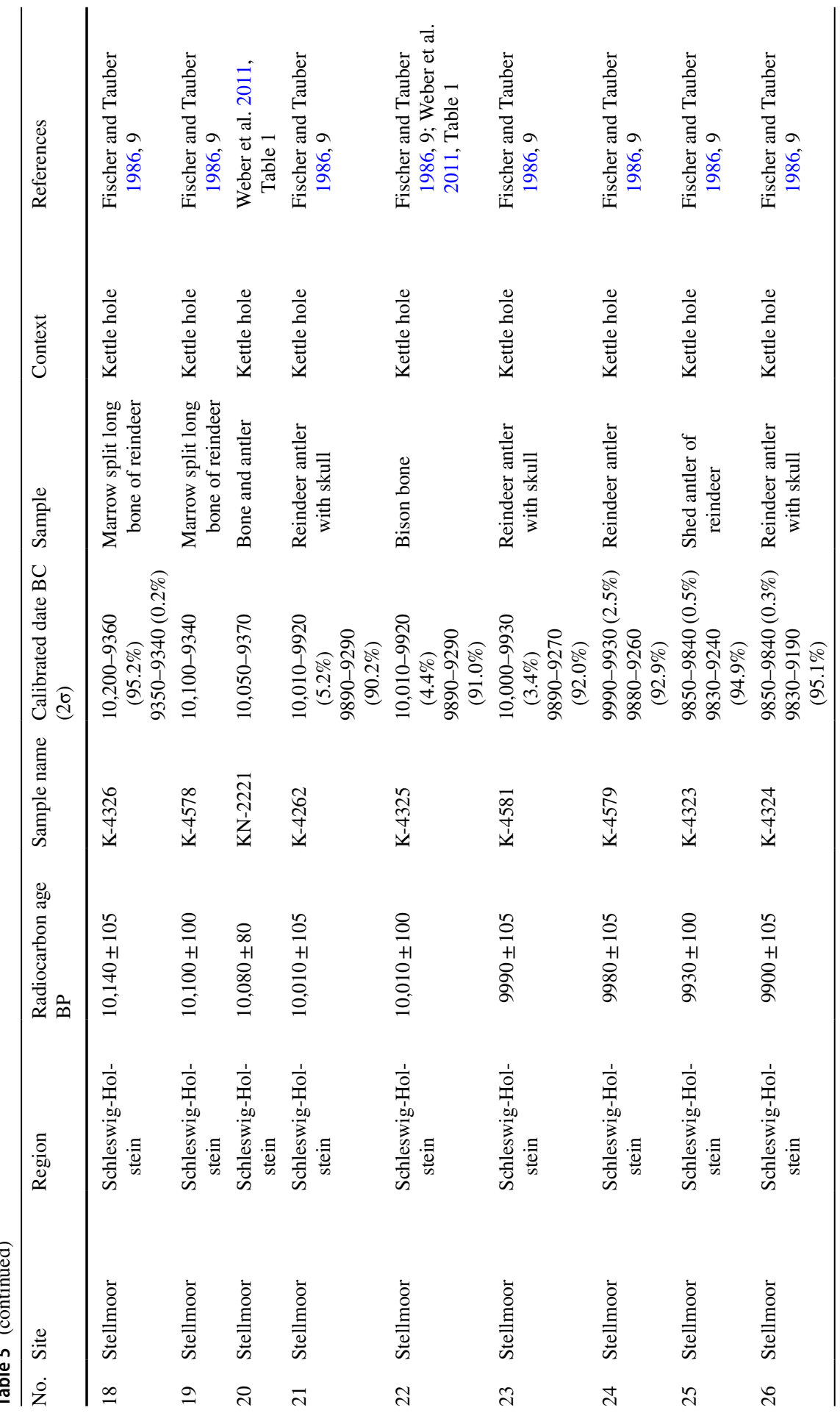




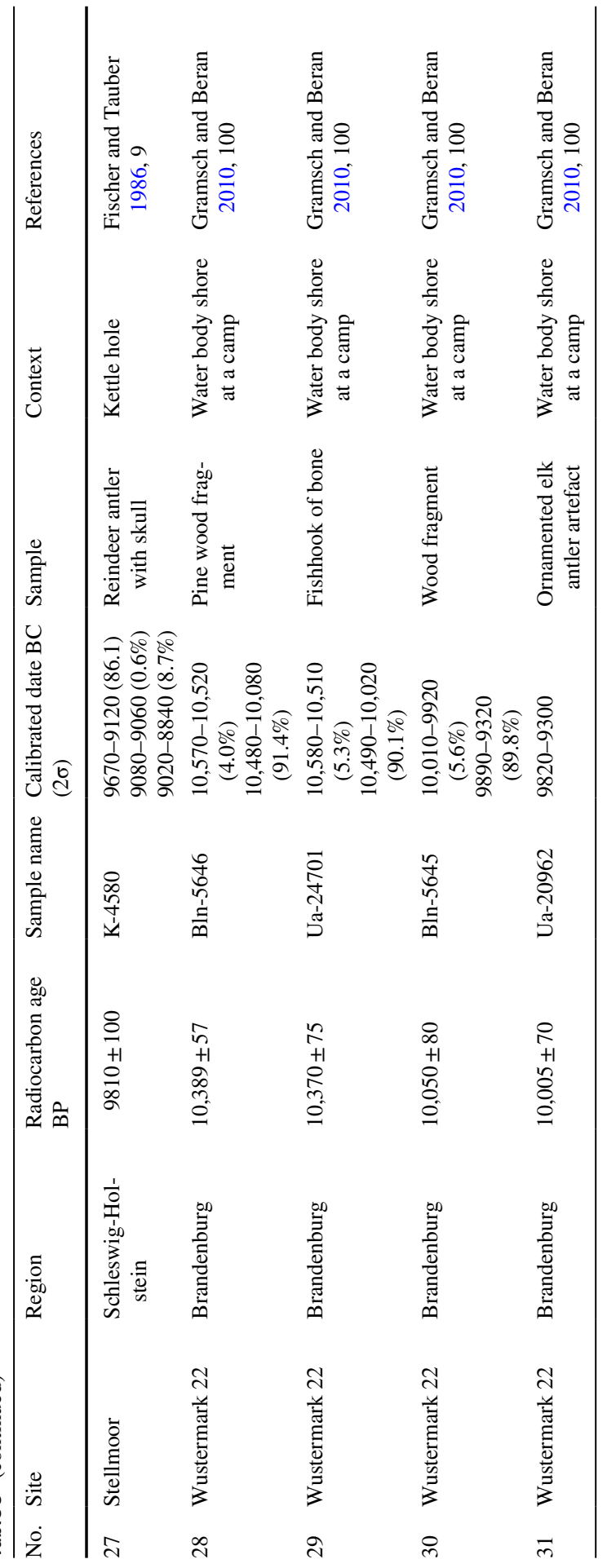


Table 6 Methods of radiocarbon analysis of samples from the Final Palaeolithic sites from the North German Plain and the Eifel

\begin{tabular}{|l|c|c|c|}
\hline \multicolumn{1}{|c|}{ Dates } & $\begin{array}{l}\text { Radiocarbon dates } \\
\text { without AMS }\end{array}$ & $\begin{array}{l}\text { AMS radiocarbon } \\
\text { dates }\end{array}$ & Number \\
\hline Stratified sites & 20 & 6 & 26 \\
\hline $\begin{array}{l}\text { Flint } \\
\text { concentrations } \\
\text { in sandy areas }\end{array}$ & 2 & - & 2 \\
\hline $\begin{array}{l}\text { Stray } \\
\text { antler/bone } \\
\text { artefacts }\end{array}$ & - & 2 & 2 \\
\hline Butchery site & - & 1 & 1 \\
\hline Number & 22 & 9 & 31 \\
\hline
\end{tabular}

and from stray finds of Mesolithic tools: points, harpoons and an elk antler mattock. Only 30 dates were obtained using AMS; the remainder were obtained through traditional methods, with standard deviation being 60-170 years. The oldest dates from Bedburg-Königshaven are questioned as they would suggest that the early Mesolithic pre-dated the Holocene warming. On the other hand their large standard deviation (100 years) places them in a broad time span. Two dates from Duvensee 1 (Bokelmann et al. 1982, Table $1-\mathrm{H} 23 / 22, \mathrm{H} 26 / 23$ ) are from the early days of the Heidelberg laboratory's existence: these dates come with large standard deviations and are usually omitted from tables of dates for the Mesolithic (Weninger et al. 2009, Table 9-6).

A major issue in pinpointing borders of developments during the Pleistocene-Holocene transition is the radiocarbon plateaux found in that period, brought about by a sharp drop in the production of the ${ }^{14} \mathrm{C}$ isotope in the atmosphere as a result of various terrestrial and cosmic phenomena (Kitagawa and van der Plicht 1998). In this period, radiocarbon years are shorter than calendar years. In the relevant timespan, 10,550-8250 cal BC (10,500-9000 BP), the strongest noted plateaux are those for c. 10,350-10,100 cal BC (10,400-10,300 BP), 9550-9350 cal BC (10,000-9900 BP), and 9000-8850 cal BC (9600-9500 BP) (Björck et al. 1998; Bos et al. 2007, p. 1928). For the entire length of the Younger Dryas and early Holocene the difference between radiocarbon years and calendar years may be as much as 1500 years, that is, $10,800-9800$ radiocarbon years BP versus $10,800-9250$ calendar years cal BC (Kitagawa and van der Plicht 1998, p. 1188). Analysis of the chronology of phenomena during the Pleistocene-Holocene transition becomes more reliable, however, when we use the whole dataset of radiocarbon dating and employ Bayesian statistical procedures, which allow for more objective conclusions, independent of climatostratigraphical data (Lowe et al. 2008).

The analysis of absolute dates was performed using a series of Bayesian models made with OxCal 4.3 software (Bronk Ramsey 2009a), with some analysis also using R v. 3.5.3 software (R Core Team 2019), with a ${ }^{14} \mathrm{C}$ age calibration curve IntCal 13 (Reimer et al. 2013).

The fundamental question of the research was the issue of the chronological relations between Final Palaeolithic and Mesolithic settlement in the Polish Plain. 


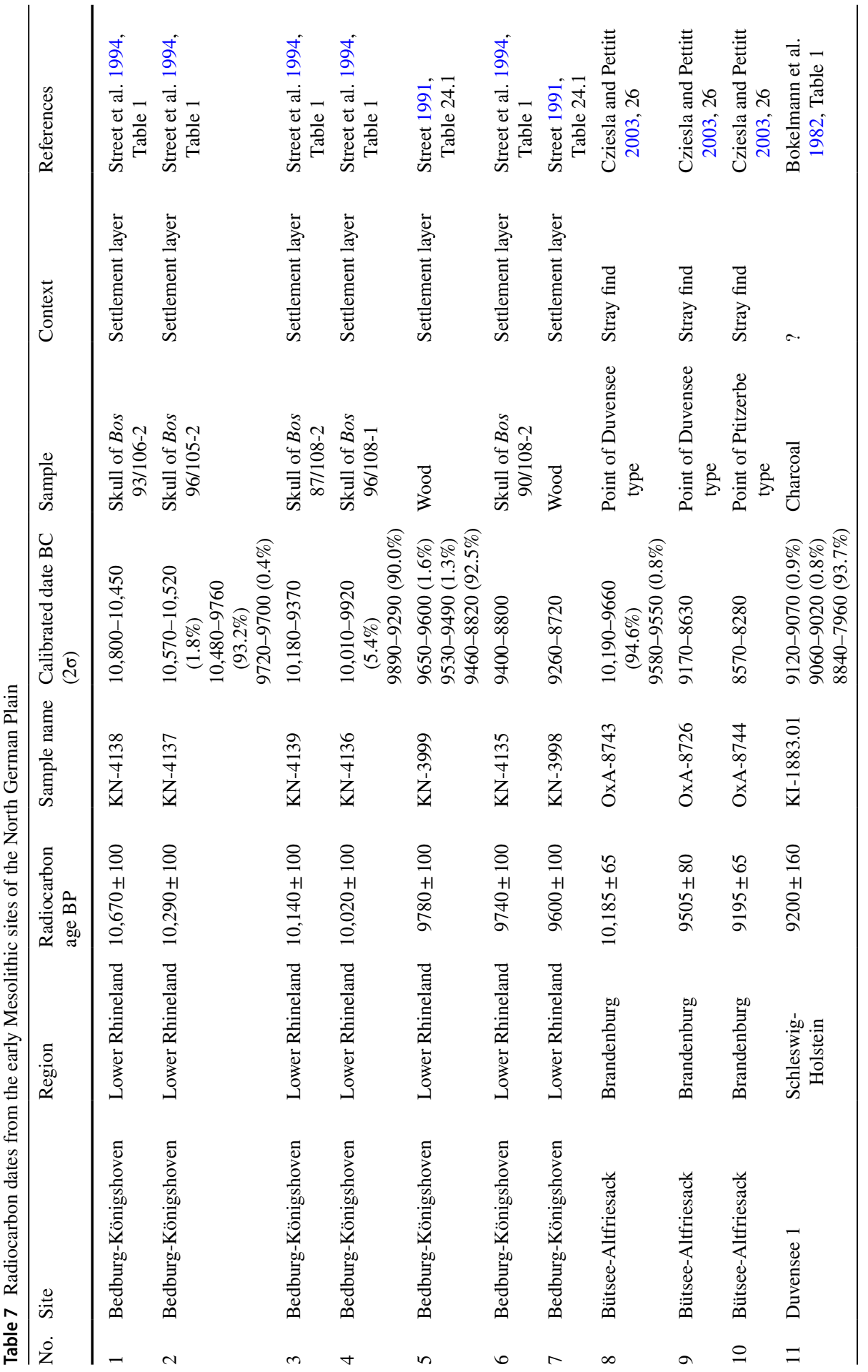




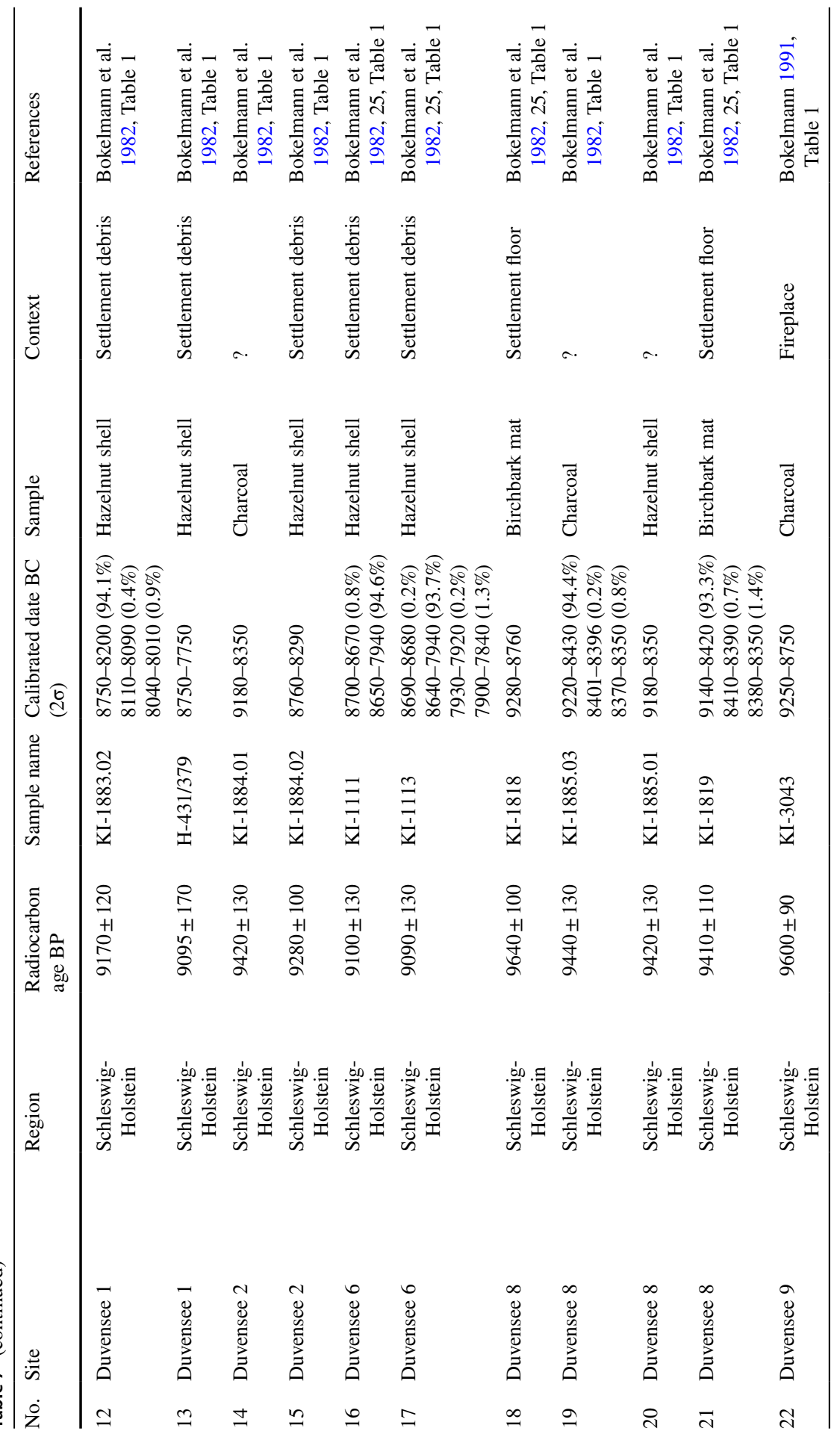




\begin{tabular}{|c|c|c|c|c|c|c|c|c|c|}
\hline 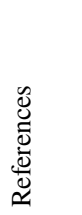 & 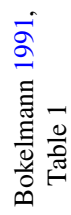 & 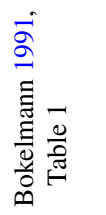 & 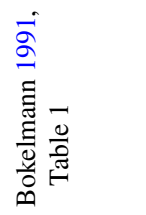 & 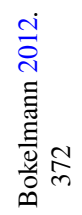 & 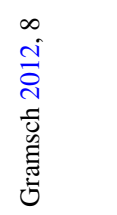 & 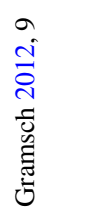 & 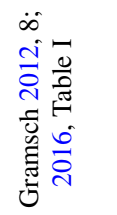 & 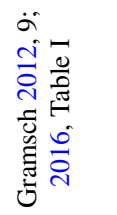 & 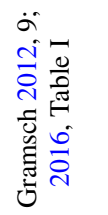 \\
\hline 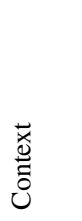 & 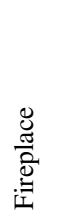 & 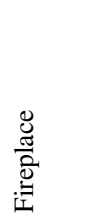 & $\frac{\ddot{g}}{\tilde{0}}$ & 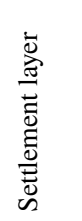 & 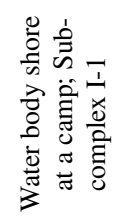 & 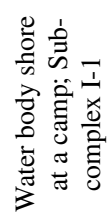 & 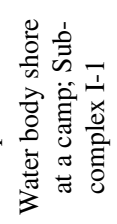 & 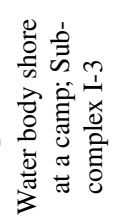 & 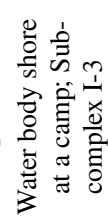 \\
\hline 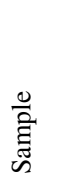 & 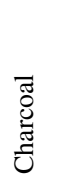 & 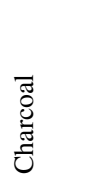 & 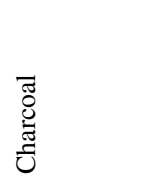 & 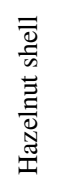 & 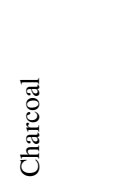 & 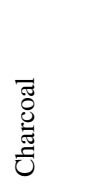 & 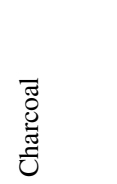 & 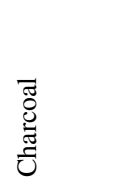 & 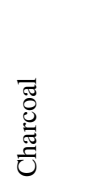 \\
\hline 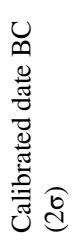 & $\begin{array}{l}\frac{9}{d} \\
\infty \\
0 \\
1 \\
\\
a\end{array}$ & 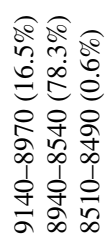 & 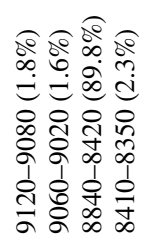 & $\begin{array}{l}2 \\
2 \\
\infty \\
1 \\
\infty \\
\infty \\
\infty\end{array}$ & 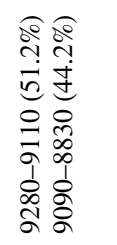 & 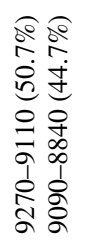 & $\begin{array}{l}0 \\
\infty \\
\infty \\
0 \\
0 \\
\swarrow 1 \\
\alpha\end{array}$ & $\begin{array}{l}0 \\
\infty \\
\infty \\
1 \\
1 \\
\curvearrowleft \\
\sigma\end{array}$ & 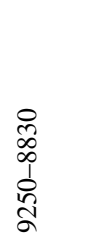 \\
\hline 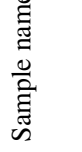 & $\begin{array}{l}\overrightarrow{0} \\
\underset{1}{1}\end{array}$ & 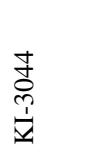 & 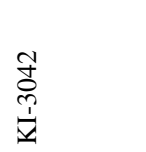 & 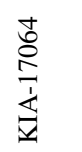 & 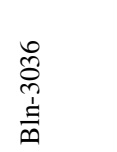 & 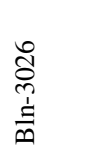 & 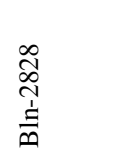 & 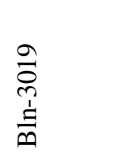 & $\begin{array}{c}\text { ठิ } \\
\text { के } \\
\stackrel{1}{=}\end{array}$ \\
\hline 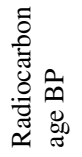 & $\begin{array}{l}8 \\
\text { +1 } \\
0 \\
2 \\
a\end{array}$ & $\begin{array}{l}0 \\
\infty \\
+1 \\
0 \\
+ \\
0\end{array}$ & $\begin{array}{l}\infty \\
+1 \\
+\infty \\
\infty \\
\approx\end{array}$ & $\begin{array}{l}m \\
\cdots \\
+1 \\
2 \\
\approx\end{array}$ & $\begin{array}{l}\stackrel{2}{1} \\
+1 \\
0 \\
\& \\
0\end{array}$ & $\begin{array}{l}8 \\
+1 \\
0 \\
8 \\
8\end{array}$ & $\begin{array}{l}\stackrel{2}{+1} \\
+1 \\
0 \\
0\end{array}$ & 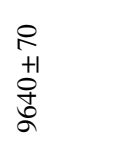 & \begin{tabular}{l}
8 \\
0 \\
+1 \\
0 \\
\multirow{2}{0}{} \\
0
\end{tabular} \\
\hline 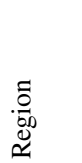 & 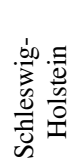 & 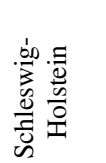 & 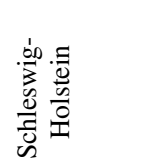 & 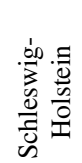 & 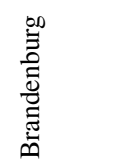 & 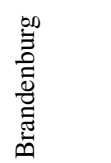 & 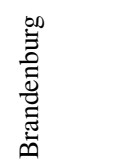 & 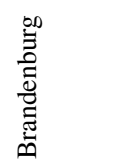 & 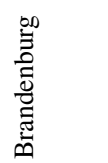 \\
\hline$\stackrel{\mathscr{G}}{\mathscr{\omega}}$ & 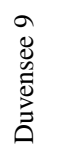 & 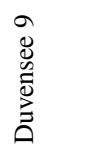 & 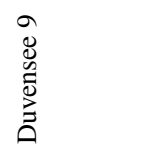 & 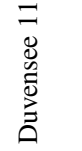 & 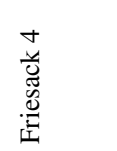 & 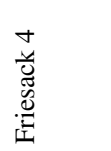 & 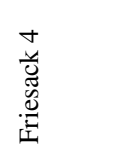 & 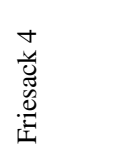 & 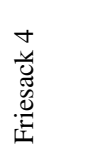 \\
\hline$\dot{z}$ & $\ddot{\lambda}$ & $\underset{\sim}{J}$ & $\approx$ & $\stackrel{\sim}{\sim}$ & $\hat{\lambda}$ & $\stackrel{\infty}{\sim}$ & ฉે & in & $\vec{m}$ \\
\hline
\end{tabular}




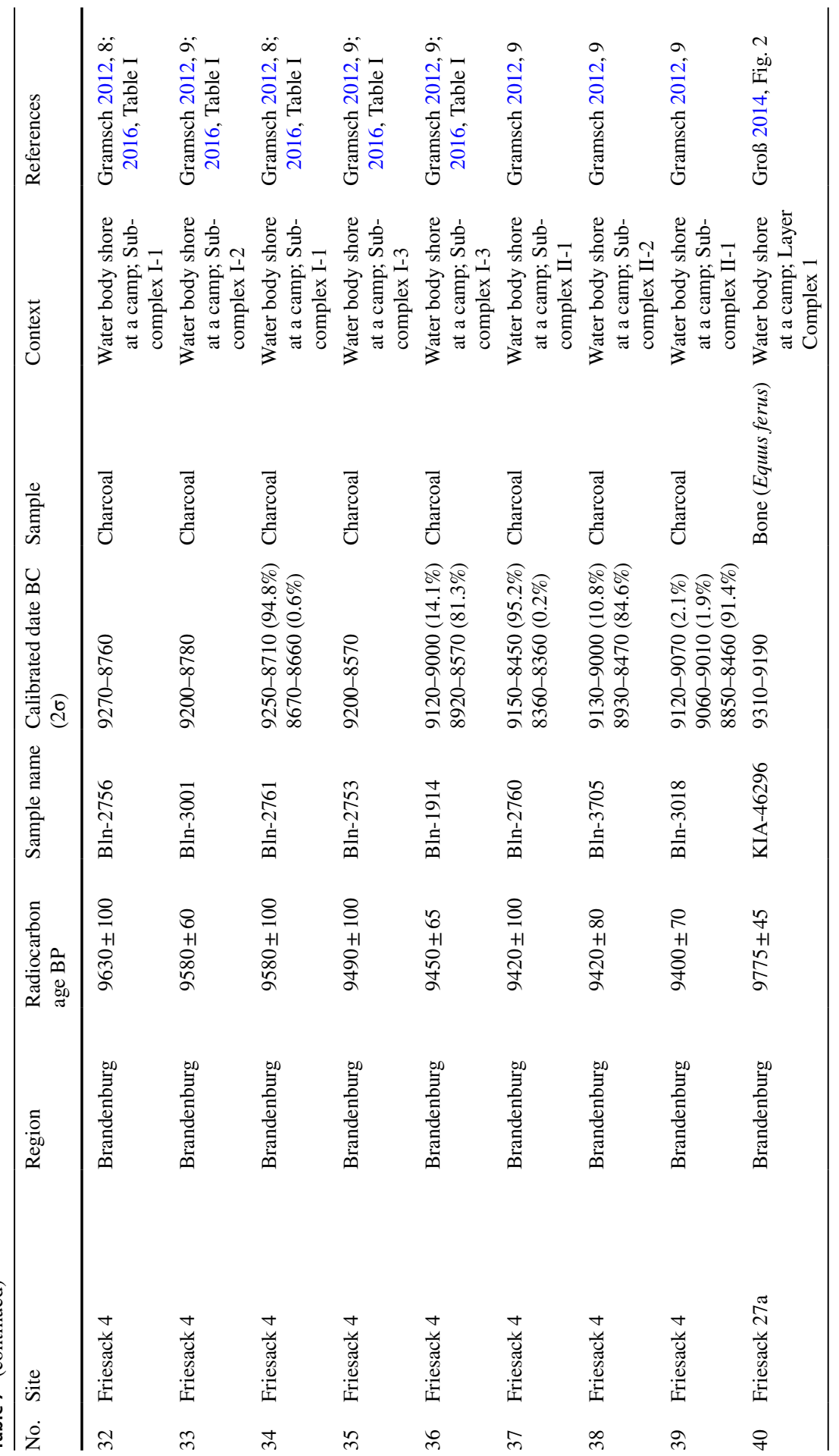




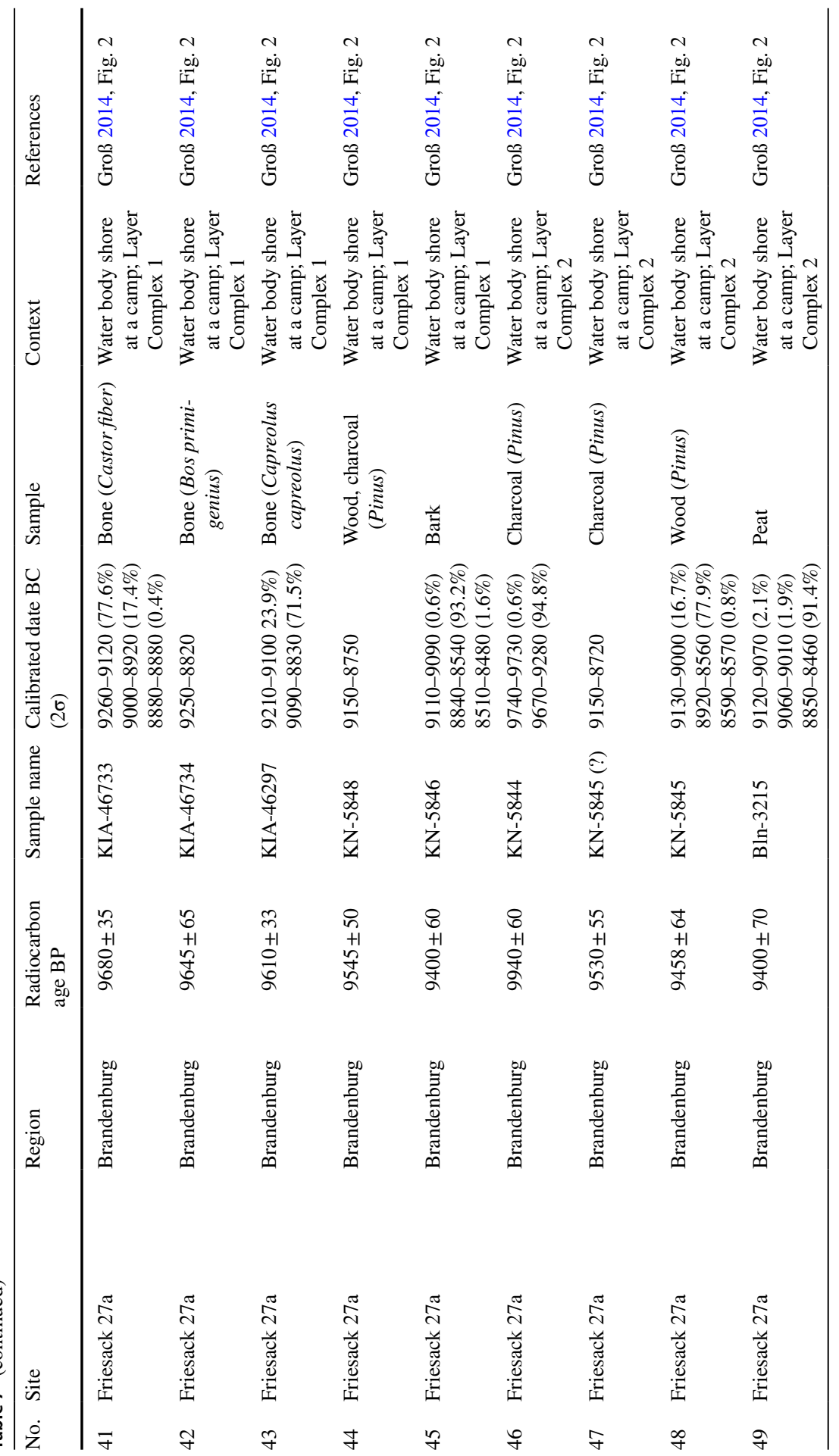




\begin{tabular}{|c|c|c|c|c|c|c|c|c|c|c|}
\hline 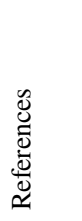 & 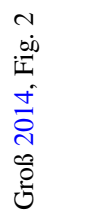 & 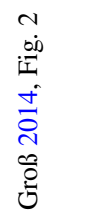 & 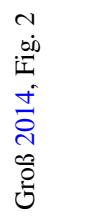 & 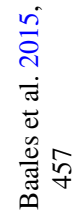 & 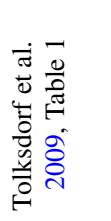 & 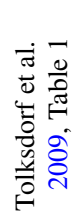 & 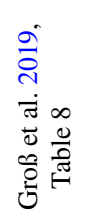 & 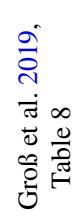 & 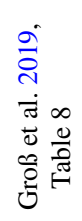 & 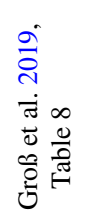 \\
\hline 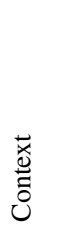 & 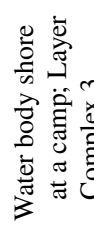 & 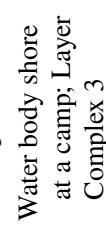 & 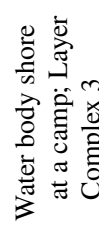 & $\begin{array}{l}\vec{B} \\
\text { 胥 } \\
\text { 品 }\end{array}$ & 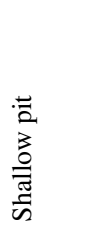 & 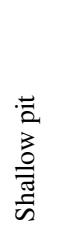 & 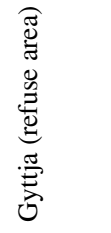 & 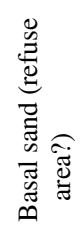 & 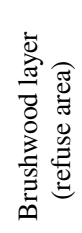 & 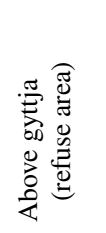 \\
\hline 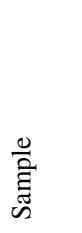 & 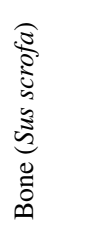 & 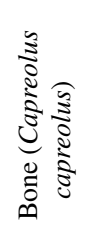 & 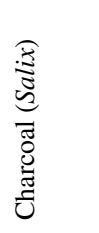 & 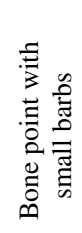 & 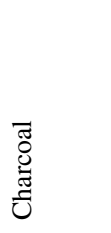 & 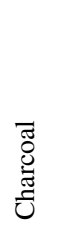 & 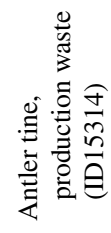 & 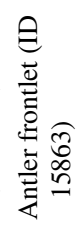 & 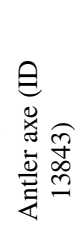 & 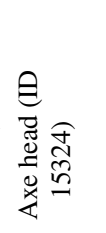 \\
\hline 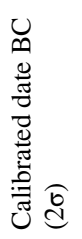 & 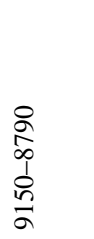 & 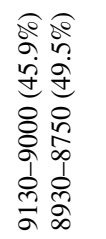 & 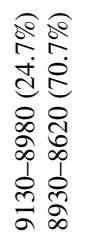 & $\begin{array}{l}0 \\
0 \\
\infty \\
1 \\
1 \\
\infty \\
\infty \\
\infty\end{array}$ & 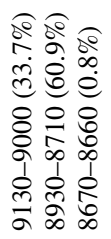 & 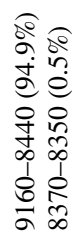 & 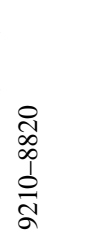 & 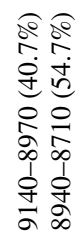 & 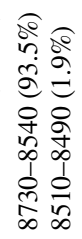 & 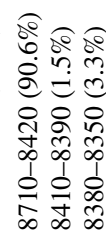 \\
\hline 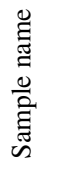 & 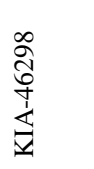 & 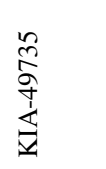 & \begin{tabular}{l}
$m$ \\
\multirow{2}{*}{} \\
$\sim$ \\
$\dot{z}$ \\
$z$
\end{tabular} & $\sum_{\substack{n \\
\infty}}^{\infty}$ & 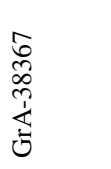 & $\begin{array}{l}\stackrel{8}{0} \\
\text { } \\
\stackrel{1}{1} \\
\dot{1}\end{array}$ & 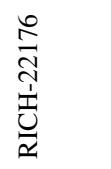 & 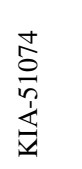 & 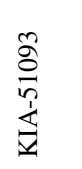 & 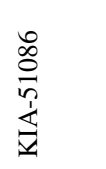 \\
\hline 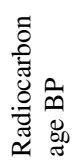 & $\begin{array}{l}8 \\
+ \\
+1 \\
b \\
o \\
a\end{array}$ & $\begin{array}{l}m \\
+1 \\
\frac{n}{n}\end{array}$ & $\begin{array}{l}8 \\
0 \\
+1 \\
0 \\
\infty \\
0\end{array}$ & 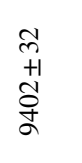 & $\begin{array}{l}q \\
+ \\
+1 \\
b \\
o \\
a\end{array}$ & 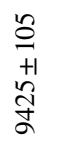 & $\begin{array}{l}+ \\
+ \\
+1 \\
\infty \\
0 \\
0\end{array}$ & $\begin{array}{l}0 \\
+ \\
+1 \\
\infty \\
\ddot{n}\end{array}$ & $\begin{array}{l}n \\
m \\
+1 \\
a \\
\sigma \\
\sigma\end{array}$ & $\begin{array}{l}\vec{n} \\
+1 \\
o \\
o \\
\sigma\end{array}$ \\
\hline 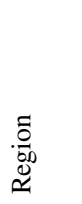 & 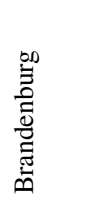 & 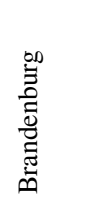 & 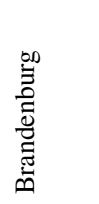 & 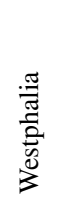 & 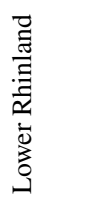 & 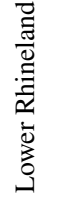 & 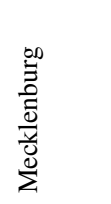 & 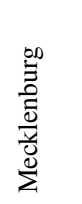 & 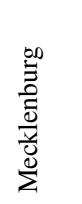 & 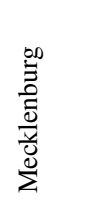 \\
\hline$\stackrel{\cong}{\check{n}}$ & 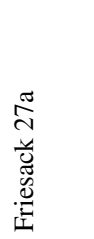 & 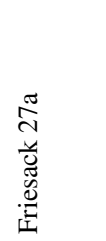 & 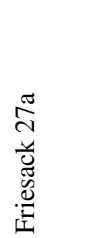 & 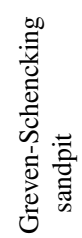 & 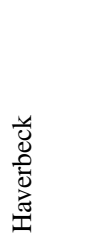 & 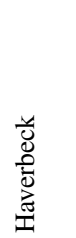 & $\begin{array}{l}\vec{\Xi} \\
\overline{0} \\
\overline{0} \\
0 \\
0 \\
\overline{0} \\
0 \\
0\end{array}$ & 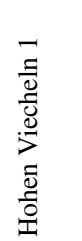 & $\begin{array}{l}\bar{\Xi} \\
\bar{\Xi} \\
\overline{0} \\
0 \\
0 \\
\overline{0} \\
\overline{0} \\
0\end{array}$ & 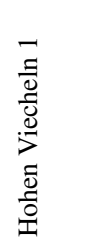 \\
\hline 8 & in & $\vec{n}$ & in & $n$ & in & $\approx n$ & in & in & $\stackrel{\infty}{\sim}$ & nิ \\
\hline
\end{tabular}




\begin{tabular}{|c|c|c|c|c|c|c|c|c|c|c|c|}
\hline 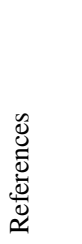 & 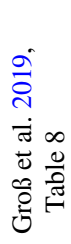 & 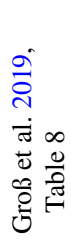 & 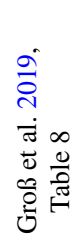 & 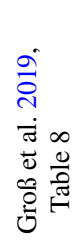 & 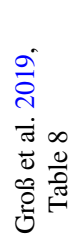 & 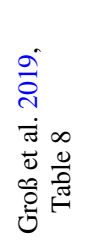 & 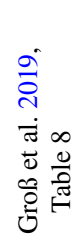 & 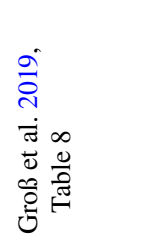 & 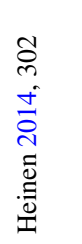 & 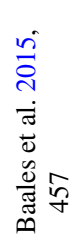 & 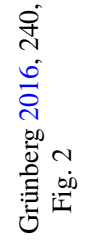 \\
\hline $\begin{array}{l}\overrightarrow{\text { U. }} \\
\stackrel{0}{0}\end{array}$ & 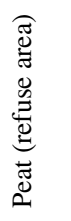 & 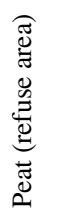 & 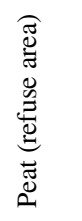 & 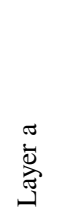 & 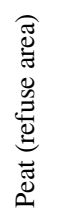 & 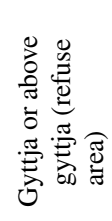 & 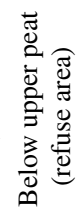 & 芯 & 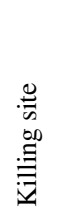 & $\begin{array}{l}\vec{\Xi} \\
\vec{E} \\
\vec{\Xi}\end{array}$ & 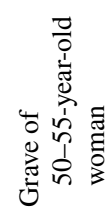 \\
\hline 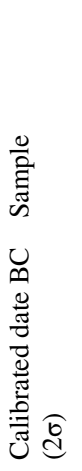 & 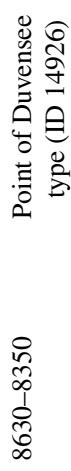 & 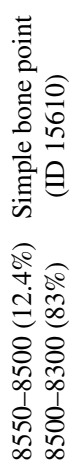 & 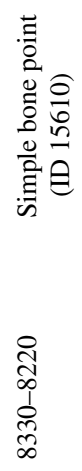 & 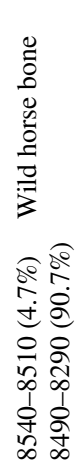 & 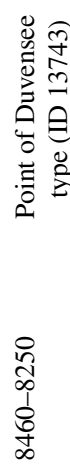 & 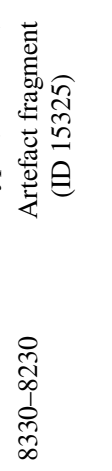 & 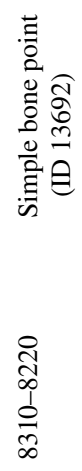 & 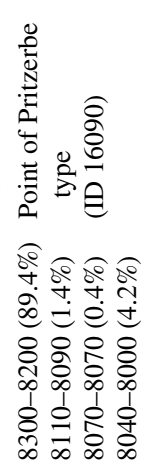 & 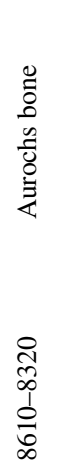 & 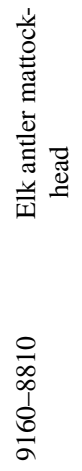 & 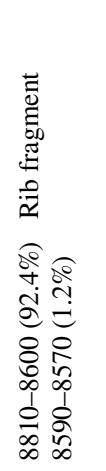 \\
\hline 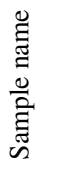 & 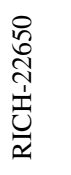 & 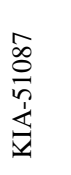 & 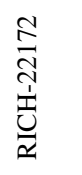 & 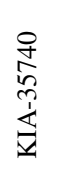 & 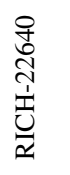 & 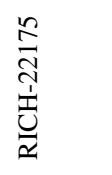 & 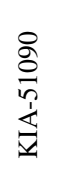 & 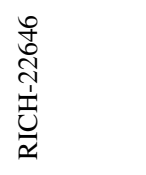 & 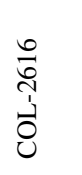 & $\sum_{\Sigma}^{\dot{n}} \frac{\partial}{\exists}$ & 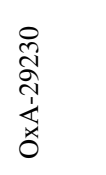 \\
\hline 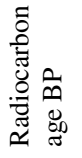 & 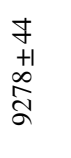 & $\begin{array}{l}\underset{f}{+} \\
+1 \\
\stackrel{+}{o} \\
\sigma\end{array}$ & $\begin{array}{l}\forall \\
+1 \\
+1 \\
2 \\
2\end{array}$ & $\begin{array}{l}q \\
+ \\
+1 \\
\infty \\
a \\
a\end{array}$ & $\begin{array}{l}a \\
+ \\
+1 \\
o \\
a\end{array}$ & $\begin{array}{l}\stackrel{f}{+} \\
+1 \\
\stackrel{+}{\$} \\
\&\end{array}$ & $\begin{array}{l}\mathcal{Y} \\
+1 \\
+1 \\
\stackrel{2}{8}\end{array}$ & $\begin{array}{l}\stackrel{9}{+} \\
+1 \\
\stackrel{2}{8} \\
\&\end{array}$ & 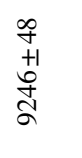 & $\begin{array}{l}o \\
\text { n} \\
+1 \\
a \\
a \\
a\end{array}$ & $\begin{array}{l}\text { I } \\
+1 \\
\stackrel{+}{y} \\
\text { g }\end{array}$ \\
\hline 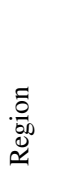 & 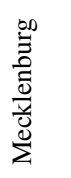 & 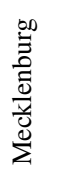 & 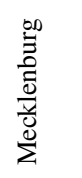 & 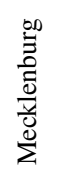 & 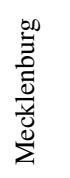 & 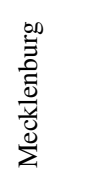 & 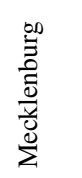 & 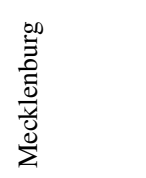 & 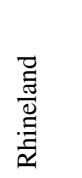 & $\begin{array}{l}\frac{\pi}{7} \\
\frac{\pi}{2} \\
\frac{0}{0} \\
3\end{array}$ & 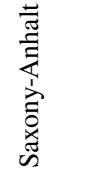 \\
\hline$\stackrel{\mathscr{n}}{\omega}$ & 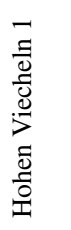 & 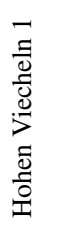 & 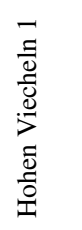 & $\begin{array}{l}\vec{\Xi} \\
\overline{0} \\
\overline{0} \\
0 \\
\overline{0} \\
\overline{0} \\
\overline{0} \\
01\end{array}$ & 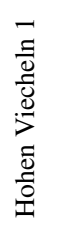 & 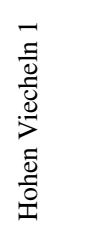 & 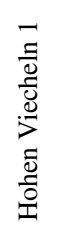 & 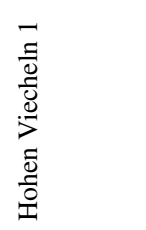 & 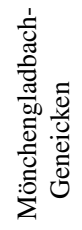 & 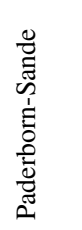 & $\begin{array}{l}\text { on } \\
\vdots \\
0 \\
0 \\
0 \\
5 \\
5\end{array}$ \\
\hline$z^{\circ}$ & 8 & $\vec{b}$ & ชิ & $\tilde{6}$ & $\vec{b}$ & $\sqrt{6}$ & 8 & $\widehat{6}$ & $\ddot{b}$ & gิ & 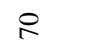 \\
\hline
\end{tabular}




\begin{tabular}{|c|c|c|c|c|}
\hline 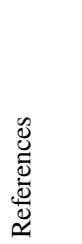 & 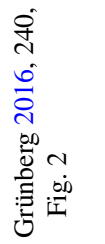 & 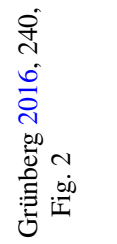 & 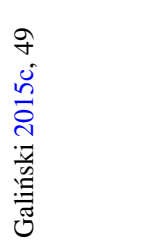 & 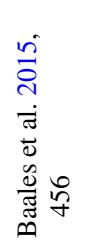 \\
\hline $\begin{array}{l}\overrightarrow{\widetilde{x}} \\
\stackrel{\vec{U}}{0}\end{array}$ & 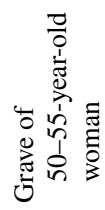 & 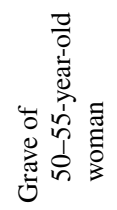 & o. & 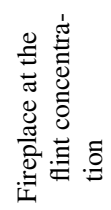 \\
\hline 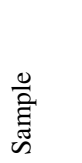 & 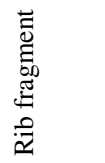 & 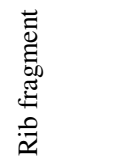 & & 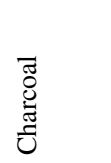 \\
\hline 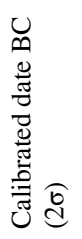 & $\begin{array}{l}? \\
n \\
\infty \\
0 \\
0 \\
\infty \\
\infty \\
\infty\end{array}$ & $\begin{array}{l}8 \\
0 \\
\infty \\
0 \\
0 \\
0 \\
\infty\end{array}$ & 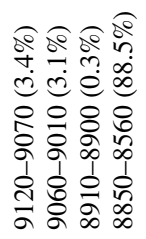 & 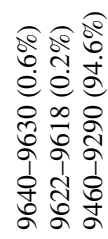 \\
\hline 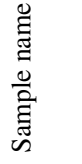 & 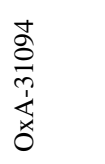 & $\frac{n}{o}$ & 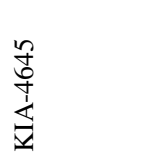 & 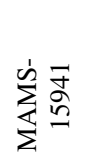 \\
\hline 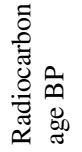 & \begin{tabular}{l}
$n$ \\
$\stackrel{7}{+1}$ \\
+1 \\
0 \\
\multirow{a}{*}{}
\end{tabular} & $\begin{array}{l}q \\
+1 \\
\stackrel{+}{\alpha} \\
\sigma\end{array}$ & \begin{tabular}{l}
8 \\
8 \\
+1 \\
0 \\
\multirow{9}{0}{}
\end{tabular} & $\begin{array}{l}\tilde{2} \\
+1 \\
\tilde{n} \\
\Omega\end{array}$ \\
\hline 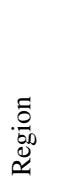 & 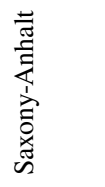 & 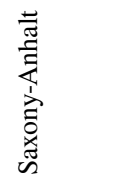 & 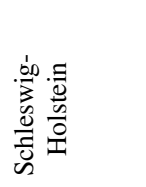 & 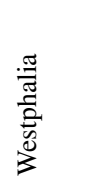 \\
\hline 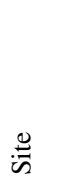 & 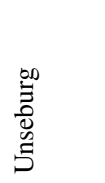 & $\begin{array}{l}\text { on } \\
\vdots \\
0 \\
0 \\
\vdots \\
5\end{array}$ & 咅 & 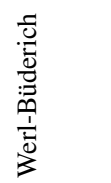 \\
\hline 8 & $\nabla$ & $\mathbb{N}$ & $\stackrel{r}{r}$ & t \\
\hline
\end{tabular}


Assuming continuity inside each phase, there are three possible combinations of their mutual relationship (Bronk Ramsey 2009a):

- sequential - the phases succeed one another, with possibility of hiatus between them;

- contiguous - the phases directly succeed one another, with no interruption;

- overlapping - the order of the phases is unknown, or one phase begins before the other ends.

To attempt to answer the above questions, we proceeded as follows. First, three models were designed-each with two phases (groups of dates) associated with the Final Palaeolithic and the Mesolithic. Codes of all processed models and their results are included in Electronic Supplementary Material 1. Each model represented one of the three possible combinations outlined above. These were the null hypothesis; the results of model processing led to either their acceptance or rejection. Each model was run twice, first as an outlier analysis to reject dates not fitting the statistical model (Bronk Ramsey 2009b). The same parameters were used in analysis of all three hypotheses. The parameter used for the whole model was Outlier_Model(“General”,T(5),U(0,4),"t'), for every analysed date the assumption was that the probability of it being an outlier was 0.05 . The second run was identical, but with the dataset purged of previously identified outliers. The results of all models were evaluated for 2 sigma (95.4\%), with all values rounded to the nearest 10 .

\section{The Boundary Between the Final Palaeolithic and Mesolithic in Light of Radiocarbon Dating}

\section{The Polish Plain}

In the sequential model the null hypothesis was that of two discrete phases in human settlement, with a hiatus between Final Palaeolithic and Mesolithic communities. The modelling included the start and end boundaries for the phases, as well as the length of the gap between them.

The first stage — outlier analysis—revealed the need to eliminate date MKL-3349 from Bolków 1. After its removal from the dataset the model was run again. The query interval (i.e. the length of the hiatus) produced a range of 0-310 years. This result, with 0 as the value of one of the boundaries, makes it imperative that the hypothesis of a hiatus between the phases should be rejected.

The above analysis eliminated the hypothesis of a hiatus between the two settlement phases but did not resolve the issue of possible overlap. Thus the next, overlapping model was designed with the assumption that the phases might overlap. Hence the model now included the query order, which would calculate the relative sequence of modelled events.

Here again outlier analysis pointed out the need to eliminate date MKL-3349, and after its removal the model was run again. The model demonstrated high consistency 
Table 8 Methods of radiocarbon analysis of samples from the early Mesolithic sites in the North German Plain

\begin{tabular}{|l|c|c|c|}
\hline \multicolumn{1}{|c|}{ Dates } & $\begin{array}{l}\text { Radiocarbon dates } \\
\text { without AMS }\end{array}$ & $\begin{array}{l}\text { AMS radiocarbon } \\
\text { dates }\end{array}$ & Number \\
\hline Graves & - & 3 & 3 \\
\hline Stratified sites & 43 & 18 & 61 \\
\hline $\begin{array}{l}\text { Flint } \\
\text { concentrations } \\
\text { in sandy areas }\end{array}$ & 1 & 3 & 4 \\
\hline $\begin{array}{l}\text { Stray } \\
\text { antler/bone } \\
\text { artefacts }\end{array}$ & - & 5 & 5 \\
\hline Killing site & - & & 1 \\
\hline Number & 44 & 30 & 74 \\
\hline
\end{tabular}

(c. $118 \%$ for indexes $\mathrm{A}_{\text {model }}$ and $\mathrm{A}_{\text {overall }}$ ), in spite of one of the dates (Bolków, site 1-MKL-3351) generating Index A below the required level (53\%). The result of the Order query, visualised using prob_phases_relat.r script (Alberti 2016) points to the probability of the phases' overlap being relatively low $(11 \%$, i.e. with $89 \%$ probability of their being separated)-hence the overlapping hypothesis should be rejected (Figs. 2, 3). This model also identified the points at which Final Palaeolithic settlement ended (between 9290 BC and 9030 BC) and the Mesolithic began (between $9230 \mathrm{BC}$ and $8860 \mathrm{BC}$ ).

The two procedures described above allowed the elimination of the hypothesis of there being either a hiatus or an overlap between Final Palaeolithic and Mesolithic settlement. The third stage of modelling, that is the contiguous model, aimed at confirmation or rejection of the hypothesis of a 'smooth' passage between the two: the modelling aimed at identification of the period when one phase transitioned into the other.

Yet again outlier analysis led to the elimination of date MKL-3349 from the dataset. When run without it, the model revealed a high level of consistency, thus supporting its high statistical accuracy. The modelling generated the value of the boundary function - that is, the time range during which the Final Palaeolithic 'transitioned' into the Mesolithic-placing it between 9260 BC and 8980 BC (Fig. 2). Some difference in the chronology of the Early Mesolithic may be discerned between the western and eastern areas of the Polish Plain (Fig. 4). Separate modelling of boundary dates for the onset of the early Mesolithic for these two areas (using the overlapping model) produced the result that in the west of the Polish Plain the Mesolithic began between $9410 \mathrm{BC}$ and $8860 \mathrm{BC}$, and in the eastern part-between $9230 \mathrm{BC}$ and $8760 \mathrm{BC}$. Hence it seems reasonable to assume that the transition to the Mesolithic way of life in the west of present-day Poland happened at least several decades earlier.

The analysis described above allows us to reject the hypothesis of there being either a hiatus or overlap between Final Palaeolithic and Mesolithic settlement on the plains of Poland. The existing dating points to the veracity of the hypothesis that there was a smooth transition between the two phases, with this event taking 


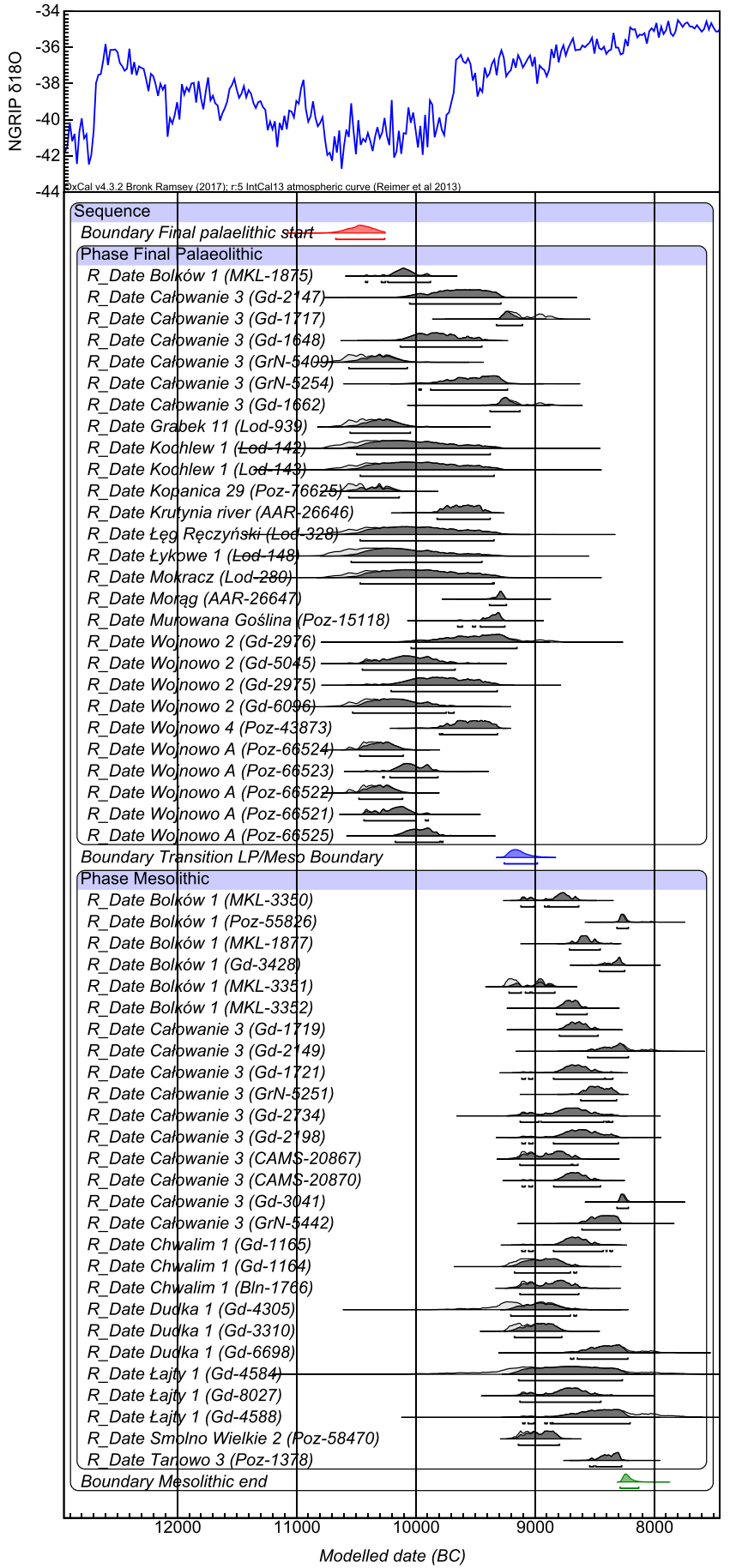

Fig. 2 The model of the Palaeolithic/Mesolithic boundary in the light of modelling radiocarbon dates from the Polish Plain 
place some time between $9260 B C$ and 8980 BC (Supplementary Material 2). For this reason we would describe such a model as the replacement model.

\section{The North German Plain}

The result of the same analysis for data from Germany was different. Here again three possible variants were modelled, and each model run twice-the first run to eliminate outliers from the dataset. Further outlier analysis led to the exclusion of dates from Bützsee-Altfriesack (OxA-8742, OxA-8743) and Bedburg-Königshoven (KN-4139, KN-4138, KN-4137, KN-4136) from further calculations (Fig. 5). The subsequent modelling of the three relationship variants determined that the most probable outcome for this area was the two phases overlapping.

The modelling results run for Germany point to the Final Palaeolithic and Mesolithic there overlapping as the end of the Final Palaeolithic takes place between 9210 $B C$ and $8900 B C$, while the Mesolithic begins somewhere between $9400 B C$ and 9300 BC (Supplementary Material 2). Compared with the Polish Plains, in Germany the end of Final Palaeolithic settlement happened at the same time as in Poland, but Mesolithic related settlement appeared some 200 years earlier. For this reason we would describe such a model as the coexistence model.
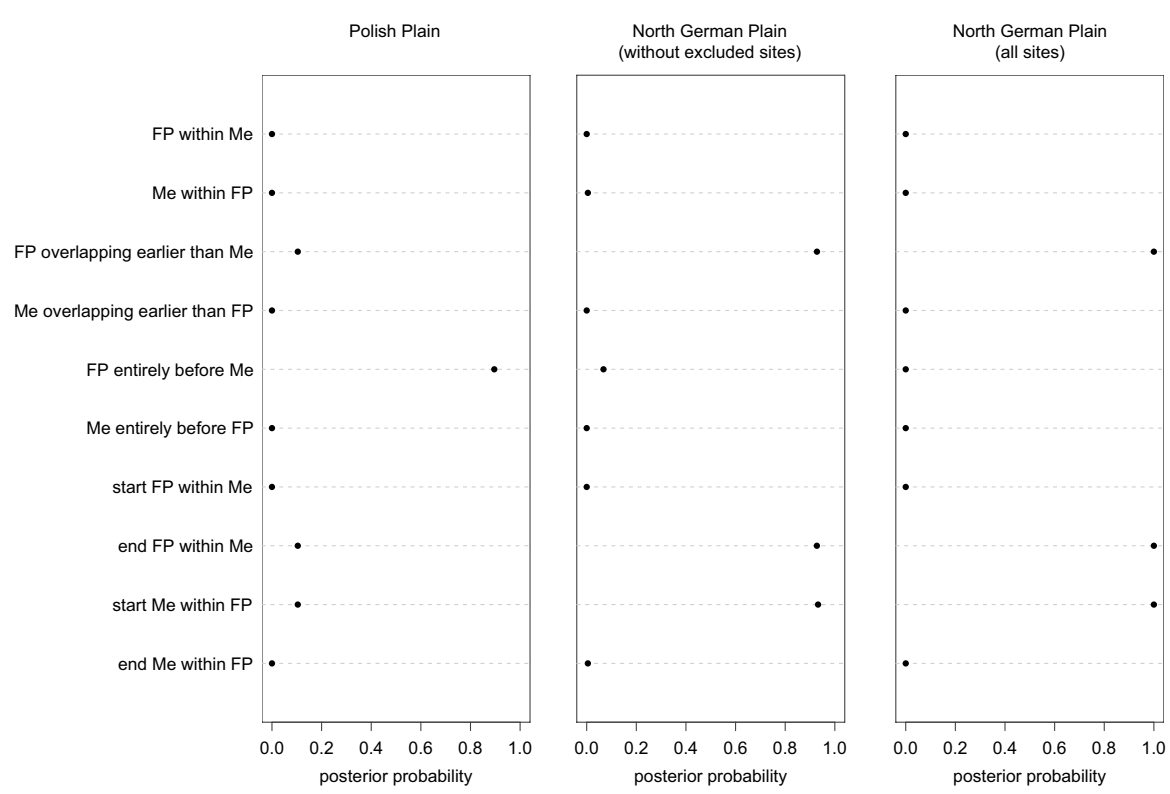

Fig. 3 The posterior probabilities of modelling Palaeolithic/Mesolithic boundary in the Polish Plain and in the North German Plain and the Eifel 


\section{Discussion}

\section{The Pleistocene-Holocene Transition and Cultural Change}

Prevailing scientific opinion on the end of the Final Palaeolithic and the emergence of the early Mesolithic in the Polish Plain is that this process was significantly shaped by human migrations. This assumption has its roots in the first half of the $20^{\text {th }}$ century, and survived the emergence of radiocarbon dating. It was in large part influenced by early stylistic studies of flint artefacts, focusing on their typology and not on the technology of their manufacture. The first works on the genesis of the Mesolithic_-which are today of purely historical interest-pointed out the similarities between Mesolithic artefacts from Poland and those of the western European Tardenoisian (Kostrzewski 1939; Zotz 1934); accordingly their appearance in Poland was linked with an influx of human populations from the west of the continent. This view was rejected by Polish scholars in the mid-twentieth century (Schild and Więckowska 1961).

The discussion on the end of Final Palaeolithic and the dawn of the early Mesolithic on the plains of Poland is still dominated by the migration theory, a hypothesis which in general terms holds that Swiderian people moved to northeastern Europe while a portion of Ahrensburgian hunters moved to Scandinavia (Galiński 1997; 2002; Ginter 1973; Kabaciński 2016; Kabaciński and Sobkowiak-Tabaka 2010; Kobusiewicz 1999; J. K. Kozłowski 2001; Kozłowski and Kaczanowski 2003; Kozłowski and Kozłowski 1975, 1977; S. K. Kozłowski 1969; 1972; Schild 1996a, 1996b, 2001; Sobkowiak-Tabaka 2011, 2016; Więckowska 1975). Some researchers interpret radiocarbon dates from Polish sites as indicating a hiatus between Final Palaeolithic and early Mesolithic settlement (Kabaciński 2016; Schild 1996a, 1996b, 2001) where, after a period of some 150-300 years, during which the Polish Plain was a depopulated desert, it was resettled by an inflow of Mesolithic people. Romuald Schild sees evidence for migrations and a settlement hiatus in the collapse

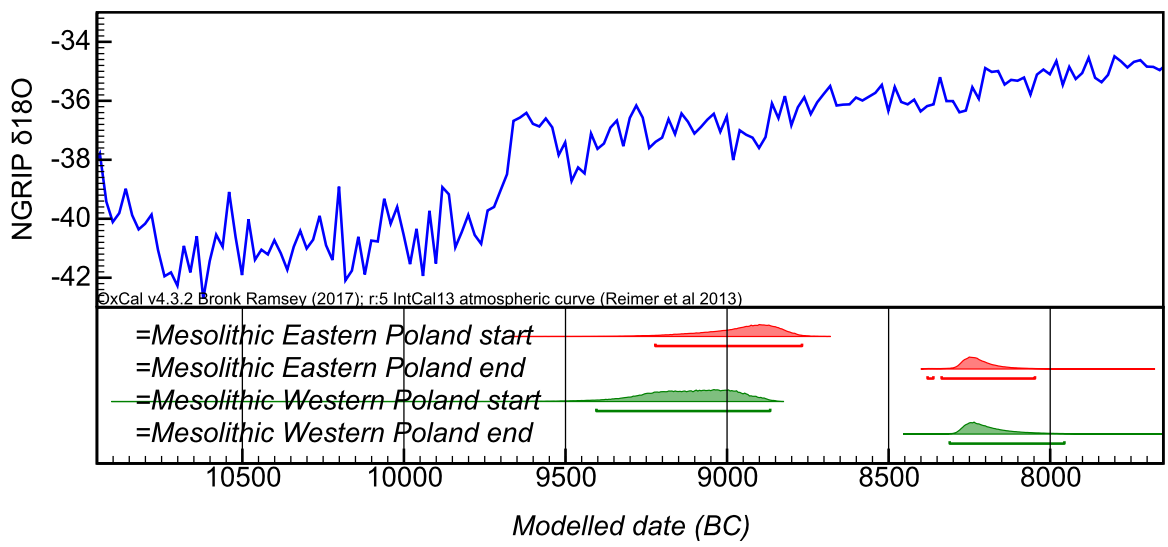

Fig. 4 The model of the Palaeolithic/Mesolithic boundary in the eastern and western area of the Polish Plain 
of chocolate flint exploitation in the early Mesolithic; in the scant signs of early Mesolithic settlement around the red pigment mine at Rydno; and in the severance of Final Palaeolithic exchange networks, whose existence is indicated by wide distribution of both commodities. In his opinion, this would point to the Mesolithic population being unaware of these resources, eo ipso being distinct from Swiderian communities. Most researchers consider dates from early Mesolithic sites from eastern Germany to pre-date those from Poland, thus hinting at Mesolithic ideas coming to Poland from that direction; nevertheless, there is a group championing the idea that inflow from the South trumped that from the West. T. Galiński (1992, p. 160 ) is more reserved in his views, as he considers the cultural transformation at the transition from Pleistocene to Holocene to have been a complex process: changes inside Final Palaeolithic cultures took place in parallel to the immigration of groups bearing 'Mesolithic culture'. In the opinion of S. K. Kozłowski and T. Galiński, the Mesolithic in Polish lands, besides a component characteristic of the Ahrensburgian (cf. Kozłowski 1972, p. 222), includes artefacts suggesting the possibility of immigration from the Carpathian Basin of people representing Epi-Gravettian, Epi-Tardigravettian and/or Epi-Magdalenian traditions (Kozłowski 1989, p. 136; Galiński 2002, p. 350). For J. K. Kozłowski (2001, p. 117) the development of the Mesolithic in today's Poland was too rapid to be explicable with reference to adaptation, hence Mesolithic communities must have come to the Polish Plain from the West during the Preboreal. However, in his opinion it was possible that in western Poland the traditions of the Arch-backed Point Complex lived on, with these communities giving rise to the early Mesolithic culture (Kozłowski and Kaczanowski 2003, p. 101). M. Kobusiewicz (1999, p. 117) also allows for the possibility of the emergence of the Mesolithic in northwestern Poland to be partly autochthonous in origin, with a contribution of immigrants from the West.

Many authors point out that in various parts of Poland there are Final Palaeolithic assemblages from the early Preboreal. According to S. K. Kozłowski (1969), in northeastern Poland one may discern a Preboreal phase of the Swiderian which did not, however, extend to the west of the Vistula. In such a case one may presume that Swiderian reindeer hunters would also be present in today's Latvia and Estonia (Zagorska 2006).

The few existing radiocarbon dates for northern Germany and the Rhineland do not provide a clear picture as to the boundary between the Palaeolithic and Mesolithic. According to many German scientists, the cultural Final Palaeolithic/Mesolithic division closely follows the climatostratigraphical boundary between the Pleistocene and Holocene, that is, the onset of the Holocene and of the Mesolithic are as good as synonymous, with radiocarbon dating showing that Final Palaeolithic cultures survived into the Preboreal being erroneous (Street et al. 2001, p. 393). The theory of migration of Final Palaeolithic hunters is considered to apply to the North German Plain as well (Groß 2014; Terberger 2004, 2006), with an impreciselydefined segment of Ahrensburgian hunters leaving this area and heading North in pursuit of reindeer herds, although such communities are presumed to still be present in situ in the early phases of the Preboreal (Groß 2014, p. 220; Terberger 2004, p. 44). However, the analysis of radiocarbon dates supports the thesis that for the vast expanses of the European Plain and neighbouring uplands, no uniform process - or 
Fig. 5 The model of the Palaeolithic/Mesolithic boundary in the light of modelling radiocarbon dates from the North German Plain and the Eifel

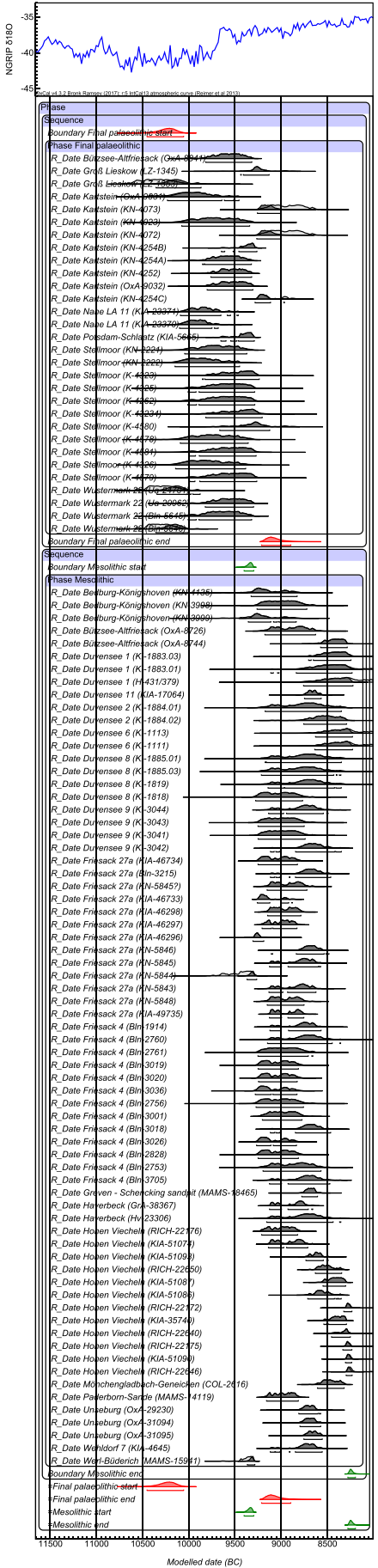


model - of changes existed, that it was more a patchwork of many local models of the disappearance of old and emergence of new cultural trends. The dynamics of such changes are affected not only by local environmental conditions, but also by complex cultural and social relationships. The most recent literature on the subject appears to be dominated by the view that the transition from the Final Palaeolithic to the Mesolithic in the North European Plain was a complex process, involving the existence of human groups following different cultural traditions (Groß 2014; Sørensen et al. 2018; Zander 2016). Here D. Groß (2014, p. 219) draws upon earlier migration theories, according to which Ahrensburgian reindeer hunters followed that species north, while the forerunners of the Mesolithic_-most probably from the Federmesser milieu-slowly drifted northward, following the Preboreal succession of woodlands.

The transition from the Final Palaeolithic to the Mesolithic in southern Scandinavia also is the subject of lively debate. According to E. Brinch Petersen (2009), currently available data points to the Maglemosian beginning with the onset of the Holocene, there being no reason to assume the existence of the Ahrensburgian during the Preboreal. However, not all researchers agree with this opinion as, in spite of the lack of radiocarbon dates from early Preboreal Final Palaeolithic sites, one might assume that the transition was not purely linear, that is, that the Ahrensburgian was immediately followed by the Maglemosian (Sørensen et al. 2018, p. 328). There are many pointers to the early Maglemosian in present-day Denmark and southern Sweden being accompanied by other communities, with, for example, Long Blade Industries or the Epi-Ahrensburgian (Nørregård VI, Årup context 1). It is possible that this variety was related to different biotopes and landscape types (e.g. Jutland $v s$. Sealand), and the various human groups 'were mobile in relation to seasons and game' (Sørensen et al. 2018, p. 328).

We find an equally complex situation in Westphalia where, as in other parts of Europe, during the transition from the Pleistocene to the Holocene one may note the coexistence of various cultural traditions, including Long Blade Industries known from the northwest of the continent (Baales et al. 2015; Zander 2016). In the HeekNienborg archaeological site long blades are accompanied by finds of tools typical for the Final Palaeolithic and Mesolithic: Zonhoven points, a double truncation, a Komornica point and an isosceles triangle (Zander 2016). Sadly, no radiocarbon dating was possible for this site. Inventories from northwestern France containing long blades and microliths were found at Buhat and Alizay, with the latter settlement being dated to c. 9700-9000 cal BC.

The analysis of radiocarbon dating results does not allow for the rejection of the thesis that Final Palaeolithic and Mesolithic settlement in the Polish Plains were not separated - as presumed to date-by a hiatus; however, the modelling of radiocarbon dates points to the two phases of human habitation directly following one another. The probability that for some time the two cultures existed side by side, that is, overlapped, is very low. This model fits the way the Mesolithic developed in the Polish Plains. One cannot rule out population movements, but these could not have been mass migrations involving larger numbers of people. The Final Palaeolithic cultural model was still around at the onset of the Preboreal. 
We now turn to the overlap between the Final Palaeolithic and the dawn of the Mesolithic in the North German Plain. As already mentioned, the overlap model is generated by the large number of Final Palaeolithic dates from Stellmoor and Kartstein. If these were to be eliminated-as suggested by some German researchers-this would drastically reduce the number of radiocarbon dates from Final Palaeolithic sites, and in such a scenario the probability of overlap becomes very low. According to German scientists who hold that view (Street et al. 2001), these dates should be rejected as their connection to the Final Palaeolithic is doubtful. Similar doubts may be extended towards a series of early dates from Mesolithic sites at Bedburg-Königshoven and Bützsee-Altfriesack. Two additional models were developed so as to examine the impact of the questioned dates (Fig. 6): the first excluded dates from the aforementioned Mesolithic sites (Fig. 6b), the second additionally excluded dates from the listed Palaeolithic sites (Fig. 6c). In both cases the result of the modelling is affected by the diminished number of dates for both phases, in effect producing greatly extended boundaries for the end of the Final Palaeolithicand indirectly lowering the accuracy of statistical conclusions based upon those models. Irrespective of that fact, both models confirm the previous conclusion that the two phases overlapped. The sole difference concerns the probable timing of the demise of Final Palaeolithic settlement: when all dates are included in the dataset (Fig. 6a, b) this could have happened between 9210 BC and $8900 \mathrm{BC}$, whereas when the dates from Stellmoor and Kartstein are excluded, the boundary is moved up by some 200 years, to a period lasting from $9430 \mathrm{BC}$ to $8810 \mathrm{BC}$. In either case there should be a period of at least two hundred years during which both cultural traditions existed side-by-side in the North German Plain.

It is worth pointing out that the modelling makes Mesolithic sites from Poland younger than the oldest German sites, a result consistent with R. Schild's calculations (1996b, 2001). However, on closer inspection of the differences in dating we note that the onset of the Mesolithic in the west of Poland is dated similarly to its emergence in the German Plains, with the difference in chronology being between the appearance of the Mesolithic in the western and eastern halves of Poland. However, other data make it difficult to assume that this time lag reflects the pace of West to East migration of Mesolithic communities. Date modelling has shown that there was no hiatus between Final Palaeolithic and Mesolithic settlement. At present it is difficult to interpret this difference in timing-it could be related to the existence of a more continental type of climate in the eastern reaches of Poland. A role could have been played by the cooling of the climate in the early Preboreal period, which in eastern Poland began c. $9150 \mathrm{cal}$ BC and lasted for some 60-160 years (de Klerk et al. 2007); the cooling might possibly have been more acute than in western Poland, thus delaying the spread of the Mesolithic way of life.

In our opinion the issues of possible hiatus and migration should be addressed separately and with consideration of environmental, cultural and societal factors. Below we examine the onset of the Mesolithic in Poland from different points of view: in the first section we look early Holocene environmental changes and their pace; in the second section we ponder the message communicated by lithics and artefacts of symbolic culture; and in the third section we cover the social aspects of the beginnings of the Mesolithic, with special focus on hunter-gatherer migrations. 


\section{Environment}

The transition from the Pleistocene to the Holocene in the Polish Plain involves profound changes in the natural environment. From the perspective of local communities, the most significant change was the warming of the climate and the resultant appearance of woodlands and associated fauna, accompanying the intensive melting of dead ice, which created inland expanses of water. Although reliable environmental data for climate change accompanying the early Holocene in Central and northern Europe exist, the pace and extent of the changes are subject to debate. For example at Lundby in Sealand dense woodland appears only at c. $9300 \mathrm{cal}$ BC, replacing open woodland with extensive grassland areas (Jessen et al. 2015). In Pomerania (near Rusinowo) the woodlands from the early Preboreal are also relatively open, with remains of vegetation typical of the late Pleistocene: Artemisia, Chenopodiaceae or Rumex (Malkiewicz 2017). On the other hand, in western Pomerania (Germany) we note a rapid succession of birch-pine woodland, although with the presence of relicts of Younger Dryas flora (de Klerk 2004)—sadly we do not have enough data at our disposal as to define how open these woodlands were. The prevailing view is that during the Preboreal the climate of the mountain and piedmont zones was quite harsh, with the early Holocene warming reaching those areas of southern Poland with a certain delay (Baranowska and Malkiewicz 2003; Starkel et al. 2013). On the other hand, environmental data from studies around Lake Gościąż in central Poland point to relatively rapid environmental changes in the early Preboreal, involving rapid succession of thermophillic trees and plants (Ralska-Jasiewiczowa et al. 2003). In western Europe during the Preboreal, we note cooler and drier swings in climate which delayed the expansion of birch forests and which favoured the development of grassland communities (Bos et al. 2007; Hoek, Bos 2007). The most notable of such swings - known variously as the Piottino oscillation (Switzerland), the Friesland oscillation (Denmark), or the Rammelbeek phase (Netherlands) - is the so-called Preboreal oscillation (PBO), whose onset from profiles from the Netherlands is dated to c. 9480-9400 cal BC. It also transpires that local conditions may have affected environmental changes in the Preboreal, leading to local variations in biotopes. This phase is also visible in the profiles from Poland (De Klerk et al. 2007).

Nevertheless, already in 9550 cal BC at Lundby we note deposits of elk bones, with the oldest Mesolithic artefact-unheard of in the Final Palaeolithic-an elk antler mattock being dated at c. $9840 \mathrm{cal}$ BC (10,127 \pm 33 BP, AAR-15630) (Jessen et al. 2015, Table 2; Fig. 8a). This is evidence that the beginnings of Mesolithicstyle cultures appear at the very start of the Holocene, even if elsewhere in southern Scandinavia and western Europe their chronology may be younger.

And finally we have numerous pointers to some populations of Central European reindeer surviving the end of the Pleistocene and thriving in the woodland habitats of the early and middle Preboreal. This is evidenced not only by radiocarbondated reindeer remains from Stellmoor and Kartstein (Table 5) - as the reliability of these is questioned-but also, possibly, by antlers from Chmielewo in northeastern Poland, which are dated to $9870-9380 \mathrm{cal}$ BC with $94.5 \%$ probability, that is, to the Late Younger Dryas or early Preboreal (Giżejewski et al. 2004). The presence of 
Fig. 6 Reanalysis of the model of the Palaeolithic/Mesolithic boundary in the light of modelling radiocarbon dates from the North German Plain and the Eifel. a All dates concerned; b without the dates from Bedburg-Königshoven and Bützsee-Altfriesack; c without the dates from Bedburg-Königshoven, Bützsee-Altfriesack, Stellmoor and Kartstein

reindeer in western Europe at the onset of the Holocene is additionally confirmed by dates of bones from a specimen found at Rottenburg-Siebenlinden, namely 8570-8020 cal BC (9110 \pm 80 BP, ETH-8265) and 8560-8310 cal BC (9225 \pm 35 BP, GrA-39961), plus dates from southwestern England and Denmark (Drucker et al. 2011). Using radiocarbon dating on three Lyngby axes from Great Poland and northeastern Poland also produced dates from the early Preboreal. It does not seem likely that such objects would have been imported by Mesolithic hunters; we believe that these belong to the last reindeer hunters who, at the onset of the Holocene, continued to hunt a local reindeer population.

Summarizing the points made above, we may affirm that the environmental conditions of the early Preboreal favoured gradual cultural changes, in the wake of environmental change. For several hundred years the woodlands brought into existence by climatic warming were of quite an open character, with relatively numerous grassland communities typical of Late Pleistocene conditions. Hence such conditions were favourable for the continued existence of residual populations of reindeer.

\section{Ornamented Artefacts and Lithics}

In the Late and Final Palaeolithic the geometrical style of representation spreads across the broad expanses of northern Europe. From the onset of the Allerød, artefacts sporting patterns of densely engraved, parallel zigzag lines arranged in groups separated by unornamented surface become popular in this area (Płonka et al. 2011; Płonka and Kowalski 2017; Vang Petersen 2015). This decorative style may be encountered on a broad range of objects, such as: a stone plate, a horse mandible, a ceremonial object from elk antler, an antler rod, a red deer antler shaft, an amber elk figurine, and harpoons. Most of these artefacts, with the exception of harpoons, do not show any evidence of usewear, hence we may assume that they were primarily of symbolic importance in the life of Late and Final Palaeolithic hunter-gatherers. In the case of the ceremonial object from elk antler from Rusinowo, we determined that the rich geometrical ornamentation and anthropomorphic representation were the product of the combined labour of a group of people, and had most probably been conducted over a longer period (Diakowski and Płonka 2017). The depiction, an image of a woman with spread legs and emphasised vulva, was made in geometrical style conforming to the rest of the ornamentation (Fig. 7a). Polishing of the surface and the zigzag lines of the ornament points to the object's long-term use, up to the moment of its abandonment.

Ornamentation styles used by Late and Final Palaeolithic hunters are continued in the Maglemosian art from northwestern and Central Europe. The pattern of densely engraved zigzag lines and anthropomorphic representations in the geometrical style, 

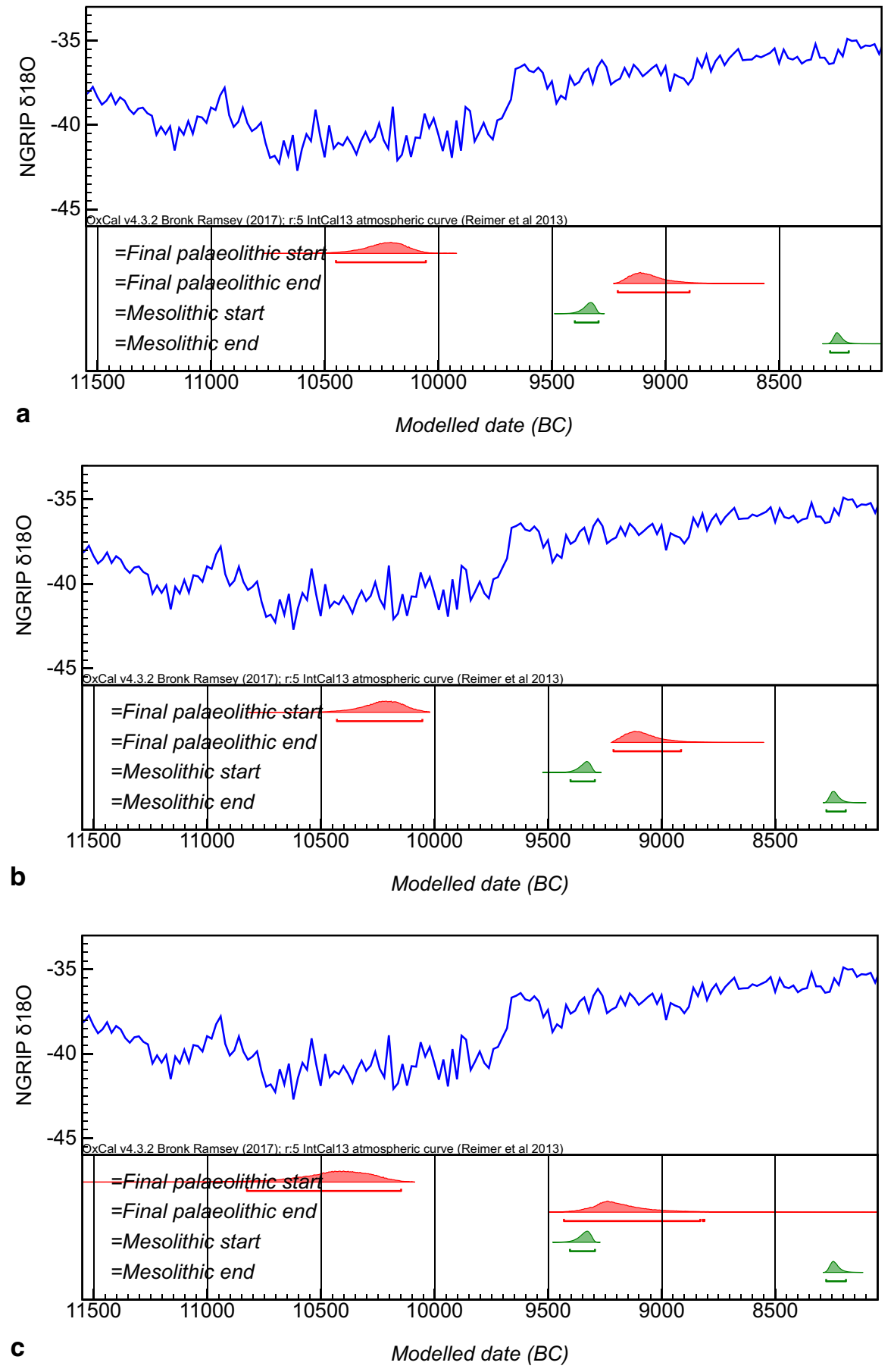
with human depictions composed of strokes are found on objects made from bone, antler and amber. Such finds come from various areas of early Mesolithic settlement: today's Denmark, northern Germany, England and northwestern Poland. There are striking stylistic similarities in anthropomorphic representation between the Late Palaeolithic artefact from Rusinowo and the bone fragment from Bolków, site 1 (Fig. 7a, b), the latter being found in the layers of an early Mesolithic site related to the complex of northwestern Mesolithic cultures (Galiński 2014, Fig. 13b). The distance between those two sites is $85 \mathrm{~km}$ in a straight line, while their ages are dated some 1900-2300 years apart. The simplest explanation for this would be a continuation of human settlement, and of the same cultural tradition, gradually changing over time. If we were to assume the migration and hiatus model, then Final Palaeolithic hunters would have to migrate to an area where the northwestern Mesolithic cultures milieu emerged, and then move back and resettle Pomerania again.

While the custom of decorating hunting weapons with simple geometric patterns is almost unheard of in the northwestern province of Mesolithic art, it is a highly characteristic feature of the Mesolithic in the eastern area of the Baltic Sea Basin and northeastern Europe (Płonka 2003). It is difficult to explain in simple terms how this borrowing came to be; it is not necessarily connected to human migration but might be connected to local traditions related to the Final Palaeolithic. The distribution of chocolate flint among Swiderian communities shows that single items regularly reached up to 300-400 km away from their outcrop of origin (Sulgostowska 1997a, 2005, 2008). A single site, Salaspils Laukskola near Riga, provides evidence for the existence of a distribution network reaching up to $600 \mathrm{~km}$ from the outcrop. Radiocarbon dates of biserially barbed harpoon heads found at Lake Lubanas in Latvia place them at the transition from the Pleistocene to the Holocene, or Late Preboreal, that is, 9760-9210 cal BC (Meadows et al. 2014; see also Ukkonen et al. 2006), thus confirming the continuation of Final Palaeolithic cultural forms in this area into the early Holocene.

An often-used argument in support of the theory of the emigration of Swiderian hunters to northeastern Europe is the lack of continuity between lithics forms used by this people and early Mesolithic flint working (Kozłowski and Kozłowski 1977; Kozłowski 1972; Więckowska 1975). Still, some authors note that in some Swiderian assemblages from central and eastern Poland we find forms of truncated and backed points which could be deemed to be the precursors of Mesolithic microliths. At some Final Palaeolithic sites, particularly in those in the middle Vistula basin, we find small truncations (Burdukiewicz 2011, p. 307). Here Więckowska (1975, p. 419) draws attention to Rydno II/59, while in this context Galiński (2002, pp. 340-341) mentions artefacts from Level VI, Trench V at Całowanie and inventories from Grochale Górne, which include, for example, Zonhoven points. The inventory from Krajkowo 1 (near Poznań) published not so long ago lists microliths and finds representative of the flint working style of the late Tanged Point Complexthe Ahrensburgian and Swiderian (Dmochowski and Dziewanowski 2008). Forms of this type (microlithic truncated points) are already known from older stages of the Younger Dryas, as evidenced by the inventory of Level V at Całowanie, Trench XI (Schild 1975, pp. 278-279), where a whole group of microliths of this type was found. Although this inventory is viewed as atypical, one must say that 

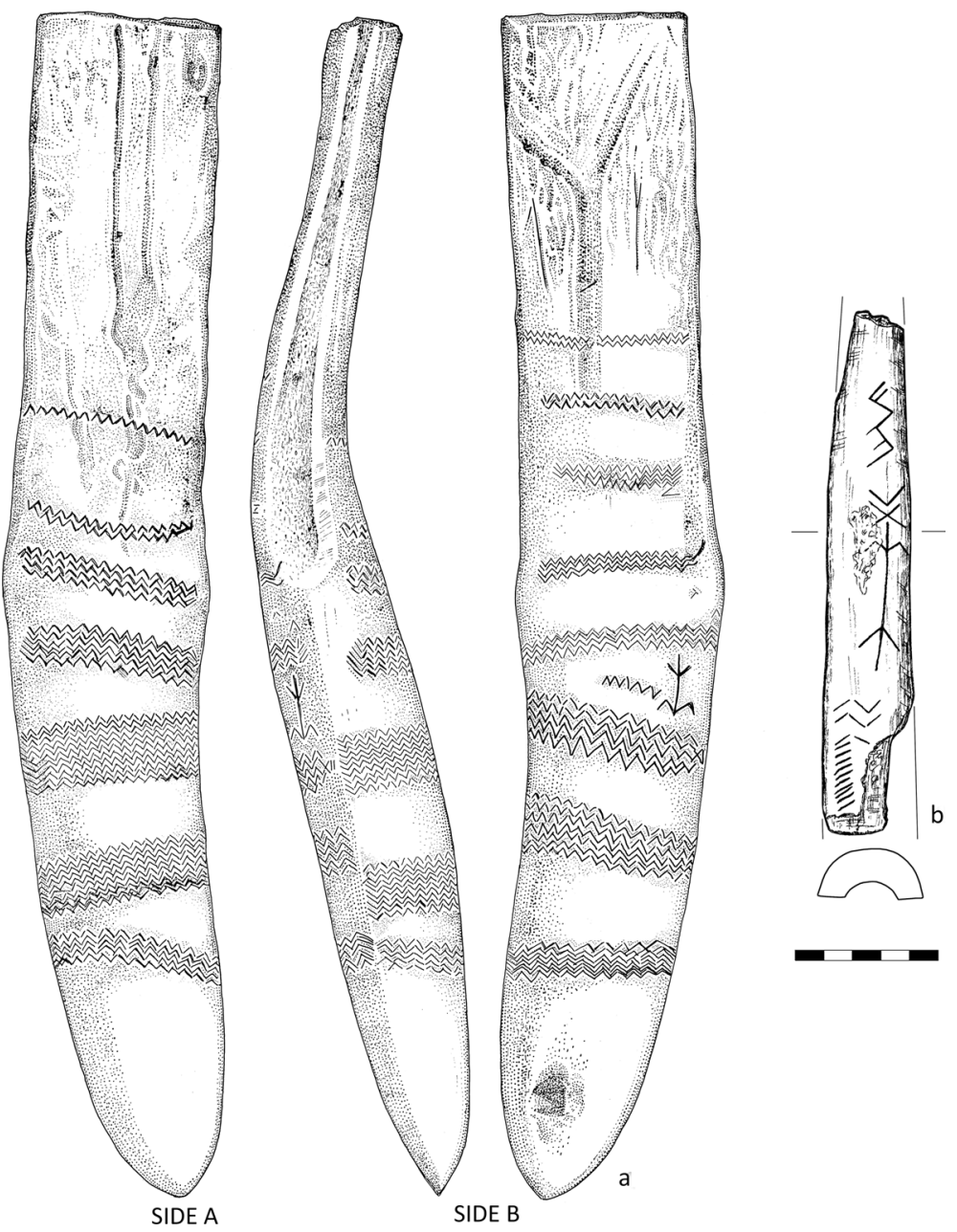

Fig. 7 Late Palaeolithic and early Mesolithic art from NW Poland (Pomerania). a Rusinowo; b Bolków 1

the manufacture of microlithic tools did not exceed the capability or skill of people representing Swiderian traditions. The tradition of microlith use is also visible at the Younger Dryas Swiderian campsite at Witów, central Poland. Besides classical Swiderian points, the Witów site also produced finds of microlithic truncated and Zonhoven points (Schild 1975, p. 282). Microlithic truncated points, including those of the Zonhoven type, are also known from other Swiderian sites (Schild 1975, p. 289).

A short time ago materials concerning concentration PF/2011 at Bolków, site 1 , in Pomerania were published; this concentration displays many features characteristic for Final Palaeolithic sites of the Epi-Ahrensburgian type (Galiński 2018). In this assemblage blanks were usually knapped from double platform cores with opposite platforms. The list of tools includes three tanged points-two 
Ahrensburgian and one Swiderian; two arch-backed points; and various forms of truncated points, including the Zonhoven type (Galiński 2018, Fig. 7). This site dates to the early and middle Preboreal (Table 1, Nos. 2 and 3).

To summarise the points made above, Swiderian hunters should not be perceived as incapable of modernisation of their hunting weapons. Microlithic forms have been present in the Swiderian assemblages from their inception-this is also true in the Ahrensburgian and Federmesser groups, as well as in Final Palaeolithic Epi-Ahrensburgian sites. Archaeological data from across the North European Plain show an increasing number of finds of microliths, truncated and backed points at sites from the end of the Younger Dryas and early Preboreal. One may assume that such forms were originally part of projectile points, and thus their presence would be linked with the spread of a new type of hunting weapon. Hence it transpires that in this case we are dealing with arrowheadswith the bow gaining immensely in importance at the end of the Pleistocene (Fischer 1989; Rozoy 1978, 1989; Serwatka 2018). Besides the point itself, mounted at the end of the shaft, the arrowhead also includes barbs-truncated and back points, most probably mounted in pairs to the sides of the shaft. Arrows with microlith barbs could have caused more severe wounds to the animal-their sharp edges cutting blood vessels, nerves and tendons, with devastating effects on an organism's vital parts, rendering them inactive. The spread of this invention was helped by the expansion of the birch, a tree type providing an inexhaustible supply of pitch used as adhesive to fix the microliths to the shaft. The use of pitch is confirmed by Mesolithic sites from northwestern Europe (Aveling and Heron 1999; Rozoy 1989; Sulgostowska 1997b). The changes in arrowhead design, just like any other effective weapons-related innovation, rapidly spread among communities of the European Plains.

It is difficult for us to explain the popularity of this innovation in hunting weapon design; everything points to many different factors being at play here, with environmental changes being just one. It could be linked to changes in perceptions of a weapon's effectiveness, as mentioned by Peter Bleed (1986, p. 739) who, in his seminal paper on weapon designs, wrote that besides being effective, a weapon must also 'meet social expectations'. Here effectiveness is understood as maximization of damage to the target, as caused by an arrow with a barbed head, assuming that the hunter aimed at the chest, that is, a large target containing various vital organs, damage to which caused the animal's death (Fischer 1989; Guthrie 2005, pp. 240-241). The second microlith on the side of the famous arrow from Loshult in southern Sweden is not a barb but a lateral cutting edge (Rozoy 1989, p. 18). Adding such cutting edges to arrows prevented them from slipping out of the wound. If the animal was not killed on the spot and bolted, it would keep on snagging the shaft against tree branches, weakening itself even further. However, although in a wooded environment an escaping animal is indeed more likely to hit an arrow protruding from its body against a branch and thus cause further damage to itself, it is difficult to decide whether this was a consideration in the popularization of this new weapon. Such a simple correlation should probably be ruled out, especially as microliths make their first appearance in the Younger Dryas, in almost treeless tundra conditions. 


\section{Social Circumstances and Migration}

As already mentioned, one of the arguments used in support of the theory that Polish lands were abandoned at the close of the Pleistocene, with a hiatus between the departure of the previous population and the arrival of Mesolithic settlers, is the demise of chocolate flint mining and the collapse of its distribution network, as well as the scant traces of settlement around the ochre mine in Rydno (Schild 1996a, 1996b, 2001). In this context it should be noted that explaining mechanisms of cultural change using coarse-grained archaeological evidence is a difficult and thankless task. Such changes are the outcome of a mix of circumstances, of factors difficult to identify and combine into some sort of structure, especially as only a negligible percentage of such factors leaves traces in the archaeological record. One example of difficult-to-interpret cultural behaviour is the model of exchange of shells and fossils which lasted in southwestern Germany from the Magdalenian to the Mesolithic (Eriksen 2002). We note the inflow of shell imports during the Magdalenian and in the Mesolithic_yet in the Final Palaeolithic such imports dwindle to almost nil, for no known reason, with no evidence of any change to the resident population. The very same mechanism might apply to early Mesolithic dwellers ceasing to extract ochre at Rydno. In exactly the same way as with flint, acquisition efforts may have been redirected into use of local ochre sources. Additionally, at Rydno there are signs of Komornica-culture settlement from the early Mesolithic-however, a large part of the site was destroyed before it was excavated (Schild et al. 2011, pp. 47, 331-344). The termination of exploitation of the chocolate flint outcrop by early Mesolithic dwellers may have been driven by technological considerations - the needs of early Mesolithic hunters-gatherers being fully met by surface-gathered erratic, chocolate or Jurassic flint. We find examples of use of erratic chocolate flint in the Final Palaeolithic/early Mesolithic at the aforementioned site no. 1 Krajkowo near Poznan. This flint may have come from a secondary source which could not, however, have been far removed from the original outcrops (Dmochowski and Dziewanowski 2008, p. 408). As an aside here, we should mention that pioneer communities do not seem to have had difficulty in finding essential stone resources: flint for tools and pigments. Magdalenian immigrants to southern Poland may serve as an example of this, as they quickly found their way around the available Late Pleistocene resources and exploited various local outcrops of flint and pigment (Kozłowski and Sachse-Kozłowska 1995). Therefore, even if Mesolithic communities were newcomers to this area they doubtlessly would have identified locally available resources. The trade networks for flint and pigments from the Late and Final Palaeolithic could not function as efficiently as previously due to changed conditions in the dense woodlands of the temperate zone. Furthermore, this was a period when we note the rise of local social relations as reflected in the diversification of stone inventories and art motifs (Kozłowski 2009; Kozłowski and Kozłowski 1975; Płonka 2003; Stoczkowski 1987). Hence the simple explanation could be that mined flint and ochre were simply not attractive enough to become trade items, with other prestige objects being of greater relevance.

Migration as a factor explaining cultural change at a time of dramatic environmental changes requires separate investigation. It is noteworthy that none of the 
quoted texts suggesting the depopulation of Polish lands in the Late Pleistocene provides a definition of migration, with this term being differently understood in studies of ecology or biology, geography, genetics, history or other sciences (Anthony 1990; Burmeister 2000; Clark 1994); neither has there been any discussion of the models and pace of migration movements, nor debate about the theoretical aspects of how migrations might be reflected in the archaeological record. This is in spite of the view expressed by David Anthony that using archaeological data to prove human migration is rather foolhardy (Anthony 1990, p. 95). Furthermore, in the archaeology of the Palaeolithic the use of migrations as an explanation of anything is frowned upon, due to the low population density of that period (Clark 1994). In the opinion of Graham Clark (1994, pp. 305-307) the terms migration and diffusion are more pertinent for the historical period, and using them as an explanation in the archaeology of the Palaeolithic is inappropriate. Such a position is, naturally, too extreme and reflects processual archaeology's dislike of using these phenomena in interpretation of cultural processes (Burmeister 2000).

According to Anthony, the most appropriate approach towards the issue of migration starts by defining its nature. In his opinion 'migration can be understood as a behaviour that is typically performed by defined subgroups (often kin-recruited) with specific goals, targeted on known destinations and likely to use familiar routes' (Anthony 1990, pp. 895-896). He identifies the main drivers as negative factors at the point of origin (push) and positive factors at the destination (pull) - although such an approach is criticized as being inflexible and ahistorical (Burmeister 2000). Although push factors are mostly economic in nature, religious, cultural or social (oppression) considerations are also possible. As concerns Younger Dryas inhabitants of the Polish Plain, the negative factor would have been the extinction or migration of cold-adapted Pleistocene species, reindeer in particular. However, the change in fauna was not as drastic as previously thought, with reindeer surviving into the oldest phases of the Preboreal and coexisting with woodland species. Hence environmental conditions allowed for gradual adaptation to the new situation, without the need to assume significant changes to the human population. Naturally, it cannot be ruled out that some communities gradually moved northwards following the new geographic range of reindeer, yet it is difficult to view this as an organised migration in the sense defined above.

In our opinion it would be best to look at the likelihood of human migration through a model showing its pace (dynamics) in relation to the predictability of the environment. The predictability may be defined as 'the degree to which a correct forecast [or prediction] of a system's state can be made either qualitatively or quantitatively' (Mihailović et al. 2016, p. 266). The assumption is that predictability of environment is high when the climate is stable and is lowered in conditions of rapid climate change. Such modelling looks at four extreme scenarios (Fig. 8):

1. high predictability and low rate of migration-a situation typical of stable environmental conditions and stable social relations; these are individuals and families, driven by personal considerations, who leave the group (Gamble 1998);

2. high predictability and high rate of migration - this scenario happens when migration is driven by non-economic factors: social, religious or cultural. In our opinion 
the migration of the Magdalenian population from southwest to Central Europe is an example of this model (Jochim et al. 1999; Svoboda et al. 1996). As the area of origin had stable environmental conditions, one may assume that the outbound migration was brought about by other than economic factors-probably an increase in population leading to problems with social organisation (Płonka 2012);

3. low predictability and low rate of migration - this occurs during times of rapid climate change; strong social cohesion will cushion the impact of change (Whallon 2006); archaeological data points to the existence of such strong social bonds in the Swiderian and Ahrensburgian in the Polish plains, as evidenced by trade networks for the exchange of raw materials (Sulgostowska 1997a, 2005, 2008);

4. low predictability and high rate of migration - this is when rapid environmental changes are accompanied by high migration rates; this is found in conditions of weak social ties and highly specialized cultures. To date this model has been used to explain the cultural changes in the Polish Plain at the Palaeolithic-Mesolithic transition.

Of course, there are many intermediate scenarios between these extreme examples which are, however, difficult to identify using coarse-grained archaeological evidence. Nevertheless, the existing archaeological record provides the largest quantity of supporting evidence for scenario no. 3. People from the Final Palaeolithic living in the Younger Dryas formed well-organized communities connected by a strong network of mutual relations. It is therefore unlikely that environmental changes would have triggered a migration, especially as some elements of the new environment, for instance, elk, were already known in Central Europe during the Younger Dryas (Płonka et al. 2011), whereas reindeer was still present in Polish lands in the Preboreal. It should also be noted that there are no signs whatsoever of human migration in the Central European Plain at the break of Allerød and Younger Dryas, a time of dramatic change in climate.

It may therefore be assumed that any human movements affecting the Polish Plain at the transition from Pleistocene to Holocene were not particularly dramatic in scope. In our view it would be better to explain such movements using the term dispersal-which, unlike migration, means expansion, but not as focused along a specific axis_and the category familiar area. 'A familiar area is the entire area known to a species, although not all parts of it will be known to individuals or would be regularly exploited by any single group or individual. The familiar area can be many times greater than a species' home range' (Clark 1994, pp. 313-314). A clear example of such familiarity is the above-mentioned site of Salaspils Laukskola near Riga, some $600 \mathrm{~km}$ away from the outcrop of chocolate flint which provided the raw material for a large part of the inventory.

To add an anthropological dimension we searched - using the tag migrationthe e-HRAF database for hunter-gatherer communities of the arctic and sub-arctic areas of North America and Asia. Naturally enough the term migration is used in explaining the mechanisms through which various areas of the Arctic and Far North were settled. Nevertheless, such processes behind human movements may involve quite distant times and are thus similar to our subject area-and in some 


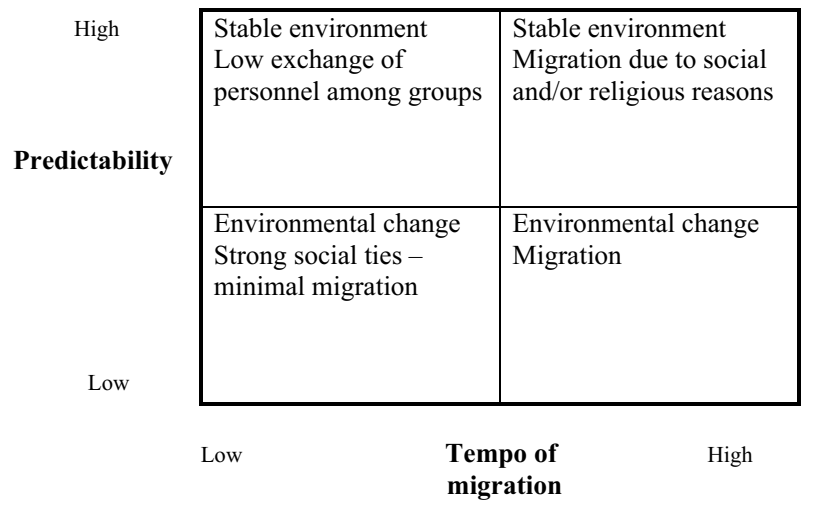

Fig. 8 Model presenting a tempo of migration vs. the predictability of the environment

cases migrations are questioned, specifically in relation to the Nenets, part of the Cree or Ojibwa. There are instances where a migration has partly moved into the realm of legend, becoming a Great Journey, a part of a tribe's religious life and the axis of its ideology, for example among the Ojibwa, where tradition holds that they abandoned their previous grounds near the mouth of the Saint Lawrence river and moved to the Great Lakes area (Bishop 1974; Greenberg and Morrison 1982).

Of greater interest is information about the reactions of various hunter-gatherer communities to the disappearance of their traditional prey-the reindeer. Sadly such scenarios are not well represented in the database. The Copper Inuit from Victoria Island in northern Canada had a problem when, after 1925, the spring and summer migrations of reindeer ceased (Damas 1972, pp. 17, 19). Different Inuit communities reacted in different ways. Some sought replacement for reindeer through hunting for seals or fishing either in the west of the island, around Prince Albert Sound, or on the eastern coast-around Wellington Bay and Cambridge Bay. However, some hunters, particularly from the eastern part of island, moved to the mainland, for instance to the Kent Peninsula, to continue their hunting for reindeer, still occasionally encountered in that area.

Another account concerns the Naskapi (Innu) who, up to the early $20^{\text {th }}$ century, sustained themselves with hunting forest reindeer around House Lake (Naskapi: Mushuan Nipi), in northeastern Canada. Around 1916, reindeer changed their migration routes, which caused a famine threatening the existence of the Naskapi (Henriksen 1973). This led the Naskapi to move to the coast and seek succour at Hudson Bay Company outposts. This disaster was brought about by the total dependence of the Naskapi on reindeer. They did not know how to hunt for sea animals, as previously they had visited the coast solely to trade. Tellingly they did not look for the reindeer's new migration routes to their northwest-probably their material culture was so tailored to their woodland habitat that they decided against hunting reindeer in the tundra, a treeless landscape totally alien to them (Tanner 1944, pp. 677-678). 
The situations described above, in spite of having happened in the period of globalization where people with traditional lifestyles live alongside complex Western societies, nevertheless shed light on the cultural mechanisms behind human migration and dispersion. The most salient conclusion seems to be that decisions-regardless of cultural and social motives - are most dependent upon the role of the 'staple prey' - in this case reindeer-in the affected population's diet and material culture. The Copper Inuit switched to different prey or partly employed periodical dispersion to adapt to their new situation. The Naskapi chose to migrate-albeit making use of an opportunity provided by the new, globalised world- to the trade outposts of white settlers. Now looking at the Polish and Central European Plain in the early Preboreal we see that it still harboured herds of reindeer-probably well adapted to the prevailing conditions of a woodland landscape with broad expanses of open terrain.

To sum up this theoretical examination of the process of human migration and review of the anthropological data, we must admit that the data do not point to migrations being common among hunter-gatherer populations; the movements of such communities are usually better described with the term dispersal. It is doubtful that Final Palaeolithic communities of the Polish Plain, bound together by strong social bonds, would not have been capable of adapting to new environmental conditions. Analysis of the chronology of the Pleistocene-Holocene transition suggests that change in that period is best described as a gradual process, allowing for parallel existence of the old, Final Palaeolithic and new, Mesolithic ways of life.

\section{Conclusion}

The available data-results of statistical modelling of radiocarbon dating, and data concerning the natural environment, symbolic culture and flint artefacts, and the information on hunter-gatherer social life-give grounds to question the theory that Polish lands were depopulated during the final stages of the Pleistocene or onset of the Holocene, and then repopulated by an inflow of Mesolithic settlers after a settlement hiatus. The analysed dates tend to point to Final Palaeolithic and Mesolithic settlement not being separated in chronological terms. The results of our research support what we term here the 'replacement model', and convince us that in the Polish Plain the Mesolithic directly succeeded the Final Palaeolithic some time between 9260 and $8980 B C$, with a very low probability of overlap between the Final Palaeolithic and Mesolithic settlement (11\%). It is difficult to use existing data to theorise the earlier emergence of the Mesolithic in Germany and western Poland. However, in light of the presented data it is difficult to advance the view that it was related to a gradual eastward migration of Mesolithic hunters, as there is no evidence for a mass exodus to the north by the Final Palaeolithic population.

A slightly different model, the 'coexistence model', regarding the disappearance of Final Palaeolithic traditions and the emergence of a new way of life may be assumed to apply to the North German Plain. Here we can argue that the two cultural patterns coexisted alongside one another for some two hundred years: there the Palaeolithic terminates between 9210 and 8900 BC, while the Mesolithic begins 
somewhere between 9400 and $9300 \mathrm{BC}$. Here the modelling of radiocarbon dating points to an overlap between Final Palaeolithic and Mesolithic settlement, where even the elimination of earliest Mesolithic dates from Bützsee-Altfriesack, BedburgKönigshoven and the latest Final Palaeolithic dates from Stellmoor or Kartstein does not fundamentally alter the results of the modelling; this change merely lowers the probability of the conclusion.

In eastern Poland the Mesolithic began slightly later than in western Poland or Germany, possibly due to climate factors at the onset of the Preboreal period. Data from radiocarbon dating point to there being neither mass migrations nor a settlement hiatus. Environmental data, flint artefact forms and symbolic culture all confirm that human communities inhabiting the European Plain developed local cultural forms typical of the Mesolithic. Anthropological studies also add weight to the argument that a mass migration of Palaeolithic and Mesolithic hunter-gatherers was not very likely.

Although the corpus of analysed dates is rather limited, considering that they cover some two thousand years, it is nevertheless possible to theorise on the social phenomena behind the presented models of changes occurring in the Polish and North German Plains at the Palaeolithic-Mesolithic transition. The replacement model assumes a relatively rapid disappearance of cultural forms typical of the Final Palaeolithic and the emergence of Mesolithic forms. It is difficult to pinpoint the causes of this turn of events. However, one may theorise that it could stem from the presence of strong social bonds, from the rapid exchange of information between various human groups, with such an information exchange being accompanied by the dispersion to the northeast of those who preferred the traditional way of life. However, this never took on the character of a mass migration, but was rather a relocation inside a well-known landscape. It is likely that the coexistence model reflects a different set of social circumstances. Here we would have a highly diverse cultural landscape, where different niches are inhabited by different human groups-some cultivating old traditions, others following a new way of life. The existence of social interaction between these distinct communities may be surmised from the appearance of new flintworking techniques, as evidenced by, for example, Epi-Ahrensburgian assemblages. The question remains whether it is possible to interpret the models resulting from Bayesian analysis of existing radiocarbon dates. It seems that we still do not possess enough radiocarbon dates to allow for more precise analysis of transition-period processes in a regional perspective.

In light of the above analysis, the hypothesised settlement hiatus between the Final Palaeolithic and early Mesolithic, and mass early Holocene immigration into the Polish Plain appears to be rather unlikely. Such a theory is contradicted not only by the results of radiocarbon dating modelling, but also by data concerning the state of transformation of the habitat, artefacts from various materials, and remains of symbolic culture. The migration and hiatus theory is further undermined by results of analysis of anthropological materials concerning migrations of hunter-gatherer communities. During the Younger Dryas and at the beginning of the Preboreal the broad expanses of the North European Plain witness a change in the tool kit-the emergence and growing role of microlithic tools. In the early Preboreal there still exist communities continuing Late Pleistocene traditions. The migration of new 
communities of flora and fauna occurred in parallel to the existence of enclaves of grassland communities and fauna typical of the late Pleistocene. We conclude that during the Pleistocene/Holocene transition, the European Plain was host to complicated changes which cannot be explained by a simple linear evolutionary model. The cultural landscape was a patchwork, interweaving old forms with emerging new traits, the latter becoming broadly understood as early Mesolithic traditions.

Acknowledgements We wish to express our gratitude to R. Benecke, D. Holst, I. Sobkowiak-Tabaka, T. Terberger and D. Werra for their assistance in gaining access to publications not available at Polish libraries, as well as Janet Rethemeyer for information pertaining to radiocarbon dating at the laboratory at Köln. We also thank the reviewers for their remarks which helped us to improve our paper.

\section{Compliance with Ethical Standards}

Conflict of interest The authors declare that they have no conflict of interest.

Open Access This article is licensed under a Creative Commons Attribution 4.0 International License, which permits use, sharing, adaptation, distribution and reproduction in any medium or format, as long as you give appropriate credit to the original author(s) and the source, provide a link to the Creative Commons licence, and indicate if changes were made. The images or other third party material in this article are included in the article's Creative Commons licence, unless indicated otherwise in a credit line to the material. If material is not included in the article's Creative Commons licence and your intended use is not permitted by statutory regulation or exceeds the permitted use, you will need to obtain permission directly from the copyright holder. To view a copy of this licence, visit http://creativecommons.org/licen ses/by/4.0/.

\section{References}

Alberti, G. (2016). 'Prob.phases.relat': Posterior probability for different chronological relations between two Bayesian radiocarbon defined phases. https://doi.org/10.13140/rg.2.1.3713.2560.

Anthony, D. W. (1990). Migration in archeology: The baby and the bathwater. American Anthropologist (NS), 92(4), 895-914.

Aveling, E. M., \& Heron, C. (1999). Identification of birch bark tar at the Mesolithic site of Star Carr. Ancient Biomolecules, 2, 69-80.

Baales, M., Heinen, M., Orschiedt, J., Pollmann, H.-O., \& Stapel, B. (2015). Between North, West and South: Westphalian Mesolithic research during the last decade. In S. Sázelová, M. Novák, \& A. Mizerová (Eds.), Forgotten times and spaces: New perspectives in paleoanthropological, paleoetnological and archeological studies (pp. 448-467). Brno: Institute of Archeology of the Czech Academy of Sciences, Masaryk University.

Baranowska, A., \& Malkiewicz, M. (2003). Badania palinologiczne torfowisk holoceńskich na Dolnym Śląsku. Botanical Guidebooks, 26, 233-248.

Barton, R. N. E. (1998). Long blade technology and the question of British Late Pleistocene/Early Holocene lithic assemblages. In N. M. Ashton, F. Healy, \& P. Pettit (Eds.), Stone Age archaeology: Essays in honour of John Wymer (pp. 158-164). Oxford: Oxbow Books.

Benecke, N., Gramsch, B., \& Weisse, R. (2002). Zur Neudatierung des Ur-Fundes von Potsdam-Schlaatz, Brandenburg. Archäologisches Korrespondenzblatt, 32(2), 161-168.

Bishop, C. A. (1974). Northern Ojibwa and the fur trade: An historical and ecological study. Cultures and communities, a series of monographs: Native peoples. Retrieved December 30, 2018, from http://ehrafworldcultures.yale.edu/document?id=ng06-054.

Bittmann, F., \& Pasda, C. (1999). Die Entwicklung einer Düne während der letzten 12000 Jahre Untersuchungsergebnisse von Groß Lieskow (Stadt Cottbus) in der Niederlausitz. Quartär, 49(50), 39-54. 
Björck, S., Walker, M. J. C., Cwynar, L. C., Johnsen, S., Knudsen, K.-L., Lowe, J. J., et al. (1998). An event stratigraphy for the Last Termination in the North Atlantic region based on the Greenland ice-core record: A proposal by the INTIMATE group. Journal of Quaternary Science, 13(4), 283-292.

Bos, J. A. A., van Geel, B., van der Plicht, J., \& Bohncke, S. J. P. (2007). Preboreal climate oscillations in Europe: Wiggle-match dating and synthesis of Dutch high-resolution multi-proxy records. Quaternary Science Reviews, 26(15-16), 1927-1950.

Bleed, P. (1986). The optimal design of hunting weapons: Maintainability or reliability. American Antiquity, 51(4), 737-747.

Blockley, S. P. E., Lowe, J. J., Walker, M. J. C., Asioli, A., Trincardi, F., Coope, G. R., et al. (2004). Bayesian analysis of radiocarbon chronologies: Examples from the European Late-glacial. Journal of Quaternary Science, 19(2), 159-175.

Bokelmann, K. (1991). Duvensee, Wohnplatz 9. Ein präborealzeitlicher Lagerplatz in Schleswig-Holstein. Offa, 48, 75-114.

Bokelmann, K. (2012). Spade paddling on a Mesolithic lake: Remarks on Preboreal and Boreal sites from Duvensee (Northern Germany). In M. J. L. T. Niekus, R. N. E. Barton, M. Street et al. (Eds.), A mind set on flint. Studies in honour of Dick Stapert, part III: The Mesolithic and Neolithic (pp. 369-380). Groningen: Groningen Archaeological Studies.

Bokelmann, K., Averdieck, F.-R., \& Willkomm, H. (1982). Duvensee, Wohnplatz 8. Neue Aspekte zur Sammelwirtschaft im frühen Mesolithikum. Offa, 38(1981), 21-40.

Brace, S., Diekmann, Y., Booth, T. J., Faltyskova, Z., Rohland, N., Mallick, S., et al. (2019). Ancient genomes indicate population replacement in Early Neolithic Britain. Nature Ecology \& Evolution, 3, 765-771. https://doi.org/10.1038/s41559-019-0871-9.

Brinch Petersen, E. (2009). The human settlement of southern Scandinavia 12 500-8 700 cal BC. In M. Street, N. Barton, \& T. Terberger (Eds.), Humans, environment and chronology of the Late Glacial of the North European Plain. Proceedings of 14 Workshop of the 15th U.I.S.P.P. Congress, Lisbon, September 2006 (pp. 89-129). Mainz: Verlag des Römisch-Germanischen Zentralmuseums (RGZM-Tagungen 6).

Bronk Ramsey, C. (1995). Radiocarbon calibration and analysis of stratigraphy: The OxCal program. Radiocarbon, 37(2), 425-430.

Bronk Ramsey, C. (2009a). Bayesian analysis of radiocarbon dates. Radiocarbon, 51(1), 337-360.

Bronk Ramsey, C. (2009b). Dealing with outliers and offsets in radiocarbon dating. Radiocarbon, 51(3), 1023-1045.

Bronk Ramsey, C., Buck, C. E., Manning, S. W., Reimer, P., \& van der Plicht, H. (2006). Developments in radiocarbon calibration for archaeology. Antiquity, 80(310), 783-798.

Bronk Ramsey, C., Dee, M., Lee, S., Nakagawa, T., \& Staff, R. (2010). Developments in the calibration and modelling of radiocarbon dates. Radiocarbon, 52(3), 953-961.

Bronk Ramsey, C., Higham, T. F. G., Owen, D. C., Pike, A. W. G., \& Hedges, R. E. M. (2002). Radiocarbon dates from the Oxford AMS system: Archaeometry datelist 31. Archaeometry, 44, supplement s1, 1-150.

Bronk Ramsey, C., \& Lee, S. (2013). Recent and planned developments of the Program OxCal. Radiocarbon, 55(2-3), 720-730.

Burdukiewicz, J. M. (1999). Z problematyki paleolitu Sudetów. In P. Valde-Nowak (Ed.), Poczatki osadnictwa w Sudetach (pp. 35-52). Kraków: Instytut Archeologii i Etnologii PAN.

Burdukiewicz, J. M. (2011). Late Glacial hunter-gatherer reactions to the Younger Dryas cooling event in the southern and eastern Baltic regions of Europe. Quaternary International, 242, 302-312.

Burdukiewicz, J. M., \& Bronowiecki, J. (1999). Grupy myśliwsko-zbierackie w Sudetach w plejstocenie i wczesnym holocenie. In P. Valde-Nowak (Ed.), Poczatki osadnictwa w Sudetach (pp. 171-183). Kraków: Instytut Archeologii i Etnologii PAN.

Burmeister, S. (2000). Archaeology and migration: Approaches to an archaeological proof of migration. Current Anthropology, 41(4), 539-569.

Clark, G. A. (1994). Migration as an explanatory concept in Paleolithic archaeology. Journal of Archaeological Method and Theory, 1(4), 305-343.

Conneller, C. J., Bayliss, A., Milner, N., \& Taylor, B. (2016). The resettlement of the British landscape: Towards a chronology of Early Mesolithic lithic assemblage types. Internet Archaeology. https:// doi.org/10.11141/ia.42.12.

Crombé, P., Van Strydonck, M., \& Boudin, M. (2009). Towards a refinement of the absolute (typo) chronology for the early Mesolithic in the coversand area of northern Belgium and the southern 
Netherlands. In P. Crombé, M. Van Strydonck, J. Sergant, M. Bats, \& M. Boudin (Eds.), Chronology and evolution within the Mesolithic of north-west Europe: Proceedings of an international meeting, Brussels, May 30th-June 1st 2007 (pp. 95-112). Newcastle-upon-Tyne: Cambridge Scholars Publishing.

Cyrek, K. (1996). Osadnictwo schytkowopaleolityczne w Zakolu Załęczańskim doliny Warty. Biblioteka Muzeum Archeologicznego i Etnograficznego w Łodzi 30. Łódź: Muzeum Archeologiczne i Etnograficzne.

Cziesla, E., \& Pettitt, P. (2003). AMS-14C-Datierungen von spätpaläolitischen und mesolitischen Funden aus dem Bützsee (Brandenburg). Archäologisches Korrespondenzblatt, 33(1), 21-38.

Damas, D. (1972). Copper Eskimo. In M. G. Bicchieri (Ed.), Hunters and gatherers today: A socioeconomic study of eleven such cultures in the twentieth century (pp. 3-50). New York: Holt, Rinehart \& Winston. Retrieved December 29, 2018, from http://ehrafworldcultures.yale.edu/docum ent?id=nd08-032.

De Klerk, P. (2004). Changes in vegetation and environment at the Lateglacial-Holocene transition in Vorpommern (Northeast Germany). In T. Terberger, \& B. V. Eriksen (Eds.), Hunters in a changing world: Environment and archaeology of the Pleistocene-Holocene transition (ca. 11000-9000 $B C$ ) in northern central Europe. Internationale Archäologie-Arbeitsgemeinschaft. Tagung Symposium, Kongress 5 (pp. 27-42). Rahden: Verlag Marie Leidorf GmbH.

De Klerk, P., Couwenberg, J., \& Joosten, H. (2007). Short-lived vegetational and environmental change during the Preboreal in the Biebrza Upper Basin (NE Poland). Quaternary Science Reviews, 26(15-16), 1975-1988.

Diakowski, M., \& Płonka, T. (2017) Execution of the ornament. In T. Płonka, \& K. Kowalski (Eds.), Rusinowo. The symbolic culture of foragers in the Late Palaeolithic and the early Mesolithic (pp. 71-116). Wrocław: University of Wrocław Institute of Archaeology.

Dmochowski, P., \& Dziewanowski, M. (2008). Krajkowo 1: Unikatowy inwentarz wczesnego mezolitu wykonany z krzemienia czekoladowego w samym 'sercu' Wielkopolski. In M. Borkowski, J. Libera, B. Sałacińska, \& S. Sałaciński (Eds.), Krzemień czekoladowy w pradziejach. Materiały $z$ konferencji w Orońsku, 08-10.10.2003 (pp. 407-418). Warszawa-Lublin: Studia nad Gospodarką Surowcami Krzemiennymi w Pradziejach 7.

Drucker, D. G., Kind, C.-J., \& Stephan, E. (2011). Chronological and ecological information on Late-glacial and early Holocene reindeer from northwest Europe using radiocarbon $\left({ }^{14} \mathrm{C}\right)$ and stable isotope $\left({ }^{13} \mathrm{C},{ }^{15} \mathrm{~N}\right)$ analysis of bone collagen: Case study in southwestern Germany. Quaternary International, 245, 218-224.

Eriksen, B. V. (2002). Fossil mollusks end exotic raw materials in Late Glacial and early postglacial find contexts: A complement to lithic studies. In L. E. Fisher, \& B. V. Eriksen (Eds.), Lithic raw material economies in Late Glacial and Early Postglacial Europe (pp. 27-52). BAR Int. Series 1093. Oxford: Archaeopress.

Fiedorczuk, J. (1995). Mesolithic finds at Dudka, Great Masurian Lakeland, and their chronological-taxonomic relations. Przeglad Archeologiczny, 43, 47-59.

Fischer, A. (1989). Hunting with flint-tipped arrows: Results and experiences from practical experiments. In C. Bonsall (Ed.), The Mesolithic in Europe: Papers presented at the Third International Symposium, Edinburgh 1985 (pp. 29-39). Edinburgh: John Donald Publishers Ltd.

Fischer, A., \& Tauber, H. (1986). New C-14 datings of Late Palaeolithic cultures from northwestern Europe. Journal of Danish Archaeology, 5, 7-13.

Galiński, T. (1992). Mezolit Pomorza. Szczecin: Muzeum Narodowe w Szczecinie.

Galiński, T. (1997). Mezolit Europy. Szczecin: Drukarnia ZAPOL.

Galiński, T. (2002). Społeczeństwa mezolityczne: Osadnictwo, gospodarka, kultura ludów łowieckich w VIII-IV tysiacleciu p.n.e. na terenie Europy. Biblioteka Naukowa Muzeum Narodowego w Szczecinie. Seria Archeologiczna. Szczecin: Muzeum Narodowe w Szczecinie.

Galiński, T. (2014). Obozowiska łowieckie ze schyłku preborealu w Bolkowie na Pomorzu Zachodnim. Archeologia Polski, 59(1-2), 79-120.

Galiński, T. (2015a). Bolków nad jeziorem Świdwie: Nowe materiały kultury ahrensburskiej. Materiaty Zachodniopomorskie NS, 10(2013), 7-114.

Galiński, T. (2015b). Tanowo: Obozowiska łowców epoki paleolitu i mezolitu na Pomorzu. Warszawa: Instytut Archeologii i Etnologii Polskiej Akademii Nauk.

Galiński, T. (2015c). Najstarsze osadnictwo mezolityczne w strefie zachodniobałtyckiej w świetle badań stanowiska Bolków 1. Przeglad Archeologiczny, 63, 29-57. 
Galiński, T. (2018). Epiahrensburgian: Osadnictwo paleolityczne w początkach holocenu na stanowisku w Bolkowie na Pomorzu Zachodnim. Przeglad Archeologiczny, 66, 5-30.

Gamble, C. (1998). Palaeolithic society and the release from proximity: A network approach to intimate relations. World Archaeology, 29(3), 426-449.

Ginter, B. (1973). Remarks on the origin of some Mesolithic cultures in Poland. In S. K. Kozłowski (Ed.), The Mesolithic in Europe (pp. 177-186). Warsaw: University Press.

Giżejewski, Z., Wojtal, P., Csapó, J., Giżejewski, J., Jaczewski, Z., \& Csapó, J., Jr. (2004). Remains of Late Pleistocene reindeer from Chmielewo, northeast Poland. European Journal of Wildlife Research, 50(4), 207-212.

Gob, A. (1991). The early postglacial occupation of the southern part of the North Sea Basin. In N. Barton, A. J. Roberts, \& D. A. Roe (Eds.), The Late Glacial in north-west Europe (pp. 227-233). Oxford: Alden Press.

Goslar, T., Kabaciński, J., Makowiecki, D., Prinke, D., \& Winiarska-Kabacińska, M. (2006). Datowanie radiowęglowe zabytków z Kolekcji Epoki Kamienia Muzeum Archeologicznego w Poznaniu. Fontes Archaeologici Posnanienses, 42, 5-25.

Gramsch, B. (1973). Das Mesolithikum im Flachland zwischen Elbe und Oder, Part 1. Veröffentlichungen des Museums für Ur- und Frühgeschichte 7. Berlin: VEB Deutscher Verlag der Wissenschaften.

Gramsch, B. (1987). Zeugnisse menschlichen Aktivitäten in Verbindung mit dem spätglazialzeitlichen Ur-Fund am Schlaatz bei Potsdam. Veröffentlichungen des Museums für Ur- und Frühgeschichte Potsdam, 21, 47-51.

Gramsch, B. (2012). Mesolitische Knochenartefakte von Friesack, Fundplatz 4. Lkr. Havelland. Veröffentlichungen zur brandenburgischen Landesarchäologie, 45(2011), 7-59.

Gramsch, B. (2016). Friesack 4: Eine Feuchtbodenstation des Mesolithikums in Norddeutschland. In R. Benecke (Ed.), Subsistenz und Umwelt der Feuchtbodenstation Friesack 4 im Havelland: Ergebnisse der naturwissenschaftlichen Untersuchungen (pp. 9-24). Arbeitsberichte zur Bodendenkmalpflege in Brandenburg 29. Wünsdorf: Brandenburgisches Landesamt für Denkmalpflege und Archäologisches Landesmuseum.

Gramsch, B., \& Beran, J. (2010). Spätaltsteinzeitliche Funde von Wustermark, Fundplatz 22, Lkr. Havelland. Veröffentlichungen zur brandenburgischen Landesarchäologie, 41/42(2007), 95-142.

Greenberg, A. M., \& Morrison, J. (1982). Group identities in the boreal forest: The origin of the northern Ojibwa. Ethnohistory, 29(2), 75-102. Retrieved December 30, 2018, from http://ehrafworldcultu res.yale.edu/document?id=ng06-057.

Groß, D. (2014). Welt und Umwelt frühmesolithischer Jäger und Sammler: Mensch-Umwelt-Interaktion im Frühholozän in der nordmitteleuropäischen Tiefebene. Archäologische Informationen, 37, 213-224.

Groß, D., Lübke, H., Meadows, J., Jantzen, D., \& Dreibrodt, S. (2019). Re-evaluation of the site Hohen Viecheln 1. In D. Groß., H. Lübke, J. Meadows, \& D. Jantzen (Eds.), From bone and antler to Early Mesolithic in northern Europe (pp. 15-111). Untersuchungen und Materialien zur Steinzeit in Schleswig-Holstein und im Ostseeraum 10. Kiel/Hamburg: K. Wachholtz Verlag. Retrieved November 22, 2019, from https://www.wachholtz-verlag.de/out/media_rte/Open\%20Access\%20 TEST/Gross_et_al_Working_Sharp_End_Steinzeit_Ostseeraum_10_978-3-529-01861-9_Gross _HohenViecheln.pdf.

Grünberg, J. M. (2016). The Mesolithic burials of the Middle Elbe-Saale region. In J. M. Grünberg, B. Gramsch, L. Larsson, J. Orschiedt, \& H. Meller (Eds.), Mesolithic burials: Rites, symbols and social organisation of early postglacial communities. International Conference, Halle (Saale), Germany 18th-21st September 2013 (pp. 257-290). Tagungen des Landesmuseums für Vorgeschichte Hallle 13/1. Halle (Saale): Landesamt für Denkmalpflege und Archäologie SachsenAnhalt/Landesmuseum für Vorgeschichte.

Gumiński, W. (1995). Environment, economy and habitation during the Mesolithic at Dudka, Great Masurian Lakeland, NE Poland. Przeglad Archeologiczny, 43, 5-46.

Günther, T., Malmström, H., Svensson, E. M., Omrak, A., Sánchez-Quinto, F., Kılınç, G. M., Krzewińska, M., Eriksson, G., Fraser, M., Edlund, H., Munters, A. R., Coutinho, A., Simões, L. G., Vicente, M., Sjölander, A., Jansen Sellevold, B., Jørgensen, R., Claes, P., Shriver, M. D., Valdiosera, C., Netea, M. G., Apel, J., Lidén, K., Skar, B., Storå, J., Götherström, A., \& Jakobsson, M. (2018). Population genomics of Mesolithic Scandinavia: Investigating early postglacial migration routes and highlatitude adaptation. PLoS Biology, 16(1):e2003703. Retrieved November 27, 1919, from https:// doi.org/10.1371/journal.pbio.2003703.

Guthrie, R. D. (2005). The nature of Paleolithic art. Chicago: University of Chicago Press. 
Heinen, M. (2014). Waldjäger: Das Mesolithikum im Rheinland. In L. Giemsch \& R. W. Schmitz (Eds.), Eiszeitjäger-Leben im Paradies. Europa vor 15000 Jahren. (pp. 289-311). Bonn: LVR-Landesmuseum.

Henriksen, G. (1973). Hunters in the Barrens: The Naskapi on the edge of the white man's world. Newfoundland Social and Economic Studies 12. St. John's: Institute of Social and Economic Research, Memorial University of Newfoundland. Retrieved December 29, 2018, from http://ehrafworldcultu res.yale.edu/document?id=nh06-019.

Hoek, W. Z., \& Bos, J. A. A. (2007). Early Holocene climate oscillations: Causes and consequences. Quaternary Science Reviews, 26, 1901-1906.

Jessen, C. A., Pedersen, K. B., Christensen, C., Olsen, J., Mortensen, M. F., \& Hansen, K. M. (2015). Early Maglemosian culture in the Preboreal landscape: Archaeology and vegetation from the earliest Mesolithic site in Denmark at Lundby Mose, Sjælland. Quaternary International, 378, 73-87.

Jochim, M., Herhahn, C., \& Starr, H. (1999). The Magdalenian colonization of southern Germany. American Anthropologist, 101(1), 129-142.

Johansen, L., \& Stapert, D. (2000). Two Epi-Ahrensburgian sites in the northern Netherlands: Oudehaske (Friesland) and Gramsbergen (Overijssel). Palaeohistoria, 39-40(1997-1998), 1-88.

Kabaciński, J. (2016). After the Ice Age. In J. Kabaciński (Ed.), The past societies: Polish lands from the first evidence of human presence to the early Middle Ages. Vol. 1: 500,000-5500 BC (pp. 249270). Warszawa: Institute of Archaeology and Ethnology, Polish Academy of Sciences.

Kabaciński, J., \& Sobkowiak-Tabaka, I. (2010). Środowiskowe uwarunkowania przemian kulturowych u schyłku późnego glacjału i w początkach holocenu na Niżu Europejskim. Przeglad Archeologiczny, 58, 5-21.

Kitagawa, H., \& van der Plicht, J. (1998). Atmospheric radiocarbon calibration to 45,000 yr BP: Late Glacial fluctuations and cosmogenic isotope production. Science, 279, 1187-1190.

Kobusiewicz, M. (1999). Ludy towiecko-zbierackie północno-zachodniej Polski. Prace Komisji Archeologicznej 19. Poznań: Poznańskie Towarzystwo Przyjaciół Nauk, Wydział Historii i Nauk Społecznych.

Kobusiewicz, M. (2004). The problem of the Palaeolithic-Mesolithic transition on the Polish Plain: State of research. In T. Terberger \& B. V. Eriksen (Eds.), Hunters in a changing world: Environment and archaeology of the Pleistocen-Holocene Transition (ca. 11000-9000 BC) in northern central Europe. Internationale Archäologie-Arbeitsgemeinschaft (pp. 133-139). Tagung Symposium, Kongress 5. Rahden: Verlag Marie Leidorf GmbH.

Kobusiewicz, M. (2016). Wojnowo, stanowisko 'a'. In M. Kobusiewicz (Ed.), Region Wojnowo: Arkadia towców i zbieraczy (pp. 117-183). Poznań: Ośrodek Studiów Pradziejowych i Średniowiecznych Instytut Archeologii i Etnologii Polskiej Akademii Nauk.

Kobusiewicz, M., Bobrowski, P., \& Jórdeczka, M. (2016). Wojnowo, stanowisko 4. In M. Kobusiewicz (Ed.), Region Wojnowo: Arkadia łowców i zbieraczy (pp. 251-281). Poznań: Ośrodek Studiów Pradziejowych i Średniowiecznych Instytut Archeologii i Etnologii Polskiej Akademii Nauk.

Kobusiewicz, M., \& Bower, J. (2016). Wojnowo, stanowisko 2. In M. Kobusiewicz (Ed.), Region Wojnowo: Arkadia łowców i zbieraczy (pp. 199-231). Poznań: Ośrodek Studiów Pradziejowych i Średniowiecznych Instytut Archeologii i Etnologii Polskiej Akademii Nauk.

Kobusiewicz, M., \& Kabaciński, J. (1993). Chwalim: Subboreal hunters-gatherers of the Polish Plain. Poznań: Institute of Archaeology and Ethnology Polish Academy of Sciences.

Kondracki, J. (1998). Geografia regionalna Polski. Warszawa: Wydawnictwo Naukowe PWN.

Kostrzewski, J. (1939). Od mezolitu do okresu wędrówek ludów. In Prehistoria ziem polskich. Encyklopedia polska, vol. IV, part 1-Branch V (pp. 118-359). Kraków: Polska Akademia Umiejętności.

Kozarski, S., Nowaczyk, B., Rotnicki, K., \& Tobolski, K. (1969). The eolian phenomena in west-central Poland with special reference to the chronology of phases of eolian activity. Geographia Polonica, 17, 231-248.

Kozłowski, J. K. (2001). La recolonisation tardiglaciaire et les changements culturels à la limite Pléistocène-Holocène sur la Grande Plaine. In Préhistoire de la Grande Plaine du Nord de l'Europe, Actes du Colloque Chaire Francqui interuniversitaire au titre étranger (Université de Liège, 26 juin 2001) (pp. 115-127). Liège: ERAUL 99.

Kozłowski, J. K., \& Kaczanowski, P. (2003). Najdawniejsze dzieje ziem polskich. Wielka Historia Polski (Vol. 1). Kraków: Fogra.

Kozłowski, J. K., \& Kozłowski, S. K. (1975). Pradzieje Europy od XL do IV tysiaclecia p.n.e. Warszawa: Państwowe Wydawnictwo Naukowe. 
Kozłowski, J. K., \& Kozłowski, S. K. (1977). Epoka kamienia na ziemiach polskich. Warszawa: Państwowe Wydawnictwo Naukowe.

Kozłowski, S. K. (1969). Z problematyki polskiego mezolitu (cz. 9): W sprawie granicy paleolitu i mezolitu w Polsce. Światowit, 30, 117-134.

Kozłowski, S. K. (1972). Pradzieje ziem polskich od IX do V tysiqclecia p.n.e. Warszawa: Państwowe Wydawnictwo Naukowe.

Kozłowski, S. K. (1989). Mesolithic in Poland: A new approach. Warsaw: University Press.

Kozłowski, S. K. (2009). Thinking mesolithic. Oxford: Oxbow Books.

Kozłowski, S. K., \& Sachse-Kozłowska, E. (Eds.). (1995). Maszycka Cave: A Magdalenian site in southern Poland. Jahrbuch des Römisch-Germanischen Zentralmuseums Mainz, 40(1) (1993), 115-252.

Lowe, J. J., Rasmussen, S. O., Björck, S., Hoek, W. Z., Steffensen, J. P., Walker, M. J. C., et al. (2008). Synchronisation of palaeoenvironmental events in the North Atlantic region during the Last Termination: A revised protocol recommended by the INTIMATE group. Quaternary Science Reviews, 27, 6-17.

Majchrowska, A., \& Popińska, E. (2018). Weryfikacja i uszczegółowienie granic mezoregionów fizycznogeograficznych województwa łódzkiego na podstawie współczesnych danych przestrzennych. Prace i Studia Geograficzne, 63(1), 93-107.

Malkiewicz, M. (2017). The vegetation in the light of palynological studies. In T. Płonka \& K. Kowalski (Eds.), Rusinowo: The symbolic culture of foragers in the Late Palaeolithic and the Early Mesolithic (pp. 33-41). Wrocław: University of Wrocław Institute of Archaeology.

Meadows, J., Eriksen, B. V., Zagorska, I., Dreves, A., \& Simpson, J. (2014). Dating Late Palaeolithic harpoons from Lake Lubānas, Latvia. Radiocarbon, 56(2), 581-589.

Mihailović, D., Balaž, I., \& Kapor, D. (2016). Methods in environmental interfaces modelling: Personal insights. Amsterdam: Elsevier.

Møller Hansen, K., \& Buck Pedersen, K. (2006). With or without bones: Late Palaeolithic hunters in south Zealand. In K. Møller Hansen \& K. Buck Pedersen (Eds.), Across the western Baltic. Proceedings of the archaeological conference 'The Prehistory and Early Medieval Period in the Western Baltic' in Vordinborg, South Zealand, Denmark, March 27th-29th 2003 (pp. 93-110). Publikationer 1. Vordinborg: Sydsjællands Museums.

Nowaczyk, B. (1986). Wiek wydm, ich cechy granulometryczne i strukturalne, a schemat cyrkulacji atmosferycznej w Polsce w późnym vistulianie i holocenie. Geografia 28. Poznań: Wydawnictwo Naukowe UAM.

Osipowicz, G., Witas, H., Lisowska-Gaczorek, A., Reitsema, L., Szostek, K., Płoszaj, T., Kuriga, J., Makowiecki, D., Jędrychowska-Dańska, K., \& Cienkosz-Stepańczak, B. (2017). Origin of the ornamented bâton percé from the Gołębiewo site 47 as a trigger of discussion on long-distance exchange among Early Mesolithic communities of central Poland and northern Europe. PLoS ONE, 12(10), e0184560. https://doi.org/10.1371/journal.pone.0184560.

Philippsen, B., Ivanovaitė, L., Makhotka, K., Sauer, F., Riede, F., \& Olsen, J. (2019). Eight new late Pleistocene/early Holocene AMS dates from the southeastern Baltic. Radiocarbon. https://doi. org/10.1017/RDC.2018.153.

Pilarczyk, L. (1958). Wydmy międzyrzecza warciańsko-noteckiego. In Wydmy śródladowe Polski, part I (pp. 87-94). Warszawa: Państwowe Wydawnictwo Naukowe.

Pilarczyk, L. (1976). Międzyrzecze Warciańsko-Noteckie jako pole wydmowe w odniesieniu do powierzchni terasowych i innych. Badania Fizjograficzne nad Polska Zachodnia, Seria A. Geogr. Fiz., 29, 45-80.

Płaza, D. K., Kittel, P., Petera-Zganiacz, J., Dzieduszyńska, D. A., \& Twardy, J. (2015). Late Palaeolithic settlement pattern in palaeogeographical context of the river valleys in the Koło Basin (Central Poland). Quaternary International, 370, 40-54.

Płonka, T. (2003). The portable art of Mesolithic Europe. Acta Universitatis Wratislaviensis 2527. Wrocław: Wydawnictwo Uniwersytetu Wrocławskiego.

Płonka, T. (2012). Kultura symboliczna społeczeństw łowiecko-zbierackich środkowej Europy u schytku paleolitu. Acta Universitatis Wratislaviensis 3430, Studia Archeologiczne 45. Wrocław: Wydawnictwo Uniwersytetu Wrocławskiego.

Płonka, T., \& Kowalski, K. (Eds.). (2017). Rusinowo: The symbolic culture of foragers in the Late Palaeolithic and the Early Mesolithic. Wrocław: University of Wrocław Institute of Archaeology.

Płonka, T., Kowalski, K., Malkiewicz, M., Kuryszko, J., Socha, P., \& Stefaniak, K. (2011). A new ornamented artefact from Poland: Final Palaeolithic symbolism from environmental perspective. Journal of Archaeological Science, 38(3), 723-733. 
R Core Team. (2019). R: A language and environment for statistical computing. Vienna, Austria: R Foundation for Statistical Computing. https://www.R-project.org.

Ralska-Jasiewiczowa, M., Goslar, T., Różański, K., Wacnik, A., Czernik, J., \& Chróst, L. (2003). Very fast environmental changes at the Pleistocene/Holocene boundary, recorded in laminated sediments of Lake Gościąż, Poland. Palaeogeography, Palaeoclimatology, Palaeoecology, 193, 225-247.

Reimer, P. J., Bard, E., Bayliss, A., Warren Beck, J., Blackwell, P. G., Bronk Ramsey, C., et al. (2013). IntCal13 and Marine13 radiocarbon age calibration curves 0-50 000 years cal BP. Radiocarbon, 55(4), 1869-1887.

Rozoy, J.-G. (1978). Les derniers chasseurs, vol. 1-3. Reims: Bulletin de la Société archéologique champenoise, special number.

Rozoy, J.-G. (1989). The revolution of the bowmen in Europe. In C. Bonsall (Ed.), The Mesolithic in Europe. Papers presented at the Third International Symposium, Edinburgh 1985 (pp. 13-28). Edinburgh: John Donald Publishers Ltd.

Rydlewski, J., \& Valde-Vowak, P. (1979). Problem osadnictwa wepoki kamienia w polskich Karpatach Zachodnich w świetle badań na polskiej Orawie i w rejonie prawobrzeżnych dopływów górnej Wisły. Acta Archaeologica Carpathica, 19, 5-36.

Sánchez-Quinto, F., Schroeder, H., Ramirez, O., Ávilla-Marcos, M. C., Pybus, M., Olalde, I., et al. (2012). Genomic affinities of two 7000-year-old Iberian hunter-gatherers. Current Biology, 22, 1494-1499.

Schild, R. (1996a). The north European Plain and eastern Sub-Balticum between 12,700 and 8000 bp. In L. G. Straus, B. V. Eriksen, J. M. Erlandson, \& D. R. Yesner (Eds.), Humans at the end of the Ice Age: The archaeology of the Pleistocene-Holocene transition (pp. 129-157). Interdisciplinary Contributions to Archaeology. New York: Plenum Press.

Schild, R. (1996b). Radiochronology of the early Mesolithic in Poland. In L. Larsson (Ed.), The earliest settlement of Scandinavia and its relationship with neighbouring areas (pp. 285-295). Acta Archaeologica Lundensia 24, series in $8^{\circ}$. Stockholm: Almquist \& Wiksell International.

Schild, R. (1975). Późny paleolit. In W. Hensel (Ed.), Prahistoria ziem polskich (Vol. I, pp. 159-338). Wrocław: Ossolineum.

Schild, R. (2001). Three reasons why it is likely that the early Mesolithic population in Poland was not aboriginal. In B. Ginter (Ed.), Problems of the Stone Age in the Old World. Jubilee book dedicated to Professor Janusz K. Kozłowski (pp. 229-233). Cracow: Institute of Archaeology of the Jagiellonian University.

Schild, R. (2014). Geomorphology, stratigraphy, paleoecology and radiochronology. In R. Schild (Ed.), Catowanie: A Final Paleolithic and Early Mesolithic site on an island in the ancient Vistula channel (pp. 17-57). Vetera et nova. New studies of Archaeological Materials and Data, vol. II. Warsaw: Institute of Archaeology and Ethnology Polish Academy of Sciences.

Schild, R. (2016). Uwagi o stratygrafii stanowiska Wojnowo 2 i chronologii jednostek paleolitycznych, mezolitycznych i paraneolitycznych w Regionie Wojnowa. In M. Kobusiewicz (Ed.), Region Wojnowo: Arkadia łowców i zbieraczy (pp. 461-471). Poznań: Ośrodek Studiów Pradziejowych i Średniowiecznych Instytut Archeologii i Etnologii Polskiej Akademii Nauk.

Schild, R., Królik, H., Tomaszewski, A. J., \& Ciepielewska, E. (2011). Rydno: A Stone Age ochre quarry and socioeconomic center. A century of research. Warsaw: Institute of Archaeology and Ethnology, Polish Academy of Sciences.

Schild, R., \& Więckowska, H. (1961). O tak zwanym przemyśle świdero-tardenuaskim. Archeologia Polski, 6(2), 185-201.

Serwatka, K. (2018). What's your point? Flexible projectile weapon system in the central European Final Palaeolithic. The case of Swiderian points. Journal of Archaeological Science: Reports, 17, 263-278.

Sobkowiak-Tabaka, I. (2011). Spoteczności późnego paleolitu w dorzeczu Odry. Poznań: Instytut Archeologii i Etnologii Polskiej Akademii Nauk.

Sobkowiak-Tabaka, I. (2016). The last Late Glacial hunter-gatherers. In J. Kabaciński (Ed.), The past societies: Polish lands from the first evidence of human presence to the early Middle Ages. Vol. 1: 500,000-5500 BC (pp. 199-227). Warsaw: Institute of Archaeology and Ethnology, Polish Academy of Sciences.

Solon, J., Borzyszkowski, J., Bidłasik, M., Richling, A., Badora, K., Balon, J., et al. (2018). Physicogeographical mesoregions of Poland: Verification and adjustment of boundaries on the basis of contemporary spatial data. Geographia Polonica, 91(2), 143-170. 
Sørensen, M., Lübke, H., \& Groß, D. (2018). The Early Mesolithic in southern Scandinavia and northern Germany. In N. Milner, C. Conneller, \& B. Taylor (Eds.), Star Carr. Volume 1: A persistent place in a changing world (pp. 305-329). York: White Rose University Press.

Starkel, L., Michczyńska, D. J., Krąpiec, M., Margielewski, W., Nalepka, D., \& Pazdur, A. (2013). Progress in the Holocene chrono-climatostratigraphy of Polish territory. Geochronometria, 40(1), $1-21$.

Stoczkowski, W. (1987). The Mesolithic society. In J. K. Kozłowski \& S. K. Kozłowski (Eds.), New in Stone Age archaeology (pp. 85-114). Warsaw: Wydawnictwa Uniwersytetu Warszawskiego (Archaeologia Interregionalis 8).

Street, M. (1991). Bedburg-Königshoven: A preboreal Mesolithic site in the lower Rhineland. In N. Barton, A. J. Roberts, \& D. A. Roe (Eds.), The Late Glacial in north-west Europe: Human adaptation and environmental change at the end of the Pleistocene (pp. 256-270). CBA Research Report 77. London: Council for British Archaeology.

Street, M., Baales, M., Cziesla, E., Hartz, S., Heinen, M., Jöris, O., et al. (2001). Final Paleolithic and Mesolithic research in reunified Germany. Journal of World Prehistory, 15(4), 365-453.

Street, M., Baales, M., \& Weniger, B. (1994). Absolute Chronologie des spätes Paläolithikums im nördlichen Rhineland. Archäologisches Korrespondenzblatt, 24(1), 1-28.

Sulgostowska, Z. (1996). The earliest Mesolithic settlement of north-eastern Poland. In L. Larsson (Ed.), The earliest settlement of Scandinavia and its relationship with neighbouring areas (pp. 297-304). Acta Archaeologica Lundensia 24, series in $8^{\circ}$. Stockholm: Almquist \& Wiksell International.

Sulgostowska, Z. (1997a). The phenomenon of chocolate flint distribution on the North European Plain during the Final Palaeolithic. In R. Schild \& Z. Sulgostowska (Eds.), Man and flint. Proceedings of the VIIth International Flint Symposium, Warszawa-Ostrowiec Świętokrzyski, September 1995 (pp. 313-318). Warsaw: Institute of Archaeology and Ethnology, Polish Academy of Sciences.

Sulgostowska, Z. (1997b). Examples of the application of wood tar during the Mesolithic on Polish territory. In W. Brzeziński, \& W. Piotrowski (Eds.), Proceedings of the First International Symposium on Wood Tar and Pitch (pp. 19-24). Warsaw: State Archaeological Museum.

Sulgostowska, Z. (2005). Kontakty społeczności późno paleolitycznych i mezolitycznych między Odra Dźwina a górnym Dniestrem: Studium dystrybucji wyrobów ze skat krzemionkowych. Warsaw: Instytut Archeologii i Etnologii Polskiej Akademii Nauk.

Sulgostowska, Z. (2008). Szczególna pozycja krzemienia czekoladowego wśród społeczności między Odrą, Dźwiną I Dniestrem u schyłku paleolitu i w późnym mezolicie. In M. Borkowski, J. Libera, B. Sałacińska, \& S. Sałaciński (Eds.), Krzemień czekoladowy w pradziejach: Materiały z konferencji w Orońsku, 08-10.10.2003 (pp. 151-170). Warszawa-Lublin: Studia nad Gospodarką Surowcami Krzemiennymi w Pradziejach 7.

Svoboda, J., Ložek, V., \& Vlček, E. (1996). Hunters between east and west: The Paleolithic of Moravia. New York/London: Plenum Press.

Tanner, V. (1944). Outlines of the geography, life and customs of Newfoundland-Labrador. Acta Geographica, 8, 575-700. Retrieved December 29, 2018, from http://ehrafworldcultures.yale.edu/ document?id=nh06-010.

Terberger, T. (2004). The Younger Dryas: Preboreal transition in northern Germany. Facts and concepts in discussion. In T. Terberger \& B. V. Eriksen (Eds.), Hunters in a changing world: Environment and archaeology of the Pleistocene-Holocene transition (ca. 11000-9000 BC) in northern central Europe. Tagung Symposium, Kongress 5 (pp. 203-222). Verlag Marie Leidorf GmbH.

Terberger, T. (2006). From the first humans to the Mesolithic hunters in the northern German lowlands: Current results and trends. In K. Møller Hansen \& K. Buck Pedersen (Eds.), Across the western Baltic. Proceedings of the Archaeological Conference 'The Prehistory and Early Medieval Period in the Western Baltic' in Vordinborg, South Zealand, Denmark, March 27th-29th 2003 (pp. 23-56). Publikationer 1. Vordinborg: Sydsjællands Museums.

Tolksdorf, J. F., Kaiser, K., Veil, S., Klasen, N., \& Brückner, A. (2009). The Early Mesolithic Haverbeck site, northwest Germany: Evidence for preboreal settlement in the Western and Central European Plain. Journal of Archaeological Science, 36(7), 1466-1476.

Ukkonen, P., Lõugas, L., Zagorska, I., Lukševica, L., Lukševics, E., Daugnora, L., et al. (2006). History of the reindeer (Rangifer tarandus) in the eastern Baltic region and its implications for the origin and immigration routes of the recent northern European wild reindeer populations. Boreas, 35(2), 222-230.

Vang Petersen, P. (2015). Amber pendants, bears and elks. Die Kunde NS, 64 (2013), 219-237. 
Weber, M.-J., Grimm, S. B., \& Baales, M. (2011). Between warm and cold: Impact of the Younger Dryas on human behavior in Central Europe. Quaternary International, 242(2), 277-301.

Weninger, B., Edinborough, K., Bradtmöller, M., Collard, M., Crombé, P., Danzeglocke, U., Holst, D., Jöris, O. Niekus, M., Shennan, S., \& Schulting, R. (2009). A radiocarbon database for the Mesolithic and Early Neolithic in northwest Europe. In Chronology and evolution within the Mesolithic of north-west Europe. Proceedings of an international meeting, Brussels, May 30th-June 1 st 2007 (pp. 143-176). Newcastle-upon-Tyne: Cambridge Scholars Publishing.

Whallon, R. (2006). Social networks and information: Non-'utilitarian' mobility among hunters-gatherers. Journal of Anthropological Archaeology, 25(2), 259-270.

Więckowska, H. (1975). Społeczności łowiecko-rybackie wczesnego holocenu. In W. Hensel (Ed.), Prahistoria ziem polskich (Vol. I, pp. 339-438). Wrocław: Ossolineum.

Zagorska, I. (2006). The earliest antler and bone harpoons from the East Baltic. Archaeologia Baltica, 7(2005), 178-186.

Zander, A. (2016). Heek-Nienborg and Werl-Büderich: The transition from Palaeolithic to Mesolithic in Westphalia. Archäologische Informationen, 39, 285-291.

Zeeberg, J. (1998). The European sand belt in eastern Europe-And comparison of Late Glacial dune orientation with GCM simulation results. Boreas, 27, 127-139.

Zotz, L. F. (1934). Culture groups of the Tardenoisien in Central Europe. American Anthropologist, $36(2), 242-265$.

Publisher's Note Springer Nature remains neutral with regard to jurisdictional claims in published maps and institutional affiliations. 